\title{
Palladium(II) Ion Mediated Disulfide/Thiolate Interconversion: Predicting The Disulfide Group State From The First Principles
}

\author{
Alexander I. Petrov*,1, Vsevolod D. Dergachev ${ }^{2,3}$ \\ ${ }^{1}$ Institute of Chemistry and Chemical Technology SB RAS, Federal Research Center \\ "Krasnoyarsk Science Center SB RAS", Krasnoyarsk, Russian Federation \\ 2 Department of Chemistry, University of Nevada, Reno, USA \\ ${ }^{3}$ Institute of Informatics and Telecommunications, Siberian State Aerospace University, \\ Krasnoyarsk, Russian Federation
}

*Corresponding author e-mail: sfupetrov@gmail.com

Table S1. The absolute electronic energies (Gas), the zero-point energies with thermal corrections $\left(\mathrm{G}^{\mathrm{T}}\right)$, the solvation free energies (SMD), and solvation free energy (solv) at the DEF2TZVPD/PBE0-D3/SMD Level of Theory

\begin{tabular}{|c|c|c|c|c|}
\hline Name & Gas, a.u. & $\mathrm{G}^{\mathrm{T}}$, a.u. & SMD, a.u, & solv., a.u. \\
\hline $\mathrm{Cl}^{-}$ & $-460,1293426$ & & $-460,232646$ & $-0,103303$ \\
\hline$\left[\mathrm{PdCl}_{4}\right]^{2-}$ & $-1968,3569938$ & 0,004112 & $-1968,630004$ & $-0,273011$ \\
\hline $\mathrm{H}_{2} \mathrm{Cyst}^{2+}$ & $-1065,7234200$ & 0,200595 & $-1066,01030$ & $-0,28688$ \\
\hline $\mathrm{H}_{4} \mathrm{CysS}^{2+}$ & $-1442,6452409$ & 0,229708 & $-1442,919933$ & $-0,274692$ \\
\hline$\left[\mathrm{Pd}_{2} \mathrm{Cl}_{6}\left(\mathrm{~N}, \mathrm{~N}^{\prime}-\mathrm{Cyst}\right)\right]^{2-}$ & $-4081,7191971$ & 0,184720 & $-4081,930031$ & $-0,210834$ \\
\hline$\left[\mathrm{Pd}_{2} \mathrm{Cl}_{6}\left(\mathrm{~S}, \mathrm{~S}^{\prime}-\mathrm{H}_{2} \mathrm{Cyst}\right)\right]^{0}$ & $-4082,7302182$ & 0,208536 & $-4082,816088$ & $-0,085870$ \\
\hline$\left[\mathrm{Pd}_{2} \mathrm{Cl}_{4}(\mathrm{~S}, \mathrm{~N}-\mathrm{Cyst})\right]$ & $-3161,3959174$ & 0,184967 & $-3161,436962$ & $-0,041044$ \\
\hline $\begin{array}{c}{\left[\mathrm{Pd}_{4} \mathrm{Cl}_{12}\left(\mathrm{~S}, \mathrm{~S}^{\prime}, \mathrm{N}, \mathrm{N}^{\prime}-\right.\right.} \\
\mathrm{HCyst})]^{4-}\end{array}$ & $-7098,1112071$ & 0,193106 & $-7098,741022$ & $-0,629815$ \\
\hline $\begin{array}{c}{\left[\mathrm{Pd}_{3} \mathrm{Cl}_{9}\left(\mathrm{~S}, \mathrm{~S}^{\prime}, \mathrm{N}-\right.\right.} \\
\mathrm{HCyst})]^{2-}\end{array}$ & $-5590,5402954$ & 0,201405 & $-5590,782383$ & $-0,242088$ \\
\hline$\left[\mathrm{PdCl}_{2}(\mathrm{~S}, \mathrm{~N}-\mathrm{HCyst})\right]^{+}$ & $-2113,6204245$ & 0,191489 & $-2113,728945$ & $-0,108525$ \\
\hline$\left[\mathrm{PdCl}_{3}\left(\mathrm{~S}-\mathrm{H}_{2} \mathrm{Cyst}\right)\right]^{+}$ & $-2574,2920939$ & 0,204008 & $-2574,409783$ & $-0,117689$ \\
\hline$\left[\mathrm{PdCl}\left(\mathrm{S}, \mathrm{N}, \mathrm{N}^{\prime}-\mathrm{Cyst}\right)\right]^{+}$ & $-1652,9488788$ & 0,179801 & $-1653,03235$ & $-0,083471$ \\
\hline$\left[\mathrm{Pd}_{3} \mathrm{Cl}_{9}\left(\mathrm{~S}, \mathrm{~N}, \mathrm{~N}^{\prime}-\mathrm{Cyst}\right)\right]^{3-}$ & $-5589,9492369$ & 0,190877 & $-5590,337924$ & $-0,3886870$ \\
\hline$\left[\mathrm{Pd}_{2} \mathrm{Cl}_{6}(\mathrm{~S}, \mathrm{~N}-\mathrm{HCyst})\right]^{-}$ & $-4082,2584760$ & 0,196856 & $-4082,379759$ & $-0,121283$ \\
\hline $\begin{array}{c}{\left[\mathrm{Pd}_{2} \mathrm{Cl}_{6}\left(\mathrm{~N}, \mathrm{~N}^{\prime}-\right.\right.} \\
\left.\left.\mathrm{H}_{2} \mathrm{CysS}\right)\right]^{2-}\end{array}$ & $-4458,6414047$ & 0,215073 & $-4458,857342$ & $-0,215937$ \\
\hline$\left[\mathrm{Pd}_{2} \mathrm{Cl}_{4}\left(\mathrm{~S}, \mathrm{~N}-\mathrm{H}_{2} \mathrm{CysS}\right)\right]$ & $-3538,3192608$ & 0,214122 & $-3538,360955$ & $-0,041694$ \\
\hline $\begin{array}{c}{[\mathrm{PdCl}(\mathrm{S}, \mathrm{N}, \mathrm{O}-} \\
\left.\left.\mathrm{H}_{3} \mathrm{CysS}\right)\right]^{2+} \\
\end{array}$ & $-2030,0630431$ & 0,221854 & $-2030,355566$ & $-0,292523$ \\
\hline $\begin{array}{c}\mathrm{PdCl}\left(\mathrm{S}, \mathrm{N}, \mathrm{N}^{\prime}-\right. \\
\left.\left.\mathrm{H}_{2} \mathrm{CysS}\right)\right]^{+}\end{array}$ & $-2029,8702914$ & 0,210033 & $-2029,956867$ & $-0,086576$ \\
\hline $\begin{array}{c}{\left[\mathrm{Pd}_{3} \mathrm{Cl}_{9}\left(\mathrm{~S}, \mathrm{~S}^{\prime}, \mathrm{N}-\right.\right.} \\
\left.\left.\mathrm{H}_{3} \mathrm{CysS}\right)\right]^{2-} \\
\end{array}$ & $-5967,4654137$ & 0,229809 & $-5967,701533$ & $-0,236119$ \\
\hline$\left[\mathrm{PdCl}_{3}\left(\mathrm{~S}-\mathrm{H}_{4} \mathrm{CysS}\right)\right]^{+}$ & $-2951,2072774$ & 0,233950 & $-2951,324943$ & $-0,117666$ \\
\hline$\left[\mathrm{PdCl}_{3}\left(\mathrm{~N}-\mathrm{H}_{3} \mathrm{CysS}\right)\right]^{0}$ & $-2950,8183061$ & 0,222220 & $-2950,892555$ & $-0,074249$ \\
\hline
\end{tabular}




\begin{tabular}{|c|c|c|c|c|}
\hline $\begin{array}{c}{\left[\mathrm{Pd}_{3} \mathrm{Cl}_{9}\left(\mathrm{~S}, \mathrm{~N}, \mathrm{~N}^{\prime}-\right.\right.} \\
\left.\left.\mathrm{H}_{2} \mathrm{CysS}\right)\right]^{3-}\end{array}$ & $-5966,8615566$ & 0,219171 & $-5967,254398$ & $-0,392842$ \\
\hline $\begin{array}{c}{\left[\mathrm{Pd}_{4} \mathrm{Cl}_{12}\left(\mathrm{~S}, \mathrm{~S}^{\prime}, \mathrm{N}, \mathrm{N}^{\prime}-\right.\right.} \\
\left.\left.\mathrm{H}_{2} \mathrm{CysS}\right)\right]^{4-}\end{array}$ & $-7475,0269993$ & 0,224799 & $-7475,664543$ & $-0,637544$ \\
\hline$\left[\mathrm{PdCl}_{2}\left(\mathrm{~S}, \mathrm{~N}-\mathrm{H}_{3} \mathrm{CysS}\right)\right]^{+}$ & $-2490,5192638$ & 0,222368 & $-2490,643254$ & $-0,12399$ \\
\hline$\left[\mathrm{PdCl}_{2}\left(\mathrm{~S}, \mathrm{O}-\mathrm{H}_{4} \mathrm{CysS}\right)\right]^{2+}$ & $-2490,7391270$ & 0,234414 & $-2491,039642$ & $-0,300515$ \\
\hline$\left[\mathrm{PdCl}_{2}\left(\mathrm{~N}, \mathrm{O}-\mathrm{H}_{3} \mathrm{CysS}\right)\right]^{+}$ & $-2490,4751585$ & 0,221624 & $-2490,620988$ & $-0,145829$ \\
\hline$\left[\mathrm{Pd}_{2} \mathrm{Cl}_{6}\left(\mathrm{~S}, \mathrm{~S}^{\prime}-\mathrm{H}_{4} \mathrm{CysS}\right)\right]^{0}$ & $-4459,6575632$ & 0,239400 & $-4459,732652$ & $-0,075089$ \\
\hline$\left[\mathrm{Pd}_{2} \mathrm{Cl}_{6}\left(\mathrm{~S}, \mathrm{~N}-\mathrm{H}_{3} \mathrm{CysS}\right)\right]^{-}$ & $-4459,1976396$ & 0,228087 & $-4459,30164$ & $-0,104001$ \\
\hline $\mathrm{H}_{2} \mathrm{O}$ & $-76,3808133$ & 0,021421 & $-76,391645$ & $-0,010832$ \\
\hline $\mathrm{H}_{4} \mathrm{HCysS}^{2+}$ & $-1521,2266650$ & 0,286848 & $-1521,490557$ & $-0,263892$ \\
\hline $\mathrm{H}_{2}$ DTDPA & $-1331,3839008$ & 0,167494 & $-1331,400287$ & $-0,016387$ \\
\hline$\left[\mathrm{PdCl}_{3}\left(\mathrm{~S}-\mathrm{HCysteO}{ }_{2} \mathrm{H}\right)\right]$ & $-2192,3121352$ & 0,122048 & $-2192,363524$ & $-0,0513886$ \\
\hline$\left[\mathrm{PdCl}_{3}\left(\mathrm{~S}-\mathrm{H}_{2} \mathrm{CysO}_{2} \mathrm{H}\right)\right]$ & $-2380,7525558$ & 0,135589 & $-2380,820442$ & $-0,067886$ \\
\hline$\left[\mathrm{PdCl}_{3}\left(\mathrm{~S}-\mathrm{H}_{2} \mathrm{Cys}\right)\right]^{-}$ & $-2229,9206171$ & 0,117294 & $-2230,028788$ & $-0,108171$ \\
\hline$\left[\mathrm{PdCl}_{3}\left(\mathrm{~S}-\mathrm{H}_{2} \mathrm{Cyste}\right)\right]$ & $-2041,4553237$ & 0,101499 & $-2041,566219$ & $-0,110896$ \\
\hline$\left[\mathrm{PdCl}_{3}\left(\mathrm{~S}-\mathrm{H}_{3} \mathrm{CSA}\right)\right]$ & $-2420,0515538$ & 0,166509 & $-2420,107607$ & $-0,0560532$ \\
\hline$\left[\mathrm{PdCl}_{3}\left(\mathrm{~S}-\mathrm{H}_{2} \mathrm{HCys}\right)\right]^{-}$ & $-2269,2031707$ & 0,143973 & $-2269,308340$ & $-0,105169$ \\
\hline$\left[\mathrm{PdCl}_{3}\left(\mathrm{~S}-\mathrm{H}_{2} \mathrm{SPA}\right)\right]^{2-}$ & $-2324,9787263$ & 0,106919 & $-2325,05668$ & $-0,077954$ \\
\hline$\left[\mathrm{PdCl}_{3}\left(\mathrm{~S}-\mathrm{H}_{2} \mathrm{MPA}\right)\right]^{-}$ & $-2173,9813371$ & 0,086167 & $-2174,242173$ & $-0,260836$ \\
\hline $\mathrm{H}_{2} \mathrm{MPA}$ & $-666,2807821$ & 0,091093 & $-666,289743$ & $-0,008961$ \\
\hline $\mathrm{H}_{3} \mathrm{Cys}^{+}$ & $-721,9568802$ & 0,122965 & $-722,056985$ & $-0,100105$ \\
\hline $\mathrm{H}_{3} \mathrm{HCys}^{+}$ & $-761,2387895$ & 0,15218 & $-761,337354$ & $-0,098565$ \\
\hline $\mathrm{H}_{2}$ Cyste $^{+}$ & $-533,4927309$ & 0,107558 & $-533,594266$ & $-0,101535$ \\
\hline $\mathrm{H}_{2} \mathrm{SPA}$ & $-816,6259301$ & 0,101256 & $-816,64498$ & $-0,01905$ \\
\hline $\mathrm{H}_{2} \mathrm{CysO}_{2} \mathrm{H}^{+}$ & $-872,3084625$ & 0,132770 & $-872,408959$ & $-0,100497$ \\
\hline $\mathrm{H}_{3} \mathrm{HCSA}^{+}$ & $-911,5993878$ & 0,162360 & $-911,695465$ & $-0,096077$ \\
\hline $\mathrm{HCysteO}_{2} \mathrm{H}^{+}$ & $-683,8473060$ & 0,117553 & $-683,952011$ & $-0,104705$ \\
\hline $\begin{array}{c}{\left[\mathrm{Pd}_{2} \mathrm{Cl}_{6}\left(\mathrm{~S}, \mathrm{~S}^{\prime}-\right.\right.} \\
\left.\left.\mathrm{H}_{4} \mathrm{hCysS}\right)\right]^{0}\end{array}$ & $-4538,221714$ & 0,297874 & $-4538,303406$ & $-0,0816918$ \\
\hline$\left[\mathrm{PdCl}_{3}\left(\mathrm{~S}-\mathrm{H}_{4} \mathrm{hCysS}\right)\right]^{+}$ & $-3031,530648$ & 0,297575 & $-3031,634050$ & $-0,1034021$ \\
\hline$\left[\mathrm{PdCl}_{3}\left(\mathrm{~S}-\mathrm{H}_{2} \mathrm{DTPDA}\right)\right]^{-}$ & $-2839,718055$ & 0,172356 & $-2839,807223$ & $-0,089168$ \\
\hline $\begin{array}{l}{\left[\mathrm{Pd}_{2} \mathrm{Cl}_{6}\left(\mathrm{~S}, \mathrm{~S}^{\prime}-\right.\right.} \\
\left.\left.\mathrm{H}_{2} \mathrm{DTPDA}\right)\right]^{2-}\end{array}$ & $-4347,98025$ & 0,176482 & $-4348,203709$ & $-0,223459$ \\
\hline
\end{tabular}


Table S2. The absolute electronic energies (Gas), the zero-point energies with thermal corrections $\left(\mathrm{G}^{\mathrm{T}}\right)$, the solvation free energies (SMD), and solvation free energy (solv) at the DEF2TZVPD/TPSSh-D3/SMD Level of Theory

\begin{tabular}{|c|c|c|c|c|}
\hline Name & Gas, a.u. & $\mathrm{G}^{\mathrm{T}}$, a.u. & SMD, a.u, & solv., a.u. \\
\hline $\mathrm{Cl}^{-}$ & $-460,2959171$ & & $-460,3991475$ & $-0,103231$ \\
\hline$\left[\mathrm{PdCl}_{4}\right]^{2-}$ & $-1968,993088$ & 0,004112 & $-1969,262856$ & $-0,269767$ \\
\hline $\mathrm{H}_{2} \mathrm{Cyst}^{2+}$ & $-1066,377270$ & 0,200595 & $-1066,663134$ & $-0,285864$ \\
\hline $\mathrm{H}_{4} \mathrm{CysS}^{2+}$ & $-1443,685748$ & 0,229708 & $-1443,959545$ & $-0,273802$ \\
\hline$\left[\mathrm{Pd}_{2} \mathrm{Cl}_{6}\left(\mathrm{~N}, \mathrm{~N}^{\prime}-\mathrm{Cyst}\right)\right]^{2-}$ & $-4083,003762$ & 0,184720 & $-4083,604700$ & $-0,209600$ \\
\hline$\left[\mathrm{Pd}_{2} \mathrm{Cl}_{6}\left(\mathrm{~S}, \mathrm{~S}^{\prime}-\mathrm{H}_{2} \mathrm{Cyst}\right)\right]^{0}$ & $-4084,422092$ & 0,208536 & $-4084,502290$ & $-0,080197$ \\
\hline$\left[\mathrm{Pd}_{2} \mathrm{Cl}_{4}(\mathrm{~S}, \mathrm{~N}-\mathrm{Cyst})\right]$ & $-3162,733954$ & 0,184967 & $-3162,772828$ & $-0,038874$ \\
\hline $\begin{array}{c}{\left[\mathrm{Pd}_{4} \mathrm{Cl}_{12}\left(\mathrm{~S}, \mathrm{~S}^{\prime}, \mathrm{N}, \mathrm{N}^{\prime}-\right.\right.} \\
\mathrm{HCyst})]^{4-}\end{array}$ & $-7100,782905$ & 0,193106 & $-7101,409886$ & $-0,626980$ \\
\hline $\begin{array}{c}{\left[\mathrm{Pd}_{3} \mathrm{Cl}_{9}\left(\mathrm{~S}, \mathrm{~S}^{\prime}, \mathrm{N}-\right.\right.} \\
\mathrm{HCyst})]^{2-}\end{array}$ & $-5592,722392$ & 0,201405 & $-5592,960233$ & $-0,237841$ \\
\hline$\left[\mathrm{PdCl}_{2}(\mathrm{~S}, \mathrm{~N}-\mathrm{HCyst})\right]^{+}$ & $-2114,634973$ & 0,191489 & $-2114,741669$ & $-0,106695$ \\
\hline$\left[\mathrm{PdCl}_{3}\left(\mathrm{~S}-\mathrm{H}_{2} \mathrm{Cyst}\right)\right]^{+}$ & $-2575,483437$ & 0,204008 & $-2575,598992$ & $-0,115555$ \\
\hline$\left[\mathrm{PdCl}\left(\mathrm{S}, \mathrm{N}, \mathrm{N}^{\prime}-\mathrm{Cyst}\right)\right]^{+}$ & $-1653,7897916$ & 0,179801 & $-1653,7876234$ & 0,0021682 \\
\hline$\left[\mathrm{Pd}_{3} \mathrm{Cl}_{9}\left(\mathrm{~S}, \mathrm{~N}, \mathrm{~N}^{\prime}-\mathrm{Cyst}\right)\right]^{3-}$ & $-4083,9345449$ & 0,190877 & $-4084,0566728$ & $-0,122128$ \\
\hline$\left[\mathrm{Pd}_{2} \mathrm{Cl}_{6}(\mathrm{~S}, \mathrm{~N}-\mathrm{HCyst})\right]^{-}$ & $-4083,9414765$ & 0,196856 & $-4084,0596063$ & $-0,118130$ \\
\hline $\begin{array}{c}{\left[\mathrm{Pd}_{2} \mathrm{Cl}_{6}\left(\mathrm{~N}, \mathrm{~N}^{\prime}-\right.\right.} \\
\left.\left.\mathrm{H}_{2} \mathrm{CysS}\right)\right]^{2-}\end{array}$ & $-4460,6105438$ & 0,215073 & $-4460,8264485$ & $-0,215905$ \\
\hline$\left[\mathrm{Pd}_{2} \mathrm{Cl}_{4}\left(\mathrm{~S}, \mathrm{~N}^{\prime}-\mathrm{H}_{2} \mathrm{CysS}\right)\right]$ & $-3539,9599245$ & 0,214122 & $-3540,0008067$ & $-0,040882$ \\
\hline $\begin{array}{c}{\left[\mathrm{PdCl}\left(\mathrm{S}, \mathrm{N}, \mathrm{O}^{\prime}-\right.\right.} \\
\left.\left.\mathrm{H}_{3} \mathrm{CysS}\right)\right]^{2+}\end{array}$ & $-2031,2383936$ & 0,221854 & $-2031,5299095$ & $-0,291516$ \\
\hline $\begin{array}{c}{\left[\mathrm{PdCl}\left(\mathrm{S}, \mathrm{N}, \mathrm{N}^{\prime}-\right.\right.} \\
\left.\left.\mathrm{H}_{2} \mathrm{CysS}\right)\right]^{+}\end{array}$ & $-2031,0424303$ & 0,210033 & $-2031,1289210$ & $-0,086491$ \\
\hline $\begin{array}{c}{\left[\mathrm{Pd}_{3} \mathrm{Cl}_{9}\left(\mathrm{~S}, \mathrm{~S}^{\prime}, \mathrm{N}-\right.\right.} \\
\left.\left.\mathrm{H}_{3} \mathrm{CysS}\right)\right]^{2-}\end{array}$ & $-5969,9101956$ & 0,229809 & $-5970,1442057$ & $-0,234010$ \\
\hline$\left[\mathrm{PdCl}_{3}\left(\mathrm{~S}-\mathrm{H}_{4} \mathrm{CysS}\right)\right]^{+}$ & $-2952,7177826$ & 0,233950 & $-2952,8344086$ & $-0,116626$ \\
\hline$\left[\mathrm{PdCl}_{3}\left(\mathrm{~N}-\mathrm{H}_{3} \mathrm{CysS}\right)\right]^{0}$ & $-2952,3252122$ & 0,222220 & $-2952,3979704$ & $-0,072758$ \\
\hline $\begin{array}{c}{\left[\mathrm{Pd}_{3} \mathrm{Cl}_{9}\left(\mathrm{~S}, \mathrm{~N}, \mathrm{~N}^{\prime}-\right.\right.} \\
\left.\left.\mathrm{H}_{2} \mathrm{CysS}\right)\right]^{3-}\end{array}$ & $-5969,2995818$ & 0,219171 & $-5969,6925232$ & $-0,392941$ \\
\hline $\begin{array}{c}{\left[\mathrm{Pd}_{4} \mathrm{Cl}_{12}\left(\mathrm{~S}, \mathrm{~S}^{\prime}, \mathrm{N}, \mathrm{N}^{\prime}-\right.\right.} \\
\left.\left.\mathrm{H}_{2} \mathrm{CysS}\right)\right]^{4-}\end{array}$ & $-7477,9343127$ & 0,224799 & $-7478,5714388$ & $-0,637126$ \\
\hline$\left[\mathrm{PdCl}_{2}\left(\mathrm{~S}, \mathrm{~N}-\mathrm{H}_{3} \mathrm{CysS}\right)\right]^{+}$ & $-2491,8596174$ & 0,222368 & $-2491,9825657$ & $-0,122948$ \\
\hline$\left[\mathrm{PdCl}_{2}\left(\mathrm{~S}, \mathrm{O}-\mathrm{H}_{4} \mathrm{CysS}\right)\right]^{2+}$ & $-2492,0836750$ & 0,234414 & $-2492,3827283$ & $-0,299053$ \\
\hline$\left[\mathrm{PdCl}_{2}\left(\mathrm{~N}, \mathrm{O}-\mathrm{H}_{3} \mathrm{CysS}\right)\right]^{+}$ & $-2491,8148625$ & 0,221624 & $-2491,9602974$ & $-0,145435$ \\
\hline$\left[\mathrm{Pd}_{2} \mathrm{Cl}_{6}\left(\mathrm{~S}, \mathrm{~S}^{\prime}-\mathrm{H}_{4} \mathrm{CysS}\right)\right]^{0}$ & $-4461,6363822$ & 0,239400 & $-4461,7098073$ & $-0,070929$ \\
\hline$\left[\mathrm{Pd}_{2} \mathrm{Cl}_{6}\left(\mathrm{~S}, \mathrm{~N}-\mathrm{H}_{3} \mathrm{CysS}\right)\right]^{-}$ & $-4461,1712606$ & 0,228087 & $-4461,2748156$ & $-0,103555$ \\
\hline $\mathrm{H}_{2} \mathrm{O}$ & $-76,4593389$ & 0,021421 & $-76,4698866$ & $-0,010548$ \\
\hline
\end{tabular}




\begin{tabular}{|c|c|c|c|c|}
\hline $\mathrm{H}_{4} \mathrm{HCysS}^{2+}$ & $-1522,3721048$ & 0,286848 & $-1522,6352206$ & $-0,263116$ \\
\hline $\mathrm{H}_{2}$ DTDPA & $-1332,2994460$ & 0,167494 & $-1332,3155311$ & $-0,016085$ \\
\hline$\left[\mathrm{PdCl}_{3}\left(\mathrm{~S}-\mathrm{HCysteO}_{2} \mathrm{H}\right)\right]$ & $-2193,259459$ & 0,122048 & $-2193,310147$ & $-0,050688$ \\
\hline$\left[\mathrm{PdCl}_{3}\left(\mathrm{~S}-\mathrm{H}_{2} \mathrm{CysO}_{2} \mathrm{H}\right)\right]$ & $-2381,892917$ & 0,135589 & $-2381,960173$ & $-0,067255$ \\
\hline$\left[\mathrm{PdCl}_{3}\left(\mathrm{~S}-\mathrm{H}_{2} \mathrm{Cys}\right)\right]^{-}$ & $-2230,907966$ & 0,117294 & $-2231,015935$ & $-0,107969$ \\
\hline$\left[\mathrm{PdCl}_{3}\left(\mathrm{~S}-\mathrm{H}_{2} \mathrm{Cyste}\right)\right]$ & $-2042,250094$ & 0,101499 & $-2042,360242$ & $-0,110148$ \\
\hline$\left[\mathrm{PdCl}_{3}\left(\mathrm{~S}-\mathrm{H}_{3} \mathrm{CSA}\right)\right]$ & $-2421,244554$ & 0,166509 & $-2421,300360$ & $-0,055807$ \\
\hline$\left[\mathrm{PdCl}_{3}\left(\mathrm{~S}-\mathrm{H}_{2} \mathrm{HCys}\right)\right]^{-}$ & $-2270,243057$ & 0,143973 & $-2270,348029$ & $-0,104972$ \\
\hline$\left[\mathrm{PdCl}_{3}\left(\mathrm{~S}-\mathrm{H}_{2} \mathrm{SPA}\right)\right]^{2-}$ & $-2326,057317$ & 0,106919 & $-2326,134516$ & $-0,077199$ \\
\hline$\left[\mathrm{PdCl}_{3}\left(\mathrm{~S}-\mathrm{H}_{2} \mathrm{MPA}\right)\right]^{-}$ & $-2174,907036$ & 0,086167 & $-2175,167266$ & $-0,260231$ \\
\hline $\mathrm{H}_{2} \mathrm{MPA}$ & $-666,768646$ & 0,091093 & $-666,777178$ & $-0,008532$ \\
\hline $\mathrm{H}_{3} \mathrm{Cys}^{+}$ & $-722,506759$ & 0,122965 & $-722,606833$ & $-0,100074$ \\
\hline $\mathrm{H}_{3} \mathrm{HCys}^{+}$ & $-761,838117$ & 0,15218 & $-761,935485$ & $-0,097368$ \\
\hline $\mathrm{H}_{2}$ Cyste $^{+}$ & $-533,839369$ & 0,107558 & $-533,940008$ & $-0,100639$ \\
\hline $\mathrm{H}_{2} \mathrm{SPA}$ & $-817,238035$ & 0,101256 & $-817,256429$ & $-0,018394$ \\
\hline $\mathrm{H}_{2} \mathrm{CysO}_{2} \mathrm{H}^{+}$ & $-872,983977$ & 0,132770 & $-873,085832$ & $-0,101855$ \\
\hline $\mathrm{H}_{3} \mathrm{HCSA}^{+}$ & $-912,320524$ & 0,162360 & $-912,415702$ & $-0,095178$ \\
\hline $\mathrm{HCysteO}_{2} \mathrm{H}^{+}$ & $-684,327759$ & 0,117553 & $-684,431972$ & $-0,104213$ \\
\hline $\begin{array}{c}\mathrm{Pd}_{2} \mathrm{Cl}_{6}\left(\mathrm{~S}, \mathrm{~S}^{\prime}-\right. \\
\left.\left.\mathrm{H}_{4} \mathrm{hCysS}\right)\right]^{0}\end{array}$ & $-4540,3063299$ & 0,2978740 & $-4540,3865585$ & $-0,080229$ \\
\hline$\left[\mathrm{PdCl}_{3}\left(\mathrm{~S}-\mathrm{H}_{4} \mathrm{hCysS}\right)\right]^{+}$ & $-3031,4097947$ & 0,2975750 & $-3031,5146877$ & $-0,104893$ \\
\hline$\left[\mathrm{PdCl}_{3}\left(\mathrm{~S}-\mathrm{H}_{2} \mathrm{DTPDA}\right)\right]^{-}$ & $-2841,102351$ & 0,172356 & $-2841,190287$ & $-0,087936$ \\
\hline $\begin{array}{c}{\left[\mathrm{Pd}_{2} \mathrm{Cl}_{6}\left(\mathrm{~S}, \mathrm{~S}^{\prime}-\right.\right.} \\
\left.\left.\mathrm{H}_{2} \mathrm{DTPDA}\right)\right]^{2-} \\
\end{array}$ & $-4349,834541$ & 0,176482 & $-4350,056373$ & $-0,221831$ \\
\hline
\end{tabular}



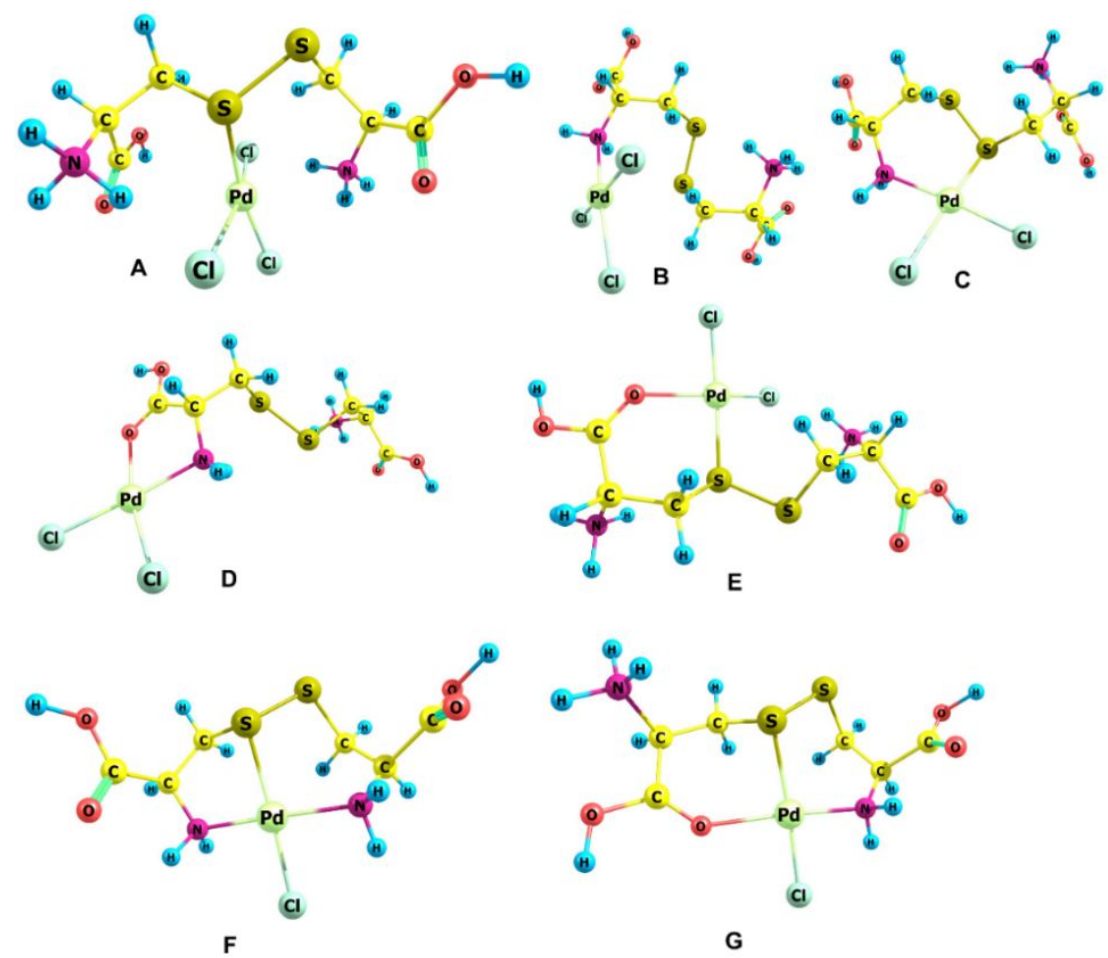

Figure S1. PBE0/Def2-SVP-D3 structures of L-cystine monocomplexes: $\left[\mathrm{PdCl}_{3}\left(\mathrm{~S}-\mathrm{H}_{4} \mathrm{CysS}\right)\right]^{+}$ (A), $\left[\mathrm{PdCl}_{3}\left(\mathrm{~N}-\mathrm{H}_{3} \mathrm{CysS}\right)\right]^{0}$ (B), $\left[\mathrm{PdCl}_{2}\left(\mathrm{~S}, \mathrm{~N}-\mathrm{H}_{3} \mathrm{CysS}\right)\right]^{+}(\mathbf{C}),\left[\mathrm{PdCl}_{2}\left(\mathrm{~N}, \mathrm{O}-\mathrm{H}_{3} \mathrm{CysS}\right)\right]^{+}(\mathbf{D})$, $\left[\mathrm{PdCl}_{2}\left(\mathrm{~S}, \mathrm{O}-\mathrm{H}_{4} \mathrm{CysS}\right)\right]^{2+}(\mathbf{E}),\left[\mathrm{PdCl}\left(\mathrm{S}, \mathrm{N}, \mathrm{N}^{\prime}-\mathrm{H}_{2} \mathrm{CysS}\right)\right]^{+}(\mathbf{F}),\left[\mathrm{PdCl}\left(\mathrm{S}, \mathrm{N}, \mathrm{O}-\mathrm{H}_{3} \mathrm{CysS}\right)\right]^{2+}(\mathbf{G})$
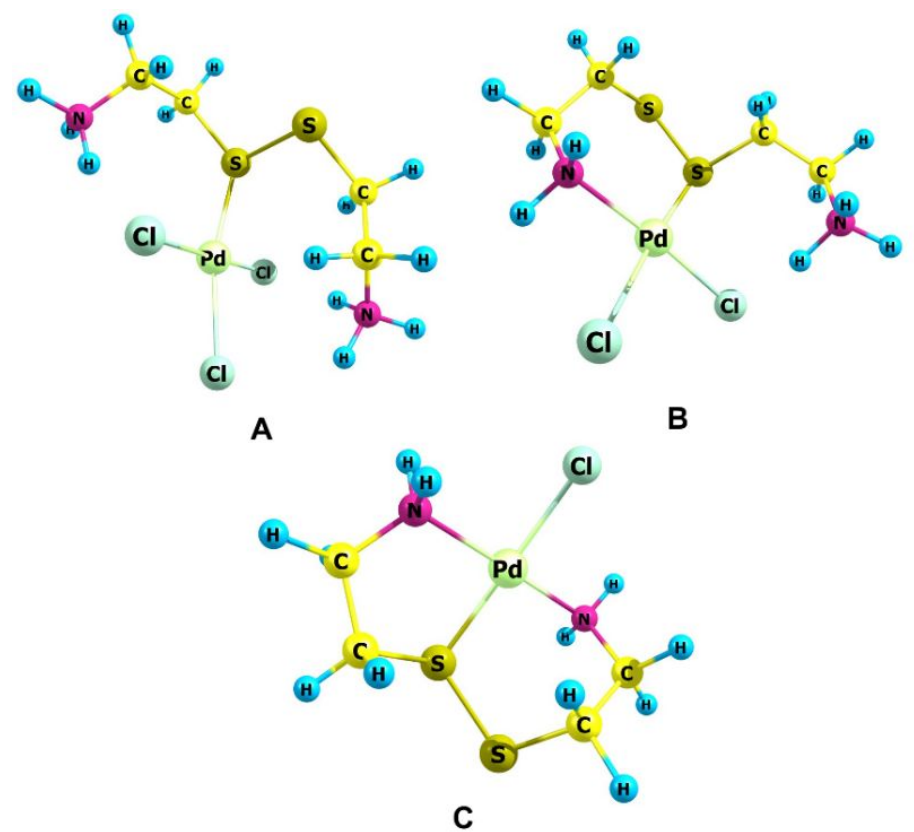

Figure S2. PBE0/Def2-SVP-D3 structures of cystamine monocomplexes: $\left[\mathrm{PdCl}_{3}\left(\mathrm{~S}-\mathrm{H}_{2} \mathrm{Cyst}\right)\right]^{+}$ (A), $\left[\mathrm{PdCl}_{2}(\mathrm{~S}, \mathrm{~N}-\mathrm{HCyst})\right]^{+}(\mathbf{B}),\left[\mathrm{PdCl}\left(\mathrm{S}, \mathrm{N}, \mathrm{N}^{\prime}-\mathrm{Cyst}\right)\right]^{+}(\mathbf{C})$ 

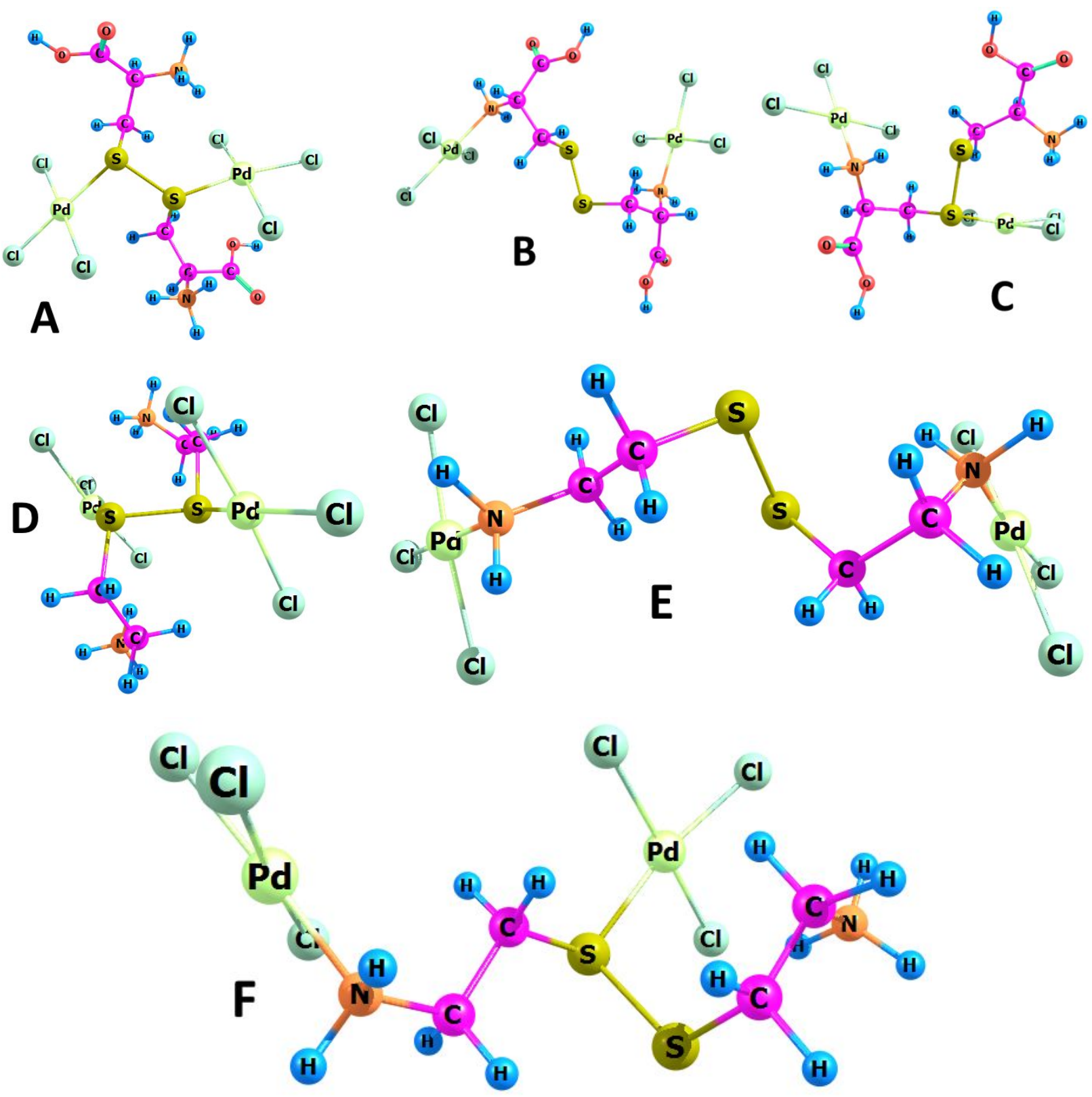

Figure S3. PBE0/Def2-SVP-D3 structures of binuclear complexes of L-cystine $(\mathbf{A}, \mathbf{B}, \mathbf{C})$ and cystamine (D, E, F): $\left[\mathrm{Pd}_{2} \mathrm{Cl}_{6}\left(\mathrm{~S}, \mathrm{~S}^{\prime}-\mathrm{H}_{4} \mathrm{CysS}\right)\right]^{0}(\mathbf{A}),\left[\mathrm{Pd}_{2} \mathrm{Cl} 6{ }_{6}\left(\mathrm{~N}, \mathrm{~N}^{\prime}-\mathrm{H}_{2} \mathrm{CysS}\right)\right]^{2-}(\mathbf{B}),{ }_{\mathrm{Pd}_{2}} \mathrm{Cl}_{6}(\mathrm{~S}, \mathrm{~N}-$ $\left.\left.\mathrm{H}_{3} \mathrm{CysS}\right)\right]^{-}(\mathbf{C}),\left[\mathrm{Pd}_{2} \mathrm{Cl}_{6}\left(\mathrm{~S}, \mathrm{~S}^{\prime}-\mathrm{H}_{2} \mathrm{Cyst}\right)\right]^{0}(\mathbf{D}),\left[\mathrm{Pd}_{2} \mathrm{Cl}_{6}\left(\mathrm{~N}, \mathrm{~N}^{\prime}-\mathrm{Cyst}\right)\right]^{2-}(\mathbf{E}),\left[\mathrm{Pd}_{2} \mathrm{Cl}_{6}(\mathrm{~S}, \mathrm{~N}-\mathrm{HCyst})\right]^{-}(\mathbf{F})$. 


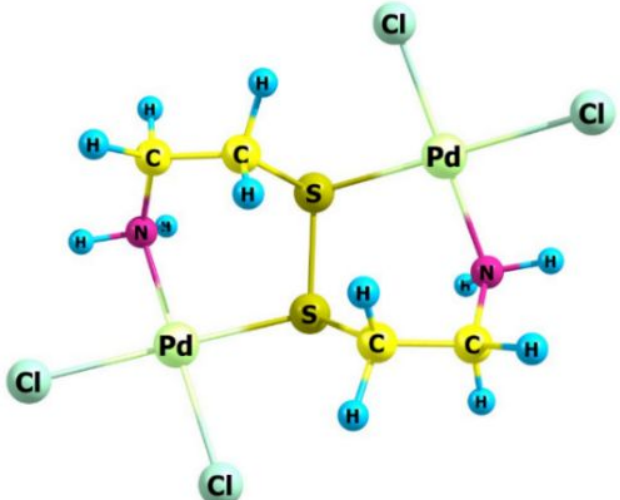

A

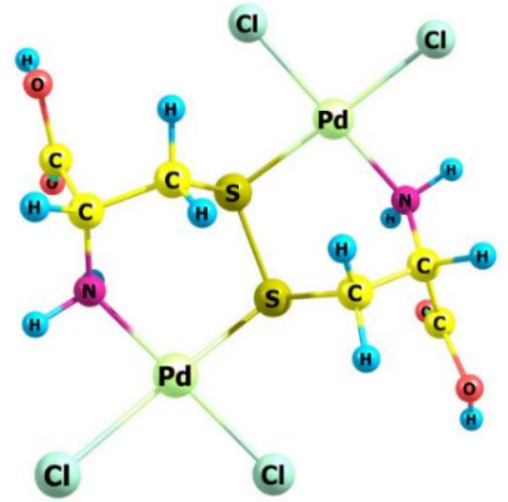

B

Figure S4. PBE0/Def2-SVP-D3 structures of chelate complexes of cystamine, $\left[\mathrm{Pd}_{2} \mathrm{Cl}_{4}(\mathrm{~S}, \mathrm{~N}-\mathrm{Cyst})\right]^{0}(\mathbf{A})$ and L-cystine, $\left[\mathrm{Pd}_{2} \mathrm{Cl}_{4}\left(\mathrm{~S}, \mathrm{~N}-\mathrm{H}_{2} \mathrm{CysS}\right)\right]^{0}(\mathbf{B})$ 

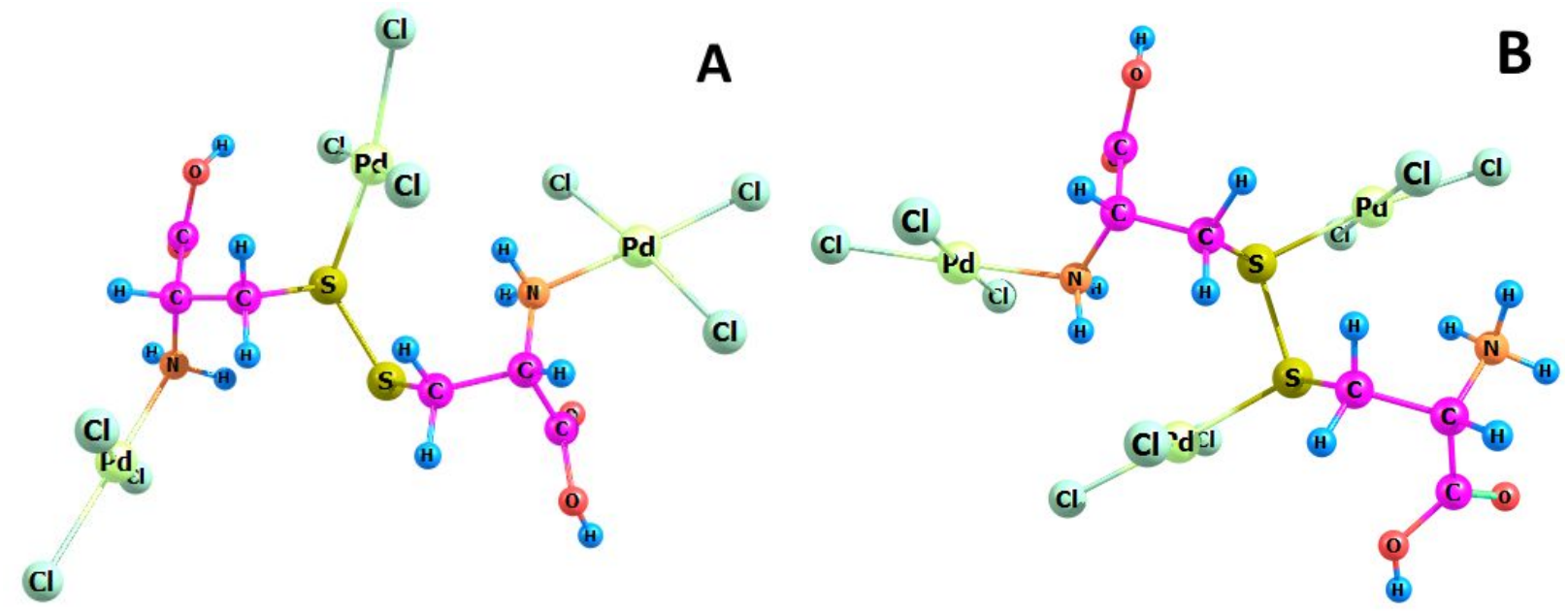

Cl

C Cl

D

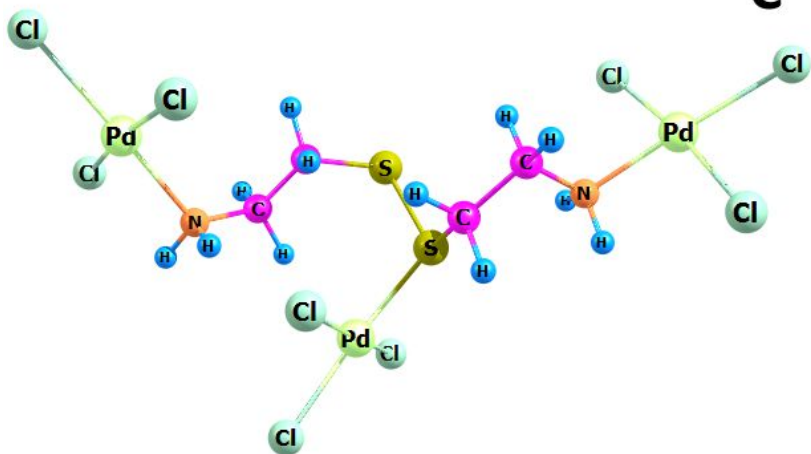

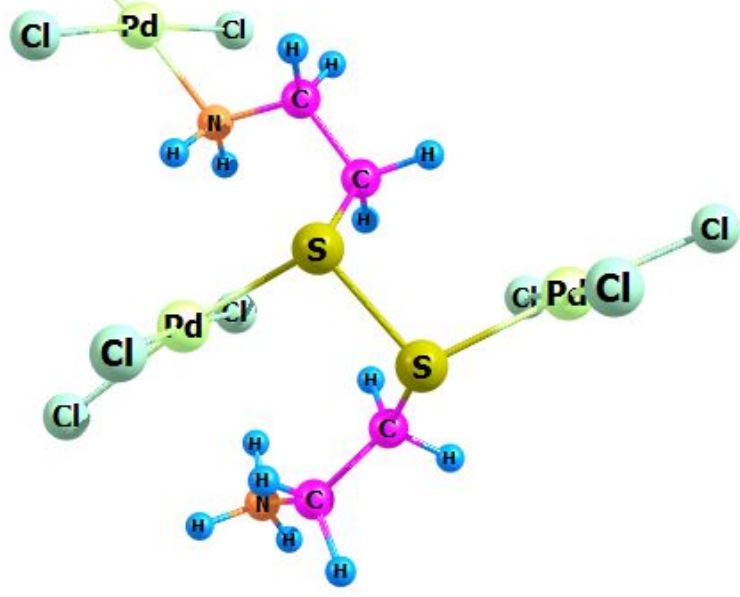

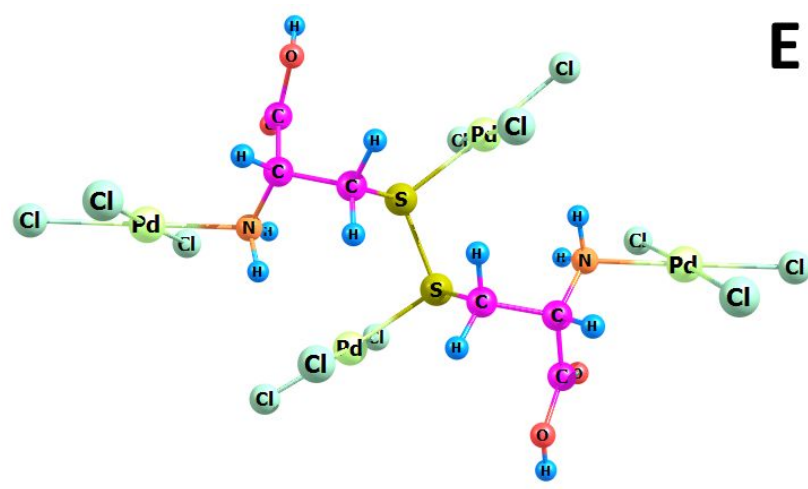

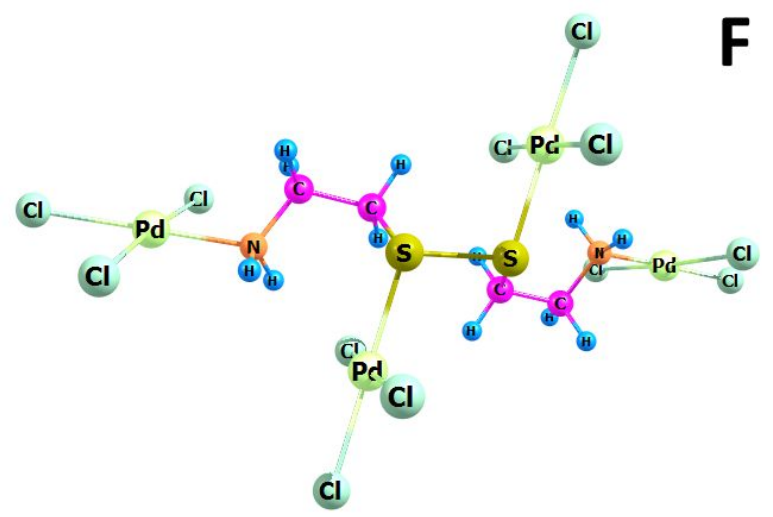

Figure S5. PBE0/Def2-SVP-D3 structures of the higher-order complexes of L-cystine (A, B, E) and cystamine $(\mathbf{C}, \mathbf{D}, \mathbf{F}):\left[\mathrm{Pd}_{3} \mathrm{Cl}_{9}\left(\mathrm{~S}, \mathrm{~N}, \mathrm{~N}^{\prime}-\mathrm{H}_{2} \mathrm{CysS}\right)\right]^{3-}(\mathbf{A}),\left[\mathrm{Pd}_{3} \mathrm{Cl}_{9}\left(\mathrm{~S}, \mathrm{~S}^{\prime}, \mathrm{N}^{-} \mathrm{H}_{3} \mathrm{CysS}\right)\right]^{2-}(\mathbf{B})$, $\left[\mathrm{Pd}_{3} \mathrm{Cl}_{9}\left(\mathrm{~S}, \mathrm{~N}, \mathrm{~N}^{\prime}-\mathrm{Cyst}\right)\right]^{3-}(\mathbf{C}),\left[\mathrm{Pd}_{3} \mathrm{Cl}_{9}\left(\mathrm{~S}, \mathrm{~S}^{\prime}, \mathrm{N}-\mathrm{HCyst}\right)\right]^{2-}(\mathbf{D}),\left[\mathrm{Pd}_{4} \mathrm{Cl}_{12}\left(\mathrm{~S}, \mathrm{~S}^{\prime}, \mathrm{N}^{\prime} \mathrm{N}^{\prime}-\mathrm{H}_{2} \mathrm{CysS}\right)\right]^{4-}(\mathbf{E})$, $\left[\mathrm{Pd}_{4} \mathrm{Cl}_{12}\left(\mathrm{~S}, \mathrm{~S}^{\prime}, \mathrm{N}, \mathrm{N}^{\prime}-\mathrm{HCyst}\right)\right]^{4-}(\mathbf{F})$ 

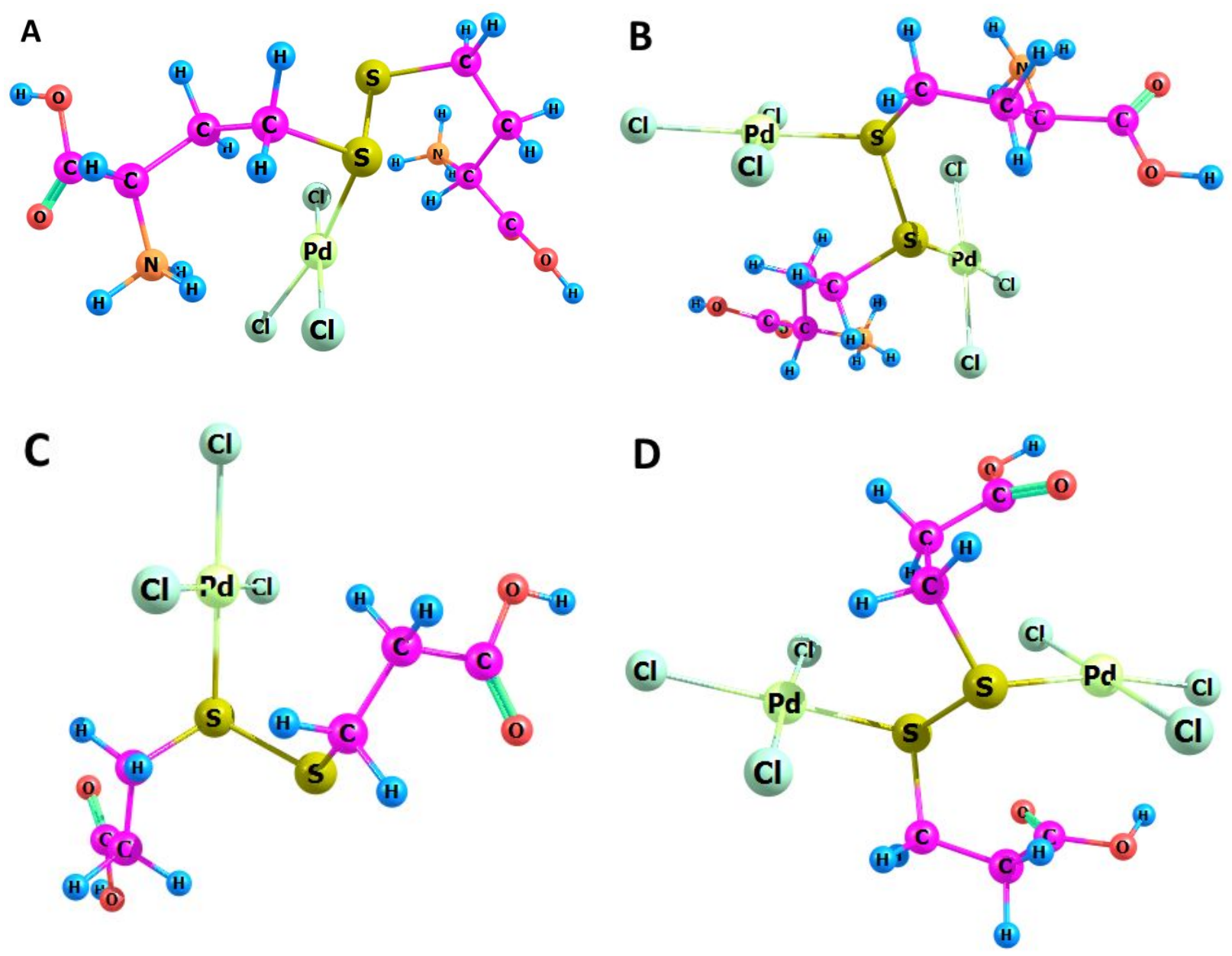

Figure S6. PBE0/Def2-SVP-D3 structures of mono- and binuclear complexes of DLhomocystine (A, B) and 3,3'-dithiodipropionic acid (C, D): $\left[\mathrm{PdCl}_{3}\left(\mathrm{~S}_{-} \mathrm{H}_{4} \mathrm{hCysS}\right)\right]^{+}(\mathbf{A})$, $\left[\mathrm{Pd}_{2} \mathrm{Cl}_{6}\left(\mathrm{~S}, \mathrm{~S}^{\prime}-\mathrm{H}_{4} \mathrm{hCysS}\right)\right]^{0}(\mathbf{B}),\left[\mathrm{PdCl}_{3}\left(\mathrm{~S}-\mathrm{H}_{2} \mathrm{DTPDA}\right)\right]^{-}(\mathbf{C}),\left[\mathrm{Pd}_{2} \mathrm{Cl}_{6}\left(\mathrm{~S}, \mathrm{~S}^{\prime}-\mathrm{H}_{2} \mathrm{DTPDA}\right)\right]^{2-}(\mathbf{D})$ 


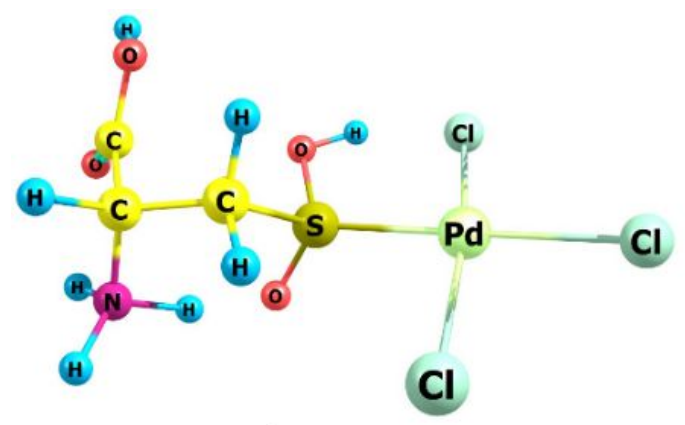

A

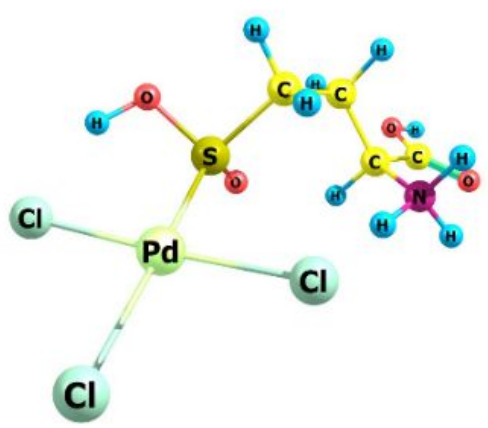

C

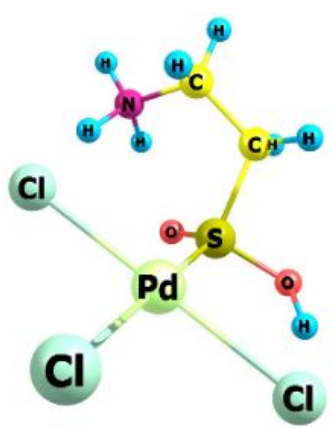

B

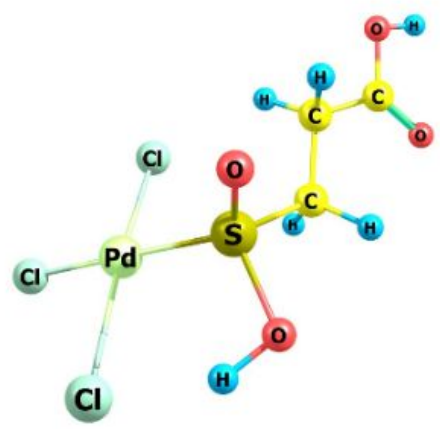

D

Figure S7. PBE0/Def2-SVP-D3 structures of sulfinic S-complexes: $\left[\mathrm{PdCl}_{3}\left(\mathrm{H}_{2} \mathrm{CysO}_{2} \mathrm{H}\right)\right](\mathbf{A})$, $\left[\mathrm{PdCl}_{3}\left(\mathrm{H}_{2} \mathrm{CysteaO}_{2} \mathrm{H}\right)\right](\mathbf{B}),\left[\mathrm{PdCl}_{3}\left(\mathrm{H}_{3} \mathrm{HCSA}\right)\right](\mathbf{C}),\left[\mathrm{PdCl}_{3}\left(\mathrm{H}_{2} \mathrm{SPA}\right)\right]^{-}(\mathbf{D})$

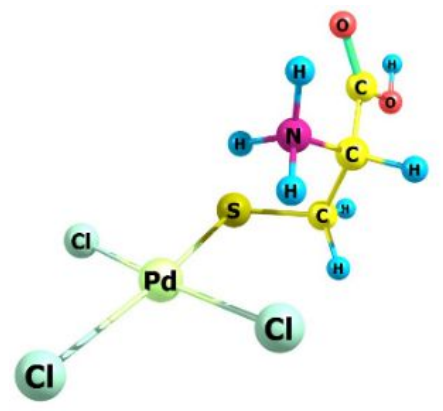

A

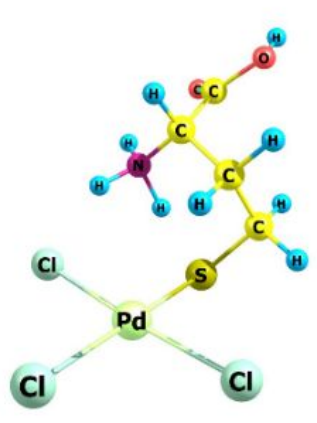

C

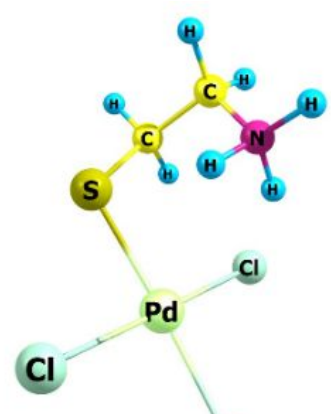

B

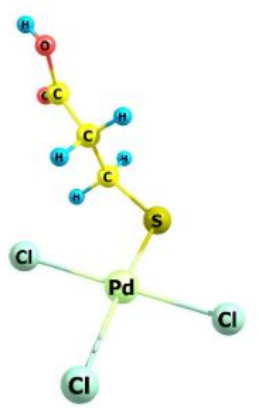

D

Figure S8. PBE0/Def2-SVP-D3 structures of thiolate S-complexes: $\left[\mathrm{PdCl}_{3}\left(\mathrm{H}_{2} \mathrm{Cys}\right)\right]^{-}(\mathbf{A})$, $\left[\mathrm{PdCl}_{3}(\mathrm{HCystea})\right]^{-}(\mathbf{B}),\left[\mathrm{PdCl}_{3}\left(\mathrm{H}_{2} \mathrm{HCys}\right)\right]^{-}(\mathbf{C}),\left[\mathrm{PdCl}_{3}(\mathrm{HMPA})\right]^{2-}(\mathbf{D})$ 
Optimized Geometries at the at the DEF2-SVP/PBE0-D3 Level of Theory

\begin{tabular}{|c|c|c|c|}
\hline \multicolumn{4}{|c|}{$\left[\mathrm{PdCl}_{3}\left(\mathrm{~S}-\mathrm{H}_{2} \mathrm{Cyst}\right)\right]^{+}$} \\
\hline $\mathrm{S}$ & 8.287687072 & -0.204443392 & 1.042803581 \\
\hline S & 6.466430673 & -0.886992063 & 1.885969728 \\
\hline $\mathrm{C}$ & 9.442347251 & -0.882795752 & 2.279770885 \\
\hline $\mathrm{C}$ & 9.518189513 & -0.331254628 & 3.685081710 \\
\hline $\mathrm{C}$ & 5.351775470 & -0.307010957 & 0.567252641 \\
\hline $\mathrm{C}$ & 4.832770107 & 1.113179002 & 0.794718108 \\
\hline $\mathrm{N}$ & 10.292146964 & 0.930191707 & 3.778463666 \\
\hline $\mathrm{N}$ & 4.747414250 & 1.899799518 & -0.469325032 \\
\hline $\mathrm{H}$ & 10.419878001 & -0.847815950 & 1.766748422 \\
\hline $\mathrm{H}$ & 9.169902185 & -1.948883891 & 2.327021380 \\
\hline $\mathrm{H}$ & 10.024796737 & -1.086138316 & 4.301556473 \\
\hline $\mathrm{H}$ & 8.525934139 & -0.146037698 & 4.118367686 \\
\hline $\mathrm{H}$ & 5.890702199 & -0.395132141 & -0.389804732 \\
\hline $\mathrm{H}$ & 4.546955316 & -1.056416682 & 0.533327084 \\
\hline $\mathrm{H}$ & 3.845434286 & 1.123189637 & 1.274251731 \\
\hline $\mathrm{H}$ & 5.520395225 & 1.666058517 & 1.448561191 \\
\hline $\mathrm{H}$ & 11.165261304 & 0.914245086 & 3.240708402 \\
\hline $\mathrm{H}$ & 10.539994494 & 1.131032588 & 4.753549499 \\
\hline $\mathrm{H}$ & 9.683928857 & 1.721696925 & 3.453739797 \\
\hline $\mathrm{H}$ & 3.881168075 & 1.755592281 & -1.001691191 \\
\hline $\mathrm{H}$ & 5.566606012 & 1.682101820 & -1.076906304 \\
\hline $\mathrm{H}$ & 4.879708767 & 2.900333515 & -0.262979139 \\
\hline $\mathrm{Pd}$ & 7.897546790 & 2.074160884 & 0.779488156 \\
\hline $\mathrm{Cl}$ & 6.986101668 & 4.155284987 & 0.276717306 \\
\hline $\mathrm{Cl}$ & 7.706570062 & 1.486621527 & -1.480403543 \\
\hline $\mathrm{Cl}$ & 7.907963346 & 2.622916115 & 3.043658347 \\
\hline \multicolumn{4}{|c|}{$\left[\mathrm{PdCl}_{3}\left(\mathrm{~S}-\mathrm{H}_{2} \mathrm{Cyst}\right)\right]^{+} \mathrm{SMD}$} \\
\hline $\mathrm{S}$ & 8.287687072 & -0.204443392 & 1.042803581 \\
\hline S & 6.466430673 & -0.886992063 & 1.885969728 \\
\hline $\mathrm{C}$ & 9.442347251 & -0.882795752 & 2.279770885 \\
\hline $\mathrm{C}$ & 9.518189513 & -0.331254628 & 3.685081710 \\
\hline $\mathrm{C}$ & 5.351775470 & -0.307010957 & 0.567252641 \\
\hline $\mathrm{C}$ & 4.832770107 & 1.113179002 & 0.794718108 \\
\hline $\mathrm{N}$ & 10.292146964 & 0.930191707 & 3.778463666 \\
\hline $\mathrm{N}$ & 4.747414250 & 1.899799518 & -0.469325032 \\
\hline $\mathrm{H}$ & 10.419878001 & -0.847815950 & 1.766748422 \\
\hline $\mathrm{H}$ & 9.169902185 & -1.948883891 & 2.327021380 \\
\hline $\mathrm{H}$ & 10.024796737 & -1.086138316 & 4.301556473 \\
\hline $\mathrm{H}$ & 8.525934139 & -0.146037698 & 4.118367686 \\
\hline $\mathrm{H}$ & 5.890702199 & -0.395132141 & -0.389804732 \\
\hline $\mathrm{H}$ & 4.546955316 & -1.056416682 & 0.533327084 \\
\hline $\mathrm{H}$ & 3.845434286 & 1.123189637 & 1.274251731 \\
\hline $\mathrm{H}$ & 5.520395225 & 1.666058517 & 1.448561191 \\
\hline $\mathrm{H}$ & 11.165261304 & 0.914245086 & 3.240708402 \\
\hline $\mathrm{H}$ & 10.539994494 & 1.131032588 & 4.753549499 \\
\hline $\mathrm{H}$ & 9.683928857 & 1.721696925 & 3.453739797 \\
\hline $\mathrm{H}$ & 3.881168075 & 1.755592281 & -1.001691191 \\
\hline $\mathrm{H}$ & 5.566606012 & 1.682101820 & -1.076906304 \\
\hline
\end{tabular}




$\begin{array}{lllr}\mathrm{H} & 4.879708767 & 2.900333515 & -0.262979139 \\ \mathrm{Pd} & 7.897546790 & 2.074160884 & 0.779488156 \\ \mathrm{Cl} & 6.986101668 & 4.155284987 & 0.276717306 \\ \mathrm{Cl} & 7.706570062 & 1.486621527 & -1.480403543 \\ \mathrm{Cl} & 7.907963346 & 2.622916115 & 3.043658347\end{array}$

\begin{tabular}{lrrr} 
& \multicolumn{3}{c}{$\left[\mathrm{PdCl}_{3}\left(\mathrm{~S}_{-}-\mathrm{H}_{4} \mathrm{CysS}\right)\right]^{+}$} \\
$\mathrm{S}$ & 0.665705319 & -0.674920685 & -0.408491330 \\
$\mathrm{~S}$ & -0.876549942 & -1.531233564 & -1.510853883 \\
$\mathrm{O}$ & 3.396750585 & 2.554914197 & 0.284715181 \\
$\mathrm{O}$ & -3.248031232 & -0.766890037 & 1.220437168 \\
$\mathrm{O}$ & 2.801983029 & 2.512934005 & -1.885536759 \\
$\mathrm{O}$ & -3.973616329 & -2.031209663 & -0.494419435 \\
$\mathrm{~N}$ & -2.946076056 & 1.397100886 & -0.267333200 \\
$\mathrm{~N}$ & 3.358426888 & -0.016399160 & 0.593565975 \\
$\mathrm{C}$ & 3.101392379 & 1.978154236 & -0.725337898 \\
$\mathrm{C}$ & -3.479128956 & -0.941572213 & 0.057088651 \\
$\mathrm{C}$ & 3.150674285 & 0.446306780 & -0.808332897 \\
$\mathrm{C}$ & -3.204412406 & 0.129768148 & -0.995942381 \\
$\mathrm{C}$ & 1.995384519 & -0.222747634 & -1.576082606 \\
$\mathrm{C}$ & -2.104896618 & -0.275923564 & -2.000667221 \\
$\mathrm{H}$ & -2.561613311 & 1.212968181 & 0.682491926 \\
$\mathrm{H}$ & 2.494312991 & 0.080740058 & 1.242924044 \\
$\mathrm{H}$ & -3.799549264 & 1.946425611 & -0.127506570 \\
$\mathrm{H}$ & -2.222137698 & 1.999271368 & -0.746431501 \\
$\mathrm{H}$ & 3.694540895 & -0.981715514 & 0.658351475 \\
$\mathrm{H}$ & 4.075379372 & 0.218206185 & -1.365672129 \\
$\mathrm{H}$ & -4.121453017 & 0.274291575 & -1.586976304 \\
$\mathrm{H}$ & 2.733654801 & 3.478688821 & -1.791873314 \\
$\mathrm{H}$ & -4.097712819 & -2.708555367 & 0.192472403 \\
$\mathrm{H}$ & 2.324923915 & -1.144054062 & -2.077198964 \\
$\mathrm{H}$ & -1.604342109 & 0.608173263 & -2.421482150 \\
$\mathrm{H}$ & 1.615270162 & 0.494519989 & -2.317566842 \\
$\mathrm{H}$ & -2.598776383 & -0.809675335 & -2.828493359 \\
$\mathrm{Pd}$ & 0.077189778 & 1.303538227 & 0.603721341 \\
$\mathrm{Cl}$ & -0.881228176 & 3.005834019 & 1.766270094 \\
$\mathrm{Cl}$ & 0.973201521 & 0.319962371 & 2.504992174 \\
$\mathrm{Cl}$ & -0.362114619 & 2.433736610 & -1.419289167 \\
$\mathrm{H}$ & 4.037390124 & 0.624140587 & 1.034271419
\end{tabular}

\begin{tabular}{lrrr}
\multicolumn{4}{c}{$\left[\mathrm{PdCl}_{3}\left(\mathrm{~S}-\mathrm{H}_{4} \mathrm{CysS}\right)\right]^{+} \mathrm{SMD}$} \\
$\mathrm{S}$ & 0.573938848 & -0.634782078 & -0.400257962 \\
$\mathrm{~S}$ & -0.897115633 & -1.442888905 & -1.638824993 \\
$\mathrm{O}$ & 3.207567717 & 2.570242860 & 0.309055245 \\
$\mathrm{O}$ & -2.925360771 & -0.843213823 & 1.154134128 \\
$\mathrm{O}$ & 2.863648271 & 2.462653728 & -1.911114360 \\
$\mathrm{O}$ & -3.949927639 & -2.047003743 & -0.450568060 \\
$\mathrm{~N}$ & -2.985995650 & 1.394495881 & -0.280333887 \\
$\mathrm{~N}$ & 3.363933785 & -0.047167696 & 0.620614106 \\
$\mathrm{C}$ & 3.036833511 & 1.951005039 & -0.712162907 \\
$\mathrm{C}$ & -3.366875374 & -0.974189557 & 0.039190226 \\
$\mathrm{C}$ & 3.115970385 & 0.428234866 & -0.768819106
\end{tabular}




$\begin{array}{lrrr}\mathrm{C} & -3.248186680 & 0.126250164 & -1.004888113 \\ \mathrm{C} & 1.965966656 & -0.252773124 & -1.522173237 \\ \mathrm{C} & -2.167628082 & -0.211460367 & -2.055039454 \\ \mathrm{H} & -2.529755447 & 1.187080264 & 0.625081291 \\ \mathrm{H} & 2.511766562 & -0.149566166 & 1.220922536 \\ \mathrm{H} & -3.852209434 & 1.911997358 & -0.065159047 \\ \mathrm{H} & -2.336252935 & 2.023460305 & -0.799599485 \\ \mathrm{H} & 3.926153799 & -0.904093137 & 0.666940212 \\ \mathrm{H} & 4.033462751 & 0.221994590 & -1.343903857 \\ \mathrm{H} & -4.195624541 & 0.230247948 & -1.551467907 \\ \mathrm{H} & 2.851062083 & 3.438290524 & -1.887096912 \\ \mathrm{H} & -3.959346354 & -2.776918746 & 0.199107519 \\ \mathrm{H} & 2.285253800 & -1.206840042 & -1.962460440 \\ \mathrm{H} & -1.710667293 & 0.696844796 & -2.470148759 \\ \mathrm{H} & 1.620909814 & 0.420937412 & -2.318044924 \\ \mathrm{H} & -2.657644616 & -0.735841598 & -2.892327944 \\ \mathrm{Pd} & 0.071554869 & 1.368933533 & 0.621919232 \\ \mathrm{Cl} & -0.584548726 & 3.228084339 & 1.796939358 \\ \mathrm{Cl} & 0.783559679 & 0.295923171 & 2.558763563 \\ \mathrm{Cl} & -0.338099961 & 2.497041317 & -1.393526275 \\ \mathrm{H} & 3.908198231 & 0.687799206 & 1.101064156\end{array}$

\begin{tabular}{lrrr} 
& \multicolumn{3}{c}{$\left[\mathrm{PdCl}_{3}\left(\mathrm{~N}-\mathrm{H}_{3} \mathrm{CysS}\right)\right]^{0}$} \\
$\mathrm{~S}$ & 0.223214263 & 1.114965713 & 0.247068889 \\
$\mathrm{~S}$ & -0.037511286 & -0.919922790 & 0.670765476 \\
$\mathrm{O}$ & 4.082769267 & 1.593948490 & 1.398711198 \\
$\mathrm{O}$ & -3.897766982 & -0.570631372 & 0.228502661 \\
$\mathrm{O}$ & 3.318343288 & 3.170097393 & 0.006447529 \\
$\mathrm{O}$ & -3.015279606 & -2.624930112 & 0.438543567 \\
$\mathrm{~N}$ & -2.193614234 & 0.236781904 & -1.548249212 \\
$\mathrm{~N}$ & 3.235588550 & -0.450525846 & -0.197397678 \\
$\mathrm{C}$ & 3.523397625 & 1.904031506 & 0.383501206 \\
$\mathrm{C}$ & -3.104730905 & -1.425200733 & -0.083676119 \\
$\mathrm{C}$ & 2.976431454 & 0.912459691 & -0.633186235 \\
$\mathrm{C}$ & -2.100348124 & -1.214727699 & -1.208379415 \\
$\mathrm{C}$ & 1.530061364 & 1.206958590 & -1.020867701 \\
$\mathrm{C}$ & -0.676768671 & -1.605850108 & -0.884478201 \\
$\mathrm{H}$ & -1.452890431 & 0.768187688 & -1.001898749 \\
$\mathrm{H}$ & 4.243525541 & -0.603430020 & -0.139088657 \\
$\mathrm{H}$ & -3.119277535 & 0.572680096 & -1.228337140 \\
$\mathrm{H}$ & -2.048495206 & 0.419565193 & -2.544404867 \\
$\mathrm{H}$ & 2.918969285 & -0.606976325 & 0.766310500 \\
$\mathrm{H}$ & 3.528467354 & 1.069274239 & -1.577139443 \\
$\mathrm{H}$ & -2.447868717 & -1.797516716 & -2.079688752 \\
$\mathrm{H}$ & 3.703248775 & 3.737907878 & 0.692495816 \\
$\mathrm{H}$ & -3.664050190 & -2.711029723 & 1.155880455 \\
$\mathrm{H}$ & 1.283868435 & 0.556392820 & -1.878683276 \\
$\mathrm{H}$ & 0.000877752 & -1.376763845 & -1.729705318 \\
$\mathrm{H}$ & 1.465935234 & 2.257406982 & -1.345739849 \\
$\mathrm{H}$ & -0.577757951 & -2.701461561 & -0.774053830 \\
$\mathrm{Pd}$ & 2.461541518 & -2.182776389 & -1.123497617 \\
$\mathrm{Cl}$ & 1.398249650 & -4.087884743 & -1.838746846
\end{tabular}




$\begin{array}{lllr}\mathrm{Cl} & 2.924345553 & -3.105229659 & 0.946033172 \\ \mathrm{Cl} & 1.974600070 & -1.121437251 & -3.184048815\end{array}$

\begin{tabular}{lrrr} 
& \multicolumn{4}{c}{$\left[\mathrm{PdCl}_{3}\left(\mathrm{~N}-\mathrm{H}_{3} \mathrm{CysS}\right)\right]^{0} \mathrm{SMD}$} \\
$\mathrm{S}$ & 0.261889965 & 1.058412949 & 0.172717680 \\
$\mathrm{~S}$ & 0.002875622 & -0.974907060 & 0.536332016 \\
$\mathrm{O}$ & 3.778154914 & 1.525729844 & 1.557243768 \\
$\mathrm{O}$ & -3.726580835 & -0.409552679 & 0.526324982 \\
$\mathrm{O}$ & 3.311738367 & 3.133549101 & 0.062010092 \\
$\mathrm{O}$ & -3.020577018 & -2.546902009 & 0.515535966 \\
$\mathrm{~N}$ & -2.308830939 & 0.281288074 & -1.593710677 \\
$\mathrm{~N}$ & 3.280104620 & -0.467781172 & -0.206669141 \\
$\mathrm{C}$ & 3.421116776 & 1.870512803 & 0.457065944 \\
$\mathrm{C}$ & -3.079021658 & -1.312400533 & 0.056490620 \\
$\mathrm{C}$ & 3.009681095 & 0.901550358 & -0.642374597 \\
$\mathrm{C}$ & -2.224391345 & -1.147263666 & -1.191086856 \\
$\mathrm{C}$ & 1.573873968 & 1.156110468 & -1.085419944 \\
$\mathrm{C}$ & -0.785416297 & -1.594847758 & -0.983929463 \\
$\mathrm{H}$ & -1.587377056 & 0.844165696 & -1.094905127 \\
$\mathrm{H}$ & 4.287862193 & -0.577729774 & -0.072905561 \\
$\mathrm{H}$ & -3.221245673 & 0.681842797 & -1.325949796 \\
$\mathrm{H}$ & -2.178375720 & 0.425699976 & -2.603692138 \\
$\mathrm{H}$ & 2.902967834 & -0.605324399 & 0.735922641 \\
$\mathrm{H}$ & 3.628535063 & 1.114752562 & -1.530345162 \\
$\mathrm{H}$ & -2.684096921 & -1.746530873 & -1.995691254 \\
$\mathrm{H}$ & 3.536503851 & 3.746953057 & 0.786461627 \\
$\mathrm{H}$ & -3.591188107 & -2.662928329 & 1.300078593 \\
$\mathrm{H}$ & 1.358413262 & 0.485334811 & -1.928524033 \\
$\mathrm{H}$ & -0.149959601 & -1.381050622 & -1.858203536 \\
$\mathrm{H}$ & 1.488907399 & 2.191623565 & -1.450480863 \\
$\mathrm{H}$ & -0.758767823 & -2.688772791 & -0.866008006 \\
$\mathrm{Pd}$ & 2.651327277 & -2.240144744 & -1.139555772 \\
$\mathrm{Cl}$ & 1.832389373 & -4.244458737 & -1.946709047 \\
$\mathrm{Cl}$ & 3.169483123 & -3.167700880 & 0.941296963 \\
$\mathrm{Cl}$ & 2.277079432 & -1.234866744 & -3.218327167 \\
& & & \\
& & &
\end{tabular}

$\left[\mathrm{PdCl}_{2}(\mathrm{~S}, \mathrm{~N}-\mathrm{HCyst})\right]^{+}$

$\begin{array}{lrrr}\mathrm{S} & 7.043491145 & 0.769223049 & 2.719547388 \\ \mathrm{~S} & 5.306575513 & -0.252281834 & 2.249261537 \\ \mathrm{C} & 8.271291920 & -0.608581138 & 2.779114258 \\ \mathrm{C} & 9.565741133 & -0.275540574 & 3.500327179 \\ \mathrm{C} & 5.354018296 & -0.485744218 & 0.445546876 \\ \mathrm{C} & 5.297142742 & 0.779397811 & -0.389534280 \\ \mathrm{~N} & 10.508906293 & 0.558991085 & 2.715150095 \\ \mathrm{~N} & 6.603416341 & 1.415524510 & -0.577942160 \\ \mathrm{H} & 8.450558843 & -0.992981250 & 1.762455590 \\ \mathrm{H} & 7.764620938 & -1.399911497 & 3.351409130 \\ \mathrm{H} & 10.071632011 & -1.219887937 & 3.751152160 \\ \mathrm{H} & 9.367367479 & 0.261885000 & 4.439984542 \\ \mathrm{H} & 6.192545034 & -1.139921318 & 0.154587639 \\ \mathrm{H} & 4.434808794 & -1.072582371 & 0.280806835 \\ \mathrm{H} & 4.885203773 & 0.519209909 & -1.380042051\end{array}$




$\begin{array}{lrrr}\mathrm{H} & 4.613037818 & 1.509142259 & 0.068182606 \\ \mathrm{H} & 10.693618371 & 0.185460198 & 1.777714818 \\ \mathrm{H} & 11.411583260 & 0.651509385 & 3.193478277 \\ \mathrm{H} & 10.102152490 & 1.566368648 & 2.608375793 \\ \mathrm{H} & 7.220243739 & 0.813841633 & -1.129411972 \\ \mathrm{H} & 6.520402163 & 2.270907736 & -1.139531648 \\ \mathrm{Pd} & 7.754221381 & 2.151986426 & 0.981342655 \\ \mathrm{Cl} & 8.631230200 & 3.483468909 & -0.640210050 \\ \mathrm{Cl} & 9.080608886 & 3.116356797 & 2.636598673\end{array}$

\begin{tabular}{lrrr} 
& \multicolumn{4}{c}{$\left[\mathrm{PdCl}_{2}(\mathrm{~S}, \mathrm{~N}-\mathrm{HCyst})\right]^{+} \mathrm{SMD}$} \\
$\mathrm{S}$ & 7.093998724 & 0.743906772 & 2.698201292 \\
$\mathrm{~S}$ & 5.281576203 & -0.151021073 & 2.280738654 \\
$\mathrm{C}$ & 8.306666838 & -0.640208963 & 2.695073453 \\
$\mathrm{C}$ & 9.563745049 & -0.282465112 & 3.465883114 \\
$\mathrm{C}$ & 5.261625374 & -0.445824818 & 0.488123586 \\
$\mathrm{C}$ & 5.354708387 & 0.781839837 & -0.391124432 \\
$\mathrm{~N}$ & 10.523990970 & 0.544920208 & 2.698015939 \\
$\mathrm{~N}$ & 6.721723828 & 1.297321257 & -0.537773985 \\
$\mathrm{H}$ & 8.514221921 & -0.975133387 & 1.667285654 \\
$\mathrm{H}$ & 7.794869419 & -1.454492977 & 3.228421145 \\
$\mathrm{H}$ & 10.073589710 & -1.211963039 & 3.753597363 \\
$\mathrm{H}$ & 9.328236510 & 0.258099534 & 4.394139453 \\
$\mathrm{H}$ & 6.003757831 & -1.213487602 & 0.214811749 \\
$\mathrm{H}$ & 4.270530275 & -0.911450614 & 0.351615820 \\
$\mathrm{H}$ & 4.981247306 & 0.501402593 & -1.390803736 \\
$\mathrm{H}$ & 4.705254413 & 1.586569657 & -0.015824382 \\
$\mathrm{H}$ & 10.778924781 & 0.128176566 & 1.791891499 \\
$\mathrm{H}$ & 11.405988864 & 0.667331191 & 3.214442397 \\
$\mathrm{H}$ & 10.145310537 & 1.509630979 & 2.539253121 \\
$\mathrm{H}$ & 7.330854755 & 0.570409360 & -0.934225002 \\
$\mathrm{H}$ & 6.728293502 & 2.052280428 & -1.231763644 \\
$\mathrm{Pd}$ & 7.702602211 & 2.219956376 & 1.023899283 \\
$\mathrm{Cl}$ & 8.305121145 & 3.796632249 & -0.552040950 \\
$\mathrm{Cl}$ & 8.967580014 & 3.233411791 & 2.706526500
\end{tabular}

\begin{tabular}{lrrr} 
& \multicolumn{3}{c}{$\left[\mathrm{PdCl}_{2}\left(\mathrm{~S}, \mathrm{~N}-\mathrm{H}_{3} \mathrm{CysS}\right)\right]^{+}$} \\
$\mathrm{S}$ & 0.510615050 & 1.258471436 & 0.048425075 \\
$\mathrm{~S}$ & -0.249312105 & -0.705396054 & 0.075813118 \\
$\mathrm{O}$ & 4.096748625 & 0.459469796 & 1.000471757 \\
$\mathrm{O}$ & -3.707039571 & -0.349020012 & 1.587073939 \\
$\mathrm{O}$ & 3.769177527 & 2.467549091 & 0.033780841 \\
$\mathrm{O}$ & -3.459477614 & -2.146228321 & 0.266658061 \\
$\mathrm{~N}$ & -2.824037152 & 1.369242639 & -0.258253660 \\
$\mathrm{~N}$ & 2.891324485 & -0.899885576 & -0.788535804 \\
$\mathrm{C}$ & 3.683986470 & 1.170201523 & 0.118966650 \\
$\mathrm{C}$ & -3.401666086 & -0.851265895 & 0.542188770 \\
$\mathrm{C}$ & 2.972252410 & 0.561762153 & -1.097962775 \\
$\mathrm{C}$ & -2.881795121 & -0.040723918 & -0.646458418 \\
$\mathrm{C}$ & 1.631064295 & 1.200937442 & -1.397233971 \\
$\mathrm{C}$ & -1.571563665 & -0.620484598 & -1.180274583 \\
$\mathrm{H}$ & -3.042723584 & 1.420596757 & 0.746682945
\end{tabular}




$\begin{array}{lrrr}\mathrm{H} & 1.907874421 & -1.189389179 & -0.595760813 \\ \mathrm{H} & -3.552310764 & 1.922285063 & -0.728845205 \\ \mathrm{H} & 3.275772035 & -1.500105793 & -1.524286367 \\ \mathrm{H} & 3.628817465 & 0.699304464 & -1.972919151 \\ \mathrm{H} & -3.615769988 & -0.165619799 & -1.461713979 \\ \mathrm{H} & 4.202077567 & 2.840621043 & 0.821847571 \\ \mathrm{H} & -3.813588843 & -2.613377245 & 1.042160667 \\ \mathrm{H} & 1.123501592 & 0.741916706 & -2.258218044 \\ \mathrm{H} & -1.223045919 & -0.056607003 & -2.059012791 \\ \mathrm{H} & 1.768795140 & 2.277076319 & -1.620084046 \\ \mathrm{H} & -1.734771893 & -1.667695372 & -1.479385930 \\ \mathrm{Pd} & -1.209398006 & 2.641177956 & -0.646805091 \\ \mathrm{Cl} & -2.816581441 & 4.052944101 & -1.414877814 \\ \mathrm{Cl} & 0.491407080 & 4.149730585 & -0.983830795 \\ \mathrm{H} & 3.450268898 & -1.012446194 & 0.092731781\end{array}$

$\left[\mathrm{PdCl}_{2}\left(\mathrm{~S}, \mathrm{~N}-\mathrm{H}_{3} \mathrm{CysS}\right)\right]^{+} \mathrm{SMD}$

$\begin{array}{lrrr}\mathrm{S} & 0.511876547 & 1.384604689 & 0.027607004 \\ \mathrm{~S} & -0.233418151 & -0.550254879 & 0.278524008 \\ \mathrm{O} & 4.091476407 & 0.386741137 & 1.047987071 \\ \mathrm{O} & -3.722303724 & -0.337337090 & 1.593834366 \\ \mathrm{O} & 3.681460315 & 2.429875534 & 0.186337067 \\ \mathrm{O} & -3.608902672 & -2.108868913 & 0.217288428 \\ \mathrm{~N} & -2.772611752 & 1.370323500 & -0.234016620 \\ \mathrm{~N} & 2.915532780 & -0.907160525 & -0.927100261 \\ \mathrm{C} & 3.651074765 & 1.115572123 & 0.194926788 \\ \mathrm{C} & -3.443625549 & -0.836320194 & 0.530757807 \\ \mathrm{C} & 2.990024541 & 0.573166020 & -1.068788244 \\ \mathrm{C} & -2.826906483 & -0.045672803 & -0.619333801 \\ \mathrm{C} & 1.655572168 & 1.224336686 & -1.393060501 \\ \mathrm{C} & -1.485011924 & -0.642105869 & -1.031763540 \\ \mathrm{H} & -2.899969640 & 1.414753458 & 0.788101941 \\ \mathrm{H} & 1.953409832 & -1.209659718 & -0.707040636 \\ \mathrm{H} & -3.587822122 & 1.861368715 & -0.615674633 \\ \mathrm{H} & 3.190579641 & -1.430975222 & -1.770930089 \\ \mathrm{H} & 3.673881323 & 0.795570944 & -1.906002144 \\ \mathrm{H} & -3.494823368 & -0.160724305 & -1.489559221 \\ \mathrm{H} & 4.127029049 & 2.792070158 & 0.977312200 \\ \mathrm{H} & -4.011845859 & -2.604314934 & 0.956076102 \\ \mathrm{H} & 1.153899182 & 0.738394617 & -2.242759771 \\ \mathrm{H} & -1.109129023 & -0.172285585 & -1.952386392 \\ \mathrm{H} & 1.823584351 & 2.276444675 & -1.673047242 \\ \mathrm{H} & -1.600927475 & -1.717669407 & -1.242438862 \\ \mathrm{Pd} & -1.245184834 & 2.692057829 & -0.747232167 \\ \mathrm{Cl} & -2.953996431 & 4.035447529 & -1.520344602 \\ \mathrm{Cl} & 0.369563801 & 4.258536882 & -1.261855821 \\ \mathrm{H} & 3.508115614 & -1.210872937 & -0.133076300\end{array}$

$\left[\mathrm{PdCl}_{2}\left(\mathrm{~N}, \mathrm{O}-\mathrm{H}_{3} \mathrm{CysS}\right)\right]^{+}$

$\begin{array}{lrrr}\mathrm{S} & -0.616964634 & 1.118117828 & 1.687375598 \\ \mathrm{~S} & 0.481433505 & -0.610703336 & 1.372811882 \\ \mathrm{O} & -4.616745910 & 1.320751098 & 0.428021341\end{array}$




$\begin{array}{lrrr}\mathrm{O} & 4.265555462 & -1.263739702 & 0.106321622 \\ \mathrm{O} & -3.549010806 & 2.606362217 & 1.899004752 \\ \mathrm{O} & 3.600889091 & -2.570335757 & 1.814208430 \\ \mathrm{~N} & -3.180705972 & -0.861110925 & 1.144022951 \\ \mathrm{~N} & 3.142889803 & 0.863188886 & 0.979949400 \\ \mathrm{C} & -3.866836025 & 1.442720040 & 1.371680713 \\ \mathrm{C} & 3.740893485 & -1.437351139 & 1.174482183 \\ \mathrm{C} & -3.247360940 & 0.244000005 & 2.088045405 \\ \mathrm{C} & 3.178696525 & -0.248858359 & 1.965772399 \\ \mathrm{C} & -1.949534439 & 0.571680232 & 2.818599758 \\ \mathrm{C} & 1.819853940 & -0.480678602 & 2.621676948 \\ \mathrm{H} & -2.367114914 & -0.798724577 & 0.529542983 \\ \mathrm{H} & 2.206835451 & 0.844913425 & 0.523941767 \\ \mathrm{H} & -3.192366764 & -1.788031573 & 1.575710951 \\ \mathrm{H} & 3.313683032 & 1.792588297 & 1.376935608 \\ \mathrm{H} & -3.999652753 & -0.024453721 & 2.853837789 \\ \mathrm{H} & 3.923561593 & -0.007334874 & 2.743200105 \\ \mathrm{H} & -4.037610253 & 3.306489119 & 1.430538771 \\ \mathrm{H} & 4.002509910 & -3.296381346 & 1.303660827 \\ \mathrm{H} & -1.591500726 & -0.296636495 & 3.389728194 \\ \mathrm{H} & 1.566001791 & 0.339494371 & 3.309164935 \\ \mathrm{H} & -2.103411993 & 1.404897585 & 3.520512934 \\ \mathrm{H} & 1.867661529 & -1.413933197 & 3.201942402 \\ \mathrm{Pd} & -4.896273249 & -0.814006384 & -0.090998349 \\ \mathrm{Cl} & -4.810793198 & -3.058942787 & -0.210156448 \\ \mathrm{Cl} & -6.714773852 & -0.614306549 & -1.385380589 \\ \mathrm{H} & 3.843006495 & 0.628526094 & 0.243724173\end{array}$

$\left[\mathrm{PdCl}_{2}\left(\mathrm{~N}, \mathrm{O}-\mathrm{H}_{3} \mathrm{CysS}\right)\right]^{+} \mathrm{SMD}$

$\begin{array}{lrrr}\mathrm{S} & -0.662304296 & 0.706144250 & 1.247357395 \\ \mathrm{~S} & 0.702173126 & -0.838371019 & 1.314203067 \\ \mathrm{O} & -4.759864941 & 1.322578737 & 0.571811257 \\ \mathrm{O} & 4.562637168 & -1.289896837 & 0.390902513 \\ \mathrm{O} & -3.317243385 & 2.471361631 & 1.822105503 \\ \mathrm{O} & 3.552798980 & -2.459048409 & 2.028518169 \\ \mathrm{~N} & -3.439530514 & -0.961730862 & 0.978846228 \\ \mathrm{~N} & 3.350835961 & 0.954674695 & 1.013735077 \\ \mathrm{C} & -3.844554162 & 1.363347655 & 1.391489987 \\ \mathrm{C} & 3.877489159 & -1.358985740 & 1.380469373 \\ \mathrm{C} & -3.274659009 & 0.092910448 & 1.992605749 \\ \mathrm{C} & 3.292397125 & -0.113013638 & 2.042305541 \\ \mathrm{C} & -1.867309174 & 0.237214786 & 2.549829675 \\ \mathrm{C} & 1.891738691 & -0.294616759 & 2.601598338 \\ \mathrm{H} & -2.706076856 & -0.891873184 & 0.262594444 \\ \mathrm{H} & 2.569219783 & 0.880604440 & 0.342244068 \\ \mathrm{H} & -3.373715495 & -1.895053306 & 1.394921189 \\ \mathrm{H} & 3.355589899 & 1.906935240 & 1.405934807 \\ \mathrm{H} & -3.950057094 & -0.146044371 & 2.837242935 \\ \mathrm{H} & 3.975871343 & 0.163422773 & 2.864719651 \\ \mathrm{H} & -3.723147496 & 3.269208315 & 1.424142101 \\ \mathrm{H} & 3.941152575 & -3.251873842 & 1.610029964 \\ \mathrm{H} & -1.531990020 & -0.711581412 & 2.992127378\end{array}$




$\begin{array}{lrrr}\mathrm{H} & 1.524248318 & 0.638479367 & 3.053106445 \\ \mathrm{H} & -1.847738153 & 1.000377257 & 3.341463314 \\ \mathrm{H} & 1.918821762 & -1.055019703 & 3.394484880 \\ \mathrm{Pd} & -5.299308684 & -0.672446152 & 0.102783809 \\ \mathrm{Cl} & -5.605242614 & -2.904041424 & -0.178229638 \\ \mathrm{Cl} & -7.311105913 & -0.121983088 & -0.837094562 \\ \mathrm{H} & 4.211689100 & 0.856520025 & 0.451630777\end{array}$

\begin{tabular}{lrrr} 
& \multicolumn{3}{c}{$\left[\mathrm{PdCl}_{2}\left(\mathrm{~S}, \mathrm{O}-\mathrm{H}_{4} \mathrm{CysS}\right)\right]^{2+}$} \\
$\mathrm{S}$ & 0.624607316 & -0.478891563 & 1.447150277 \\
$\mathrm{~S}$ & -1.328971364 & 0.179800348 & 1.087795446 \\
$\mathrm{O}$ & 4.371960123 & -2.648092852 & 0.470812412 \\
$\mathrm{O}$ & -4.039373606 & 3.546541131 & 0.477032987 \\
$\mathrm{O}$ & 3.412336447 & -0.692360488 & -0.030135012 \\
$\mathrm{O}$ & -3.799820192 & 1.324922032 & 0.167037964 \\
$\mathrm{~N}$ & 2.012785225 & -3.437498489 & 1.623146654 \\
$\mathrm{~N}$ & -1.395862981 & 3.401139559 & 1.359980699 \\
$\mathrm{C}$ & 3.349735390 & -1.863827913 & 0.250644532 \\
$\mathrm{C}$ & -3.364485010 & 2.438276386 & 0.244268835 \\
$\mathrm{C}$ & 2.031345659 & -2.642283520 & 0.346824882 \\
$\mathrm{C}$ & -1.866699555 & 2.731198033 & 0.115695258 \\
$\mathrm{C}$ & 0.793179641 & -1.784708139 & 0.157772295 \\
$\mathrm{C}$ & -1.088455996 & 1.465615632 & -0.200593130 \\
$\mathrm{H}$ & 1.843484419 & -2.837365363 & 2.446979571 \\
$\mathrm{H}$ & -0.334281635 & 3.427705211 & 1.403176637 \\
$\mathrm{H}$ & 5.222690068 & -2.171510543 & 0.386677766 \\
$\mathrm{H}$ & -4.992726052 & 3.360554510 & 0.560863573 \\
$\mathrm{H}$ & 1.272635346 & -4.154855963 & 1.614639237 \\
$\mathrm{H}$ & -1.786931159 & 4.348029927 & 1.451667916 \\
$\mathrm{H}$ & 2.067792453 & -3.384700892 & -0.470489762 \\
$\mathrm{H}$ & -1.714431421 & 3.449162399 & -0.707625301 \\
$\mathrm{H}$ & -0.122171028 & -2.396927357 & 0.178316369 \\
$\mathrm{H}$ & -0.011293147 & 1.684516236 & -0.281594257 \\
$\mathrm{H}$ & 0.863513784 & -1.313639230 & -0.834274638 \\
$\mathrm{H}$ & -1.449815824 & 1.071923285 & -1.161029447 \\
$\mathrm{Pd}$ & 2.325462184 & 1.049494075 & 0.764347073 \\
$\mathrm{Cl}$ & 1.484280115 & 2.775507009 & 1.979572326 \\
$\mathrm{Cl}$ & 3.957536445 & 2.383535947 & 0.037201633 \\
$\mathrm{H}$ & -1.663434808 & 2.866365619 & 2.198053546 \\
$\mathrm{H}$ & 2.914291286 & -3.923487817 & 1.763965439 \\
& & & \\
& & &
\end{tabular}

\begin{tabular}{lrrr} 
& \multicolumn{3}{c}{$\left[\mathrm{PdCl}_{2}\left(\mathrm{~S}, \mathrm{O}-\mathrm{H}_{4} \mathrm{CysS}\right)\right]^{2+} \mathrm{SMD}$} \\
$\mathrm{S}$ & 0.789515088 & -0.346704189 & 1.632023498 \\
$\mathrm{~S}$ & -1.147034159 & 0.360606436 & 1.383331627 \\
$\mathrm{O}$ & 4.315058066 & -2.751832590 & 0.362292555 \\
$\mathrm{O}$ & -4.148916030 & 3.371759104 & 0.278842083 \\
$\mathrm{O}$ & 3.517844765 & -0.679370591 & 0.160580497 \\
$\mathrm{O}$ & -3.740575316 & 1.157635206 & 0.203290751 \\
$\mathrm{~N}$ & 1.987206617 & -3.534312918 & 1.493500545 \\
$\mathrm{~N}$ & -1.522652254 & 3.496454932 & 1.294089346 \\
$\mathrm{C}$ & 3.353559277 & -1.880919332 & 0.282234716 \\
$\mathrm{C}$ & -3.376394428 & 2.305060086 & 0.196012544
\end{tabular}




$\begin{array}{lrrr}\mathrm{C} & 1.998203806 & -2.568353121 & 0.346097386 \\ \mathrm{C} & -1.901531295 & 2.690600376 & 0.099919723 \\ \mathrm{C} & 0.807055928 & -1.631613017 & 0.324867597 \\ \mathrm{C} & -1.013710796 & 1.472450290 & -0.064249329 \\ \mathrm{H} & 2.402646844 & -3.158720683 & 2.360554129 \\ \mathrm{H} & -0.488636874 & 3.608248691 & 1.328210839 \\ \mathrm{H} & 5.219726612 & -2.373479367 & 0.375511902 \\ \mathrm{H} & -5.092822529 & 3.134699800 & 0.363752682 \\ \mathrm{H} & 1.020599055 & -3.826267823 & 1.713863925 \\ \mathrm{H} & -1.957551995 & 4.431645864 & 1.305577646 \\ \mathrm{H} & 1.926112821 & -3.174977650 & -0.575857737 \\ \mathrm{H} & -1.755729255 & 3.343217723 & -0.776676281 \\ \mathrm{H} & -0.132305815 & -2.199669373 & 0.411288124 \\ \mathrm{H} & 0.035368509 & 1.787133583 & -0.161083051 \\ \mathrm{H} & 0.794037733 & -1.129555162 & -0.653443899 \\ \mathrm{H} & -1.312099803 & 0.941027170 & -0.977630205 \\ \mathrm{Pd} & 2.486304947 & 1.061306045 & 0.840459550 \\ \mathrm{Cl} & 1.589966530 & 2.937834846 & 1.785529676 \\ \mathrm{Cl} & 4.211240290 & 2.310870970 & 0.026131744 \\ \mathrm{H} & -1.769775717 & 3.035751418 & 2.182869395 \\ \mathrm{H} & 2.494171498 & -4.416389514 & 1.305989805\end{array}$

\begin{tabular}{lrrr} 
& \multicolumn{3}{c}{$\left[\mathrm{PdCl}\left(\mathrm{S}, \mathrm{N}, \mathrm{N}^{\prime}-\mathrm{H}_{2} \mathrm{CysS}\right)\right]^{+}$} \\
$\mathrm{S}$ & -1.017840793 & -0.875472353 & -0.753030661 \\
$\mathrm{~S}$ & 0.340504817 & -2.162021278 & -1.661099191 \\
$\mathrm{O}$ & -3.240813500 & 1.773138031 & 0.812225434 \\
$\mathrm{O}$ & -2.481243269 & -4.372450075 & -2.817227616 \\
$\mathrm{O}$ & -1.320669617 & 1.436116590 & 1.928448535 \\
$\mathrm{O}$ & -0.499100664 & -4.744952289 & -3.804942694 \\
$\mathrm{~N}$ & -2.553047634 & -1.802877930 & -3.453155826 \\
$\mathrm{~N}$ & -2.074855788 & 1.815669130 & -1.564380167 \\
$\mathrm{C}$ & -2.046956904 & 1.662608677 & 0.849766033 \\
$\mathrm{C}$ & -1.522547844 & -3.998094463 & -3.435885752 \\
$\mathrm{C}$ & -1.172622955 & 1.804156118 & -0.400145718 \\
$\mathrm{C}$ & -1.359858075 & -2.548286146 & -3.892649898 \\
$\mathrm{C}$ & -0.113445980 & 0.715951938 & -0.523357372 \\
$\mathrm{C}$ & -0.023583627 & -1.961690982 & -3.433188917 \\
$\mathrm{H}$ & -3.127382974 & -2.463399961 & -2.904876962 \\
$\mathrm{H}$ & -1.779313861 & 2.473953158 & -2.288273191 \\
$\mathrm{H}$ & -3.122592852 & -1.522468257 & -4.258399519 \\
$\mathrm{H}$ & -0.650879142 & 2.772880724 & -0.296849461 \\
$\mathrm{H}$ & -1.329595681 & -2.553492548 & -4.995653249 \\
$\mathrm{H}$ & -1.907427720 & 1.384839775 & 2.702476715 \\
$\mathrm{H}$ & -0.640961904 & -5.656090039 & -3.496488685 \\
$\mathrm{H}$ & 0.499699173 & 0.648395111 & 0.385915631 \\
$\mathrm{H}$ & 0.072973866 & -0.910037830 & -3.743280713 \\
$\mathrm{H}$ & 0.531368204 & 0.879671374 & -1.398566006 \\
$\mathrm{H}$ & 0.793440658 & -2.529198836 & -3.909210259 \\
$\mathrm{Pd}$ & -2.326146271 & -0.019527603 & -2.468306385 \\
$\mathrm{Cl}$ & -3.494346343 & 0.973431792 & -4.151291961 \\
$\mathrm{H}$ & -3.005600393 & 2.113731535 & -1.238236529
\end{tabular}




\begin{tabular}{lrrr}
\multicolumn{4}{c}{$\left[\mathrm{PdCl}\left(\mathrm{S}, \mathrm{N}, \mathrm{N}^{\prime}-\mathrm{H}_{2} \mathrm{CysS}\right)\right]^{+} \mathrm{SMD}$} \\
$\mathrm{S}$ & -1.029760504 & -0.821063536 & -0.816374839 \\
$\mathrm{~S}$ & 0.340133850 & -2.125448767 & -1.661540709 \\
$\mathrm{O}$ & -3.239444995 & 1.502278693 & 0.832933956 \\
$\mathrm{O}$ & -2.503883383 & -4.283960232 & -2.685870552 \\
$\mathrm{O}$ & -1.278187194 & 1.421987281 & 1.932608376 \\
$\mathrm{O}$ & -0.549318986 & -4.766697569 & -3.684949976 \\
$\mathrm{~N}$ & -2.540619218 & -1.756636590 & -3.538141537 \\
$\mathrm{~N}$ & -2.097437929 & 1.842719740 & -1.555780375 \\
$\mathrm{C}$ & -2.035024446 & 1.562876857 & 0.859209279 \\
$\mathrm{C}$ & -1.543359338 & -3.968263125 & -3.345521834 \\
$\mathrm{C}$ & -1.192467729 & 1.814953401 & -0.387905856 \\
$\mathrm{C}$ & -1.343322043 & -2.557691034 & -3.885064905 \\
$\mathrm{C}$ & -0.115827002 & 0.757723501 & -0.569573009 \\
$\mathrm{C}$ & -0.004200728 & -1.974783757 & -3.440825380 \\
$\mathrm{H}$ & -3.171857972 & -2.376250312 & -3.008099119 \\
$\mathrm{H}$ & -1.790166655 & 2.533887810 & -2.246917542 \\
$\mathrm{H}$ & -3.054354347 & -1.487249960 & -4.381494140 \\
$\mathrm{H}$ & -0.706179411 & 2.797326377 & -0.255064043 \\
$\mathrm{H}$ & -1.289036918 & -2.634902104 & -4.985555287 \\
$\mathrm{H}$ & -1.813562075 & 1.203104060 & 2.719879184 \\
$\mathrm{H}$ & -0.667924541 & -5.661468731 & -3.312463796 \\
$\mathrm{H}$ & 0.521738022 & 0.659454053 & 0.318923786 \\
$\mathrm{H}$ & 0.118003701 & -0.940586455 & -3.795425700 \\
$\mathrm{H}$ & 0.501323837 & 0.958764023 & -1.455787271 \\
$\mathrm{H}$ & 0.810493055 & -2.570004135 & -3.886338884 \\
$\mathrm{Pd}$ & -2.322845783 & 0.028926795 & -2.530374389 \\
$\mathrm{Cl}$ & -3.538545216 & 1.056877816 & -4.194184770 \\
$\mathrm{H}$ & -3.037213142 & 2.118609257 & -1.249965077
\end{tabular}

\begin{tabular}{lrrr} 
& \multicolumn{3}{c}{$\left[\mathrm{PdCl}\left(\mathrm{S}, \mathrm{N}, \mathrm{O}-\mathrm{H}_{3} \mathrm{CysS}\right)\right]^{2+}$} \\
$\mathrm{S}$ & -0.316416534 & 1.572089781 & 0.643833694 \\
$\mathrm{~S}$ & -1.557042646 & 0.025409794 & 0.048459976 \\
$\mathrm{O}$ & 3.114365148 & 4.332909235 & 0.557604589 \\
$\mathrm{O}$ & -4.556677415 & 1.953078030 & 1.152753824 \\
$\mathrm{O}$ & 1.050799860 & 4.147599669 & -0.260086440 \\
$\mathrm{O}$ & -4.960377554 & 0.154951005 & -0.137858480 \\
$\mathrm{~N}$ & -2.975159901 & 3.117032804 & -0.560807180 \\
$\mathrm{~N}$ & 2.887001634 & 1.769634988 & 1.508049842 \\
$\mathrm{C}$ & 2.086847130 & 3.657866865 & 0.124994798 \\
$\mathrm{C}$ & -4.409495975 & 1.311597204 & 0.148107012 \\
$\mathrm{C}$ & 2.379242612 & 2.149697333 & 0.143255220 \\
$\mathrm{C}$ & -3.496025674 & 1.801773022 & -0.983238934 \\
$\mathrm{C}$ & 1.229243886 & 1.274494056 & -0.325839996 \\
$\mathrm{C}$ & -2.451552879 & 0.755783911 & -1.361584364 \\
$\mathrm{H}$ & -3.392064897 & 3.291300154 & 0.374595657 \\
$\mathrm{H}$ & 2.135092485 & 1.766435480 & 2.216609807 \\
$\mathrm{H}$ & -3.320366349 & 3.864534654 & -1.176326213 \\
$\mathrm{H}$ & 3.306182333 & 0.827218157 & 1.507415545 \\
$\mathrm{H}$ & 3.230986800 & 2.004469704 & -0.545194281 \\
$\mathrm{H}$ & -4.124502406 & 1.949028872 & -1.878618041 \\
$\mathrm{H}$ & 2.964317088 & 5.300190422 & 0.529345747
\end{tabular}




$\begin{array}{lrrr}\mathrm{H} & -5.556219810 & -0.121089892 & 0.581765967 \\ \mathrm{H} & 1.480132010 & 0.205655637 & -0.234013929 \\ \mathrm{H} & -1.754206893 & 1.123917436 & -2.129294665 \\ \mathrm{H} & 1.049920131 & 1.483606243 & -1.391385418 \\ \mathrm{H} & -2.968791725 & -0.123406196 & -1.783877933 \\ \mathrm{Pd} & -1.009509826 & 3.551852870 & -0.447335895 \\ \mathrm{Cl} & -1.528557738 & 5.430930095 & -1.572080711 \\ \mathrm{H} & 3.617283450 & 2.428758633 & 1.827067768\end{array}$

\begin{tabular}{lrrr} 
& \multicolumn{3}{c}{$\left[\mathrm{PdCl}\left(\mathrm{S}, \mathrm{N}, \mathrm{O}-\mathrm{H}_{3} \mathrm{CysS}\right)\right]^{2+} \mathrm{SMD}$} \\
$\mathrm{S}$ & -0.376925650 & 1.618732671 & 0.719665557 \\
$\mathrm{~S}$ & -1.612618567 & 0.072470438 & 0.103890944 \\
$\mathrm{O}$ & 3.059971875 & 4.353630181 & 0.544196086 \\
$\mathrm{O}$ & -4.638104267 & 1.906536450 & 1.101262766 \\
$\mathrm{O}$ & 1.004877514 & 4.134895643 & -0.261163917 \\
$\mathrm{O}$ & -5.003626137 & 0.164456247 & -0.272140922 \\
$\mathrm{~N}$ & -2.959488091 & 3.117306292 & -0.516258925 \\
$\mathrm{~N}$ & 3.003036324 & 1.776193154 & 1.454369204 \\
$\mathrm{C}$ & 2.049032407 & 3.647416844 & 0.142962555 \\
$\mathrm{C}$ & -4.451625258 & 1.311219267 & 0.067462395 \\
$\mathrm{C}$ & 2.342703146 & 2.151845185 & 0.171324224 \\
$\mathrm{C}$ & -3.492786242 & 1.827953651 & -1.002271729 \\
$\mathrm{C}$ & 1.173535539 & 1.255737911 & -0.202466925 \\
$\mathrm{C}$ & -2.444587677 & 0.775778839 & -1.350439650 \\
$\mathrm{H}$ & -3.337442682 & 3.247098102 & 0.438049024 \\
$\mathrm{H}$ & 2.375794774 & 1.804418228 & 2.274406681 \\
$\mathrm{H}$ & -3.357952581 & 3.890375805 & -1.062789212 \\
$\mathrm{H}$ & 3.414458981 & 0.829295873 & 1.407032317 \\
$\mathrm{H}$ & 3.104640095 & 2.001977139 & -0.616660007 \\
$\mathrm{H}$ & -4.072587340 & 2.007863477 & -1.924646442 \\
$\mathrm{H}$ & 2.905399055 & 5.322373014 & 0.503847930 \\
$\mathrm{H}$ & -5.571271982 & -0.191047107 & 0.438997800 \\
$\mathrm{H}$ & 1.416254241 & 0.202608349 & -0.000980334 \\
$\mathrm{H}$ & -1.722791463 & 1.145948968 & -2.094129428 \\
$\mathrm{H}$ & 0.973924718 & 1.359368480 & -1.277507334 \\
$\mathrm{H}$ & -2.946224567 & -0.100591358 & -1.793063951 \\
$\mathrm{Pd}$ & -0.987596174 & 3.537804662 & -0.444741864 \\
$\mathrm{Cl}$ & -1.481226526 & 5.422778152 & -1.648628510 \\
$\mathrm{H}$ & 3.787672874 & 2.412875436 & 1.676738628 \\
& & & \\
& $\mathrm{C}$ & & \\
$\mathrm{S}$ & 6.767463333 & 1.124821169 & 2.914794150 \\
$\mathrm{C}$ & 5.649370801 & 0.688259212 & -0.517637552 \\
$\mathrm{H}$ & 9.641334604 & 1.481456875 & 2.061763394 \\
$\mathrm{H}$ & 6.168390097 & 1.998920650 & -0.065676796 \\
$\mathrm{C}$ & 8.280669194 & -0.779731552 & 2.884175196 \\
$\mathrm{C}$ & 7.975917239 & -0.199087616 & 4.551473437 \\
& 10.286983782 & 0.474678929 & 3.836317262
\end{tabular}




$\begin{array}{crrr}\mathrm{H} & 9.267070046 & 1.900861844 & 4.084093284 \\ \mathrm{H} & 6.947805852 & -0.766091261 & 0.477266932 \\ \mathrm{H} & 5.307653144 & -1.335180430 & 0.141154133 \\ \mathrm{H} & 6.161668385 & 0.433236753 & -1.457530003 \\ \mathrm{H} & 4.574182475 & 0.779088828 & -0.736099403 \\ \mathrm{H} & 10.135318909 & 0.776386598 & 1.506564054 \\ \mathrm{H} & 10.244276337 & 2.308575805 & 2.045199706 \\ \mathrm{H} & 6.414122173 & 2.578259232 & -0.874801831 \\ \mathrm{H} & 5.450159759 & 2.507731609 & 0.455467803 \\ \mathrm{Pd} & 7.896900985 & 1.867635434 & 1.032134656 \\ \mathrm{Cl} & 9.076539194 & 2.503941903 & -0.805176076\end{array}$

$\begin{array}{llll}\mathrm{S} & 5.511906898 & -0.532848728 & 2.150269823\end{array}$

$\begin{array}{llll}\text { C } & 8.281434584 & 0.042574912 & 3.480359683\end{array}$

$\begin{array}{llll}\text { C } & 9.377760057 & 1.080723110 & 3.565980080\end{array}$

$\begin{array}{llll}\text { C } & 5.799964456 & -0.515519300 & 0.353815139\end{array}$

$\begin{array}{llll}\text { C } & 5.362104093 & 0.764624022 & -0.335035393\end{array}$

$\begin{array}{llll}\mathrm{N} & 9.617405480 & 1.661390243 & 2.223244823\end{array}$

$\begin{array}{llll}\mathrm{N} & 6.378048065 & 1.843806950 & -0.324083464\end{array}$

$\begin{array}{llll}\mathrm{H} & 8.525192301 & -0.769713555 & 2.780362128\end{array}$

$\mathrm{H} \quad 8.035152578 \quad-0.380481592 \quad 4.465217526$

$\mathrm{H} \quad 10.298864353 \quad 0.622051050 \quad 3.958695749$

$\begin{array}{llll}\mathrm{H} & 9.098170430 & 1.893251798 & 4.253977580\end{array}$

$\begin{array}{llll}\mathrm{H} & 6.842180854 & -0.789788497 & 0.125815635\end{array}$

$\begin{array}{llll}\mathrm{H} & 5.162491186 & -1.350742537 & 0.015814601\end{array}$

$\begin{array}{llll}\mathrm{H} & 5.129696992 & 0.531797797 & -1.385659567\end{array}$

$\begin{array}{llll}\mathrm{H} & 4.438256065 & 1.137621615 & 0.127804934\end{array}$

$\begin{array}{llll}\mathrm{H} & 10.260292994 & 1.061485380 & 1.694903228\end{array}$

$\begin{array}{llll}\mathrm{H} & 10.092621263 & 2.564533269 & 2.293038373\end{array}$

$\begin{array}{llll}\mathrm{H} & 6.900544807 & 1.843979622 & -1.203956004\end{array}$

$\begin{array}{llll}\mathrm{H} & 5.924342431 & 2.762204157 & -0.285951357\end{array}$

$\begin{array}{lllr}\mathrm{Pd} & 7.945691518 & 1.799316480 & 1.016389504\end{array}$

$\begin{array}{llll}\mathrm{Cl} & 9.265778951 & 2.635325068 & -0.681568036\end{array}$

\begin{tabular}{lrrr} 
& \multicolumn{4}{c}{$\left[\mathrm{Pd}_{2} \mathrm{Cl}_{6}\left(\mathrm{~S}, \mathrm{~S}^{\prime}-\mathrm{H}_{4} \mathrm{CysS}\right)\right]^{0}$} \\
$\mathrm{~S}$ & 0.464536347 & -0.126165697 & 0.087814163 \\
$\mathrm{~S}$ & -1.066264001 & -1.397558050 & -0.635638156 \\
$\mathrm{O}$ & 4.383650832 & 1.923059630 & -0.093191939 \\
$\mathrm{O}$ & -4.739361732 & -1.213420176 & 0.290486749 \\
$\mathrm{O}$ & 2.630299047 & 2.651747854 & -1.306994776 \\
$\mathrm{O}$ & -4.551575946 & -1.779898337 & -1.879594437 \\
$\mathrm{~N}$ & -3.126221420 & 0.858337475 & 0.098584610 \\
$\mathrm{~N}$ & 3.404613245 & -0.550600074 & 0.022652212 \\
$\mathrm{C}$ & 3.404875455 & 1.736628437 & -0.759659854 \\
$\mathrm{C}$ & -4.300446202 & -1.049315704 & -0.817078234 \\
$\mathrm{C}$ & 2.958679016 & 0.306287627 & -1.096061413 \\
$\mathrm{C}$ & -3.350336557 & 0.095191273 & -1.147976880 \\
$\mathrm{C}$ & 1.486500749 & 0.069872203 & -1.426145320 \\
$\mathrm{C}$ & -2.041038902 & -0.357715675 & -1.778827216 \\
$\mathrm{H}$ & -2.622936393 & 0.269873114 & 0.777213073
\end{tabular}




$\begin{array}{lrrr}\mathrm{H} & 3.010849850 & -0.150375584 & 0.910040590 \\ \mathrm{H} & -4.010698087 & 1.121677315 & 0.544479924 \\ \mathrm{H} & -2.517245995 & 1.719023595 & -0.130253108 \\ \mathrm{H} & 3.018027355 & -1.542252904 & -0.097817569 \\ \mathrm{H} & 3.522596207 & 0.007665197 & -1.996419395 \\ \mathrm{H} & -3.848449405 & 0.767454971 & -1.866625113 \\ \mathrm{H} & 2.743605154 & 3.507647246 & -0.849006431 \\ \mathrm{H} & -5.084233984 & -2.553298305 & -1.632176331 \\ \mathrm{H} & 1.378157403 & -0.880720916 & -1.976066392 \\ \mathrm{H} & -1.444367986 & 0.509515533 & -2.093423962 \\ \mathrm{H} & 1.107179459 & 0.911851220 & -2.020561045 \\ \mathrm{H} & -2.244079182 & -1.023771099 & -2.640049932 \\ \mathrm{Pd} & 0.363436619 & 2.114342506 & 0.669274743 \\ \mathrm{Cl} & 0.996301442 & 4.270556707 & 1.060440908 \\ \mathrm{Cl} & 1.973717297 & 1.344796038 & 2.168753230 \\ \mathrm{Cl} & -1.204473199 & 2.861582987 & -0.919003608 \\ \mathrm{H} & 4.424406171 & -0.528447535 & 0.114317005 \\ \mathrm{Pd} & 0.062378703 & -3.105383078 & -1.825575259 \\ \mathrm{Cl} & 1.101207738 & -4.709040362 & -3.032468242 \\ \mathrm{Cl} & -1.744590848 & -3.184427347 & -3.257410479 \\ \mathrm{Cl} & 1.853128667 & -3.135094877 & -0.267173781\end{array}$

$\left[\mathrm{Pd}_{2} \mathrm{Cl}_{6}\left(\mathrm{~S}, \mathrm{~S}^{\prime}-\mathrm{H}_{4} \mathrm{CysS}\right)\right]^{0} \mathrm{SMD}$

$\begin{array}{lrrr}\mathrm{S} & 0.496871777 & -0.122461094 & -0.049576364 \\ \mathrm{~S} & -1.066358329 & -1.336897699 & -0.775590254 \\ \mathrm{O} & 4.325390110 & 1.950869226 & 0.102666465 \\ \mathrm{O} & -4.429660308 & -1.305303882 & 0.630599995 \\ \mathrm{O} & 2.842631672 & 2.575001193 & -1.474746353 \\ \mathrm{O} & -4.550476738 & -1.949855290 & -1.527462774 \\ \mathrm{~N} & -3.237807532 & 1.013256304 & 0.037868958 \\ \mathrm{~N} & 3.437344584 & -0.579329098 & 0.124444569 \\ \mathrm{C} & 3.473543064 & 1.704157795 & -0.710451636 \\ \mathrm{C} & -4.212154124 & -1.141619163 & -0.542734100 \\ \mathrm{C} & 3.059274250 & 0.271518051 & -1.025951362 \\ \mathrm{C} & -3.460292878 & 0.067426651 & -1.081443698 \\ \mathrm{C} & 1.621828606 & 0.025465946 & -1.477862750 \\ \mathrm{C} & -2.175731930 & -0.314588693 & -1.802147804 \\ \mathrm{H} & -2.837897204 & 0.540838912 & 0.861818784 \\ \mathrm{H} & 2.992516316 & -0.210658692 & 0.992626399 \\ \mathrm{H} & -4.113007705 & 1.453653379 & 0.357938774 \\ \mathrm{H} & -2.587872589 & 1.777162235 & -0.271214910 \\ \mathrm{H} & 3.085742664 & -1.553094747 & -0.022370205 \\ \mathrm{H} & 3.692182162 & -0.024060221 & -1.882899498 \\ \mathrm{H} & -4.107652239 & 0.569042837 & -1.819252570 \\ \mathrm{H} & 3.169256849 & 3.484483160 & -1.335887360 \\ \mathrm{H} & -5.038899849 & -2.731716405 & -1.205380024 \\ \mathrm{H} & 1.570989778 & -0.937574769 & -2.007839072 \\ \mathrm{H} & -1.640040967 & 0.568970690 & -2.175271300 \\ \mathrm{H} & 1.291263933 & 0.832871211 & -2.143577789 \\ \mathrm{H} & -2.401806757 & -0.977845883 & -2.654746526 \\ \mathrm{Pd} & 0.277388862 & 2.064465024 & 0.633877818 \\ \mathrm{Cl} & 0.374500009 & 4.203866584 & 1.446106120\end{array}$




$\begin{array}{lrrr}\mathrm{Cl} & 1.750817532 & 1.288902245 & 2.283636215 \\ \mathrm{Cl} & -0.976526025 & 2.790505840 & -1.191565495 \\ \mathrm{H} & 4.448986548 & -0.595058918 & 0.293579684 \\ \mathrm{Pd} & 0.057633805 & -2.986522811 & -2.065240357 \\ \mathrm{Cl} & 1.165025250 & -4.581840890 & -3.271934349 \\ \mathrm{Cl} & -1.563731549 & -2.873904664 & -3.704015505 \\ \mathrm{Cl} & 1.658555874 & -3.200499158 & -0.347143390\end{array}$

\begin{tabular}{lrrr} 
& \multicolumn{3}{c}{$\left[\mathrm{Pd}_{2} \mathrm{Cl}_{6}\left(\mathrm{~S}, \mathrm{~S}^{\prime}-\mathrm{H}_{2} \mathrm{Cyst}\right)\right]^{0}$} \\
$\mathrm{~S}$ & 8.120054991 & -0.072271931 & 1.019737463 \\
$\mathrm{~S}$ & 6.452724679 & -0.333021285 & 2.372196549 \\
$\mathrm{C}$ & 9.456491548 & -0.877914289 & 1.963522675 \\
$\mathrm{C}$ & 9.572147221 & -0.680747462 & 3.456633111 \\
$\mathrm{C}$ & 5.145903849 & -0.161808647 & 1.100384164 \\
$\mathrm{C}$ & 4.781968629 & 1.292385925 & 0.796735331 \\
$\mathrm{~N}$ & 9.738897178 & 0.730360082 & 3.878702272 \\
$\mathrm{~N}$ & 5.092402807 & 1.745119157 & -0.587427199 \\
$\mathrm{H}$ & 10.362541567 & -0.580052116 & 1.409206363 \\
$\mathrm{H}$ & 9.274561107 & -1.950279614 & 1.774549502 \\
$\mathrm{H}$ & 10.437874521 & -1.257208696 & 3.816805795 \\
$\mathrm{H}$ & 8.685784364 & -1.069822404 & 3.995580699 \\
$\mathrm{H}$ & 5.448158011 & -0.787502173 & 0.241208173 \\
$\mathrm{H}$ & 4.305157911 & -0.692245437 & 1.571292168 \\
$\mathrm{H}$ & 3.709074710 & 1.464587439 & 0.959251707 \\
$\mathrm{H}$ & 5.320711473 & 1.976583439 & 1.470315324 \\
$\mathrm{H}$ & 10.630217701 & 1.140744769 & 3.583239158 \\
$\mathrm{H}$ & 9.680833714 & 0.799724048 & 4.899931935 \\
$\mathrm{H}$ & 8.978279860 & 1.400909620 & 3.498697511 \\
$\mathrm{H}$ & 4.328889018 & 1.586814853 & -1.248795743 \\
$\mathrm{H}$ & 5.974007970 & 1.329634057 & -0.993970365 \\
$\mathrm{H}$ & 5.363548584 & 2.763793697 & -0.564275921 \\
$\mathrm{Pd}$ & 7.967007948 & 2.203097715 & 0.638586126 \\
$\mathrm{Cl}$ & 6.902474824 & 4.179637543 & 0.019467654 \\
$\mathrm{Cl}$ & 7.959469893 & 1.515031754 & -1.589186635 \\
$\mathrm{Cl}$ & 7.949301780 & 2.874562270 & 2.868049360 \\
$\mathrm{Pd}$ & 6.589422060 & -2.520967587 & 2.966982817 \\
$\mathrm{Cl}$ & 6.767825556 & -4.671796794 & 3.667839629 \\
$\mathrm{Cl}$ & 6.620929229 & -3.114635788 & 0.721539127 \\
$\mathrm{Cl}$ & 6.777354129 & -1.669814478 & 5.148138168
\end{tabular}

\begin{tabular}{lccc} 
& \multicolumn{4}{c}{$\left[\mathrm{Pd}_{2} \mathrm{Cl}_{6}\left(\mathrm{~S}_{2} \mathrm{~S}^{\prime}-\mathrm{H}_{2} \mathrm{Cyst}\right)\right]^{0} \mathrm{SMD}$} \\
$\mathrm{S}$ & 8.145832987 & -0.216945743 & 1.100844380 \\
$\mathrm{~S}$ & 6.415371212 & -0.530279492 & 2.327697143 \\
$\mathrm{C}$ & 9.473473275 & -0.824829191 & 2.195270613 \\
$\mathrm{C}$ & 9.584756178 & -0.407035534 & 3.641550641 \\
$\mathrm{C}$ & 5.133876220 & -0.201904806 & 1.056475171 \\
$\mathrm{C}$ & 4.802852815 & 1.283770993 & 0.903730111 \\
$\mathrm{~N}$ & 9.944422294 & 1.013032262 & 3.843067294 \\
$\mathrm{~N}$ & 5.009567918 & 1.807680566 & -0.475147373 \\
$\mathrm{H}$ & 10.377537784 & -0.593239359 & 1.605710916 \\
$\mathrm{H}$ & 9.350585464 & -1.919424265 & 2.167720132 \\
$\mathrm{H}$ & 10.373850699 & -1.022339978 & 4.098550595
\end{tabular}




$\begin{array}{lrrr}\mathrm{H} & 8.659087382 & -0.618115954 & 4.199128867 \\ \mathrm{H} & 5.433684723 & -0.723837097 & 0.133764505 \\ \mathrm{H} & 4.274776872 & -0.766729165 & 1.448878336 \\ \mathrm{H} & 3.759909188 & 1.486902386 & 1.178965639 \\ \mathrm{H} & 5.434973854 & 1.884995081 & 1.571343699 \\ \mathrm{H} & 10.816959752 & 1.288093164 & 3.372528638 \\ \mathrm{H} & 10.072676127 & 1.220174840 & 4.844130208 \\ \mathrm{H} & 9.186194181 & 1.658336298 & 3.496929142 \\ \mathrm{H} & 4.211514706 & 1.629044581 & -1.101607787 \\ \mathrm{H} & 5.861295313 & 1.419057046 & -0.935586384 \\ \mathrm{H} & 5.192923643 & 2.824472087 & -0.446879826 \\ \mathrm{Pd} & 7.992665518 & 2.027925656 & 0.557113212 \\ \mathrm{Cl} & 7.367190309 & 4.111595980 & -0.238918624 \\ \mathrm{Cl} & 7.947003249 & 1.193325380 & -1.621116715 \\ \mathrm{Cl} & 7.757252141 & 2.821781605 & 2.728351798 \\ \mathrm{Pd} & 6.447690023 & -2.797065950 & 2.687138483 \\ \mathrm{Cl} & 6.322015559 & -5.050771355 & 3.179971409 \\ \mathrm{Cl} & 6.273171743 & -3.203615138 & 0.405380231 \\ \mathrm{Cl} & 6.770905700 & -2.231157230 & 4.929952461\end{array}$

\begin{tabular}{lccc} 
& \multicolumn{3}{c}{$\left[\mathrm{Pd}_{2} \mathrm{Cl}_{6}\left(\mathrm{~N}, \mathrm{~N}^{\prime}-\mathrm{Cyst}\right)\right]^{2-}$} \\
$\mathrm{S}$ & 8.217984213 & 1.440791150 & 1.594085149 \\
$\mathrm{~S}$ & 6.397413928 & 0.925776287 & 2.427606288 \\
$\mathrm{C}$ & 9.244951153 & -0.038295159 & 1.886553744 \\
$\mathrm{C}$ & 9.390782567 & -0.440004404 & 3.337888895 \\
$\mathrm{C}$ & 5.569326106 & -0.018610836 & 1.108501781 \\
$\mathrm{C}$ & 4.968985855 & 0.829086564 & -0.003862699 \\
$\mathrm{~N}$ & 10.028034273 & 0.613019990 & 4.123362463 \\
$\mathrm{~N}$ & 4.244692595 & 0.008675323 & -0.972153639 \\
$\mathrm{H}$ & 10.243982120 & 0.220315797 & 1.490824030 \\
$\mathrm{H}$ & 8.854287487 & -0.877877459 & 1.291743652 \\
$\mathrm{H}$ & 10.032528583 & -1.333832509 & 3.365017710 \\
$\mathrm{H}$ & 8.395211556 & -0.686749974 & 3.750845920 \\
$\mathrm{H}$ & 6.271818434 & -0.771165549 & 0.713077246 \\
$\mathrm{H}$ & 4.771397007 & -0.566586604 & 1.641439955 \\
$\mathrm{H}$ & 4.257924949 & 1.561782509 & 0.406316828 \\
$\mathrm{H}$ & 5.757558153 & 1.379187192 & -0.537909504 \\
$\mathrm{H}$ & 9.865076477 & 0.493325473 & 5.123596851 \\
$\mathrm{H}$ & 9.606687045 & 1.520731135 & 3.902538772 \\
$\mathrm{H}$ & 3.543646635 & -0.562446336 & -0.498941548 \\
$\mathrm{H}$ & 4.867028481 & -0.584570005 & -1.529892905 \\
$\mathrm{Pd}$ & 12.096108055 & 0.968836650 & 4.118958069 \\
$\mathrm{Pd}$ & 3.152518735 & 0.986557274 & -2.434114807 \\
$\mathrm{Cl}$ & 14.335128097 & 1.462354282 & 4.256842256 \\
$\mathrm{Cl}$ & 12.469967601 & -0.649086378 & 2.464701673 \\
$\mathrm{Cl}$ & 11.489495719 & 2.470365928 & 5.816631048 \\
$\mathrm{Cl}$ & 1.920915860 & 2.036427519 & -4.068278194 \\
$\mathrm{Cl}$ & 1.710439610 & 1.583788870 & -0.680268026 \\
$\mathrm{Cl}$ & 4.760355299 & 0.119122598 & -3.904378961
\end{tabular}

$\left[\mathrm{Pd}_{2} \mathrm{Cl}_{6}\left(\mathrm{~N}, \mathrm{~N}^{\prime}-\mathrm{Cyst}\right)\right]^{2-} \mathrm{SMD}$ 


$\begin{array}{ccrr}\mathrm{S} & 6.442661510 & 0.931983475 & 2.600208513 \\ \mathrm{C} & 9.214461439 & -0.075819408 & 1.753898205 \\ \mathrm{C} & 9.343414351 & -0.636758082 & 3.148600072 \\ \mathrm{C} & 5.508589262 & -0.037102286 & 1.377172084 \\ \mathrm{C} & 5.079573583 & 0.778070375 & 0.170824639 \\ \mathrm{~N} & 9.891333428 & 0.332630079 & 4.100117448 \\ \mathrm{~N} & 4.306981606 & -0.015496123 & -0.791573198 \\ \mathrm{H} & 10.202856602 & 0.239084272 & 1.378006447 \\ \mathrm{H} & 8.828846309 & -0.850377469 & 1.074006662 \\ \mathrm{H} & 10.023271383 & -1.498912531 & 3.107694038 \\ \mathrm{H} & 8.358969675 & -0.987639526 & 3.502921069 \\ \mathrm{H} & 6.089931336 & -0.929090526 & 1.090766203 \\ \mathrm{H} & 4.630759163 & -0.392198822 & 1.945818571 \\ \mathrm{H} & 4.453450934 & 1.625148693 & 0.484835478 \\ \mathrm{H} & 5.960678330 & 1.181906934 & -0.350075939 \\ \mathrm{H} & 9.913357885 & -0.076616488 & 5.036369119 \\ \mathrm{H} & 9.285149392 & 1.156260277 & 4.169963552 \\ \mathrm{H} & 3.521270619 & -0.472482935 & -0.321364577 \\ \mathrm{H} & 4.886061584 & -0.750184554 & -1.207966316 \\ \mathrm{Pd} & 11.772447916 & 1.177407473 & 3.833887001 \\ \mathrm{Pd} & 3.518553392 & 1.087849861 & -2.340396920 \\ \mathrm{Cl} & 13.874309434 & 2.155803036 & 3.722354219 \\ \mathrm{Cl} & 12.538810772 & -0.736190182 & 2.736313152 \\ \mathrm{Cl} & 10.831682584 & 3.046276885 & 4.872629695 \\ \mathrm{Cl} & 2.611646406 & 2.337268530 & -4.067997561 \\ \mathrm{Cl} & 1.985233809 & 1.885149556 & -0.765645890 \\ \mathrm{Cl} & 5.202782803 & 0.185799943 & -3.684674366\end{array}$

\begin{tabular}{lrrr} 
& \multicolumn{4}{c}{$\left[\mathrm{Pd}_{2} \mathrm{Cl}_{6}\left(\mathrm{~N}^{\prime} \mathrm{N}^{\prime}-\mathrm{H}_{2} \mathrm{CysS}\right)\right]^{2-}$} \\
$\mathrm{S}$ & 0.555039401 & -0.861446898 & -0.273766571 \\
$\mathrm{~S}$ & -0.555039401 & 0.861446898 & -0.273766571 \\
$\mathrm{O}$ & 3.366556801 & -3.639685478 & 0.007198221 \\
$\mathrm{O}$ & -3.366556801 & 3.639685478 & 0.007198221 \\
$\mathrm{O}$ & 1.942699139 & -3.315557841 & 1.696512947 \\
$\mathrm{O}$ & -1.942699139 & 3.315557841 & 1.696512947 \\
$\mathrm{~N}$ & 3.787252748 & -0.926313013 & -0.316910913 \\
$\mathrm{~N}$ & -3.787252748 & 0.926313013 & -0.316910913 \\
$\mathrm{C}$ & 2.802439488 & -2.889647805 & 0.764261090 \\
$\mathrm{C}$ & -2.802439488 & 2.889647805 & 0.764261090 \\
$\mathrm{C}$ & 3.029674953 & -1.397668968 & 0.830607353 \\
$\mathrm{C}$ & -3.029674953 & 1.397668968 & 0.830607353 \\
$\mathrm{C}$ & 1.775290463 & -0.570677840 & 1.063392293 \\
$\mathrm{C}$ & -1.775290463 & 0.570677840 & 1.063392293 \\
$\mathrm{H}$ & 3.182402318 & -0.752115517 & -1.126138901 \\
$\mathrm{H}$ & -3.182402318 & 0.752115517 & -1.126138901 \\
$\mathrm{H}$ & 4.437833028 & -1.652184187 & -0.623667670 \\
$\mathrm{H}$ & -4.437833028 & 1.652184187 & -0.623667670 \\
$\mathrm{H}$ & 3.660629255 & -1.207554933 & 1.727543372 \\
$\mathrm{H}$ & -3.660629255 & 1.207554933 & 1.727543372 \\
$\mathrm{H}$ & 1.860622790 & -4.273681137 & 1.580110694 \\
$\mathrm{H}$ & -1.860622790 & 4.273681137 & 1.580110694 \\
$\mathrm{H}$ & 2.071126365 & 0.489201521 & 1.080294306
\end{tabular}




$\begin{array}{lrrr}\mathrm{H} & -2.071126365 & -0.489201521 & 1.080294306 \\ \mathrm{H} & 1.327011752 & -0.838738434 & 2.029351816 \\ \mathrm{H} & -1.327011752 & 0.838738434 & 2.029351816 \\ \mathrm{Pd} & 4.925858354 & 0.837178115 & -0.179012870 \\ \mathrm{Pd} & -4.925858354 & -0.837178115 & -0.179012870 \\ \mathrm{Cl} & 6.198805051 & 2.747003676 & -0.155392214 \\ \mathrm{Cl} & -6.198805051 & -2.747003676 & -0.155392214 \\ \mathrm{Cl} & 4.908428278 & 0.684104176 & -2.507136664 \\ \mathrm{Cl} & -4.908428278 & -0.684104176 & -2.507136664 \\ \mathrm{Cl} & 4.803794441 & 0.780223380 & 2.169344549 \\ \mathrm{Cl} & -4.803794441 & -0.780223380 & 2.169344549\end{array}$

\begin{tabular}{lrrr} 
& \multicolumn{3}{c}{$\left[\mathrm{Pd}_{2} \mathrm{Cl}_{6}\left(\mathrm{~N}, \mathrm{~N}^{\prime}-\mathrm{H}_{2} \mathrm{CysS}\right)\right]^{2-} \mathrm{SMD}$} \\
$\mathrm{S}$ & -0.272007932 & 1.666622986 & 0.277907417 \\
$\mathrm{~S}$ & 0.461498853 & -0.174135628 & -0.272776288 \\
$\mathrm{O}$ & -3.981340032 & 3.461037090 & -0.148359590 \\
$\mathrm{O}$ & 2.387169775 & -3.719855284 & -0.320618562 \\
$\mathrm{O}$ & -2.624955778 & 3.831482065 & 1.598671257 \\
$\mathrm{O}$ & 1.111460440 & -3.207712881 & 1.452735526 \\
$\mathrm{~N}$ & -3.314424209 & 0.816772567 & -0.240467917 \\
$\mathrm{~N}$ & 3.493587397 & -1.223782260 & -0.421732602 \\
$\mathrm{C}$ & -3.247994858 & 3.053184978 & 0.721283254 \\
$\mathrm{C}$ & 2.034303983 & -2.941596692 & 0.533409850 \\
$\mathrm{C}$ & -2.984595649 & 1.576644140 & 0.959418800 \\
$\mathrm{C}$ & 2.649254238 & -1.568840625 & 0.718759039 \\
$\mathrm{C}$ & -1.593637857 & 1.260285214 & 1.479077695 \\
$\mathrm{C}$ & 1.667565807 & -0.466185549 & 1.072270983 \\
$\mathrm{H}$ & -2.583415168 & 0.952529934 & -0.947775480 \\
$\mathrm{H}$ & 2.928943390 & -1.024962817 & -1.253148418 \\
$\mathrm{H}$ & -4.165167495 & 1.202969389 & -0.660688228 \\
$\mathrm{H}$ & 4.075273004 & -2.031921916 & -0.660611241 \\
$\mathrm{H}$ & -3.687829748 & 1.264698780 & 1.755186542 \\
$\mathrm{H}$ & 3.318308389 & -1.649568521 & 1.595807747 \\
$\mathrm{H}$ & -2.853662495 & 4.769138091 & 1.454612215 \\
$\mathrm{H}$ & 0.782714812 & -4.119966447 & 1.350199539 \\
$\mathrm{H}$ & -1.535206016 & 0.189964138 & 1.725159527 \\
$\mathrm{H}$ & 2.239174061 & 0.456679550 & 1.246945818 \\
$\mathrm{H}$ & -1.397842476 & 1.834814837 & 2.394002357 \\
$\mathrm{H}$ & 1.133569268 & -0.736341290 & 1.993093583 \\
$\mathrm{Pd}$ & -3.471706915 & -1.257350429 & -0.174907095 \\
$\mathrm{Pd}$ & 4.686111958 & 0.480781583 & -0.295845409 \\
$\mathrm{Cl}$ & -3.572686658 & -3.566699728 & -0.209249010 \\
$\mathrm{Cl}$ & 6.014030433 & 2.373367188 & -0.260539890 \\
$\mathrm{Cl}$ & -2.911782127 & -1.116758338 & -2.439828256 \\
$\mathrm{Cl}$ & 4.190063173 & 0.744408922 & -2.561026991 \\
$\mathrm{Cl}$ & -4.039173787 & -1.210404469 & 2.093103279 \\
$\mathrm{Cl}$ & 5.064400217 & 0.080701423 & 1.979112221 \\
& &
\end{tabular}

$\left[\mathrm{Pd}_{2} \mathrm{Cl}_{6}(\mathrm{~S}, \mathrm{~N}-\mathrm{HCyst})\right]$

$\begin{array}{llll}\mathrm{S} & 7.797401826 & 1.689976816 & 2.068067158 \\ \mathrm{~S} & 5.828405184 & 1.243416549 & 2.661107760 \\ \mathrm{C} & 8.664806120 & 0.110969145 & 1.788570555\end{array}$




$\begin{array}{lrrr}\mathrm{C} & 8.798967189 & -0.838835190 & 2.965196901 \\ \mathrm{C} & 5.138038946 & 0.389355931 & 1.220530737 \\ \mathrm{C} & 5.207244585 & 1.254273802 & -0.025385449 \\ \mathrm{~N} & 9.205462864 & -0.204323979 & 4.241372516 \\ \mathrm{~N} & 4.694681248 & 0.516933366 & -1.173396968 \\ \mathrm{H} & 9.646179340 & 0.464331524 & 1.425821733 \\ \mathrm{H} & 8.199163910 & -0.443586497 & 0.958716392 \\ \mathrm{H} & 9.519053138 & -1.635737390 & 2.726037516 \\ \mathrm{H} & 7.827565694 & -1.323228442 & 3.159027973 \\ \mathrm{H} & 5.598295343 & -0.604773318 & 1.120805201 \\ \mathrm{H} & 4.076712238 & 0.233251274 & 1.490848883 \\ \mathrm{H} & 4.554788445 & 2.127063900 & 0.125817396 \\ \mathrm{H} & 6.241255001 & 1.599660294 & -0.206333757 \\ \mathrm{H} & 10.209633524 & -0.042415663 & 4.330519410 \\ \mathrm{H} & 8.829743878 & -0.794639144 & 5.043580665 \\ \mathrm{H} & 8.686478601 & 0.701299880 & 4.431571079 \\ \mathrm{H} & 5.289455637 & -0.269213058 & -1.464492124 \\ \mathrm{H} & 4.597439156 & 1.135655348 & -1.978078539 \\ \mathrm{Pd} & 6.176472881 & -0.147385631 & 4.510090387 \\ \mathrm{Pd} & 2.880591817 & -0.503848308 & -1.031185875 \\ \mathrm{Cl} & 7.204824382 & -1.494644897 & 6.141532172 \\ \mathrm{Cl} & 5.365497366 & -1.999333054 & 3.424095889 \\ \mathrm{Cl} & 7.183466262 & 1.754847035 & 5.479924876 \\ \mathrm{Cl} & 0.921090654 & -1.677738315 & -0.960454997 \\ \mathrm{Cl} & 2.003256907 & 1.320216838 & 0.145803454 \\ \mathrm{Cl} & 4.102920380 & -2.190930619 & -2.119590782\end{array}$

$\left[\mathrm{Pd}_{2} \mathrm{Cl}_{6}(\mathrm{~S}, \mathrm{~N}-\mathrm{HCyst})\right]^{-} \mathrm{SMD}$

$\begin{array}{lrrr}\mathrm{S} & 7.779485972 & 1.699768010 & 2.073653005 \\ \mathrm{~S} & 5.837126482 & 1.179502454 & 2.637640223 \\ \mathrm{C} & 8.743892044 & 0.170033946 & 1.824877718 \\ \mathrm{C} & 8.894726384 & -0.794183337 & 2.982327777 \\ \mathrm{C} & 5.165153561 & 0.321229558 & 1.189976149 \\ \mathrm{C} & 5.210447471 & 1.197122862 & -0.044714903 \\ \mathrm{~N} & 9.322221635 & -0.166034963 & 4.256695534 \\ \mathrm{~N} & 4.642904977 & 0.491136524 & -1.197939813 \\ \mathrm{H} & 9.714699141 & 0.594144089 & 1.512762210 \\ \mathrm{H} & 8.361944780 & -0.390737965 & 0.959714281 \\ \mathrm{H} & 9.629767114 & -1.566818657 & 2.714030133 \\ \mathrm{H} & 7.939426388 & -1.302966468 & 3.181432909 \\ \mathrm{H} & 5.669369357 & -0.648336571 & 1.071548121 \\ \mathrm{H} & 4.120522155 & 0.120319823 & 1.478292532 \\ \mathrm{H} & 4.614917275 & 2.104553356 & 0.126015651 \\ \mathrm{H} & 6.247366736 & 1.498146277 & -0.266244660 \\ \mathrm{H} & 10.295897326 & 0.153226814 & 4.254263193 \\ \mathrm{H} & 9.196287179 & -0.822824255 & 5.040505488 \\ \mathrm{H} & 8.703120936 & 0.635966184 & 4.514214633 \\ \mathrm{H} & 5.231516101 & -0.303207897 & -1.465928447 \\ \mathrm{H} & 4.624747316 & 1.117015075 & -2.009592099 \\ \mathrm{Pd} & 6.103459087 & -0.220040088 & 4.481618055 \\ \mathrm{Pd} & 2.796418278 & -0.438880222 & -1.043080098 \\ \mathrm{Cl} & 6.804293806 & -1.516817237 & 6.270170757\end{array}$




$\begin{array}{rrrr}\mathrm{Cl} & 5.194179204 & -2.051767629 & 3.388098604 \\ \mathrm{Cl} & 7.035951476 & 1.662247943 & 5.517650313 \\ \mathrm{Cl} & 0.755787501 & -1.527222035 & -0.962756603 \\ \mathrm{Cl} & 1.966369104 & 1.421287346 & 0.106473910 \\ \mathrm{Cl} & 3.846893732 & -2.245244737 & -2.091584408\end{array}$

\begin{tabular}{lrrr} 
& \multicolumn{3}{c}{$\left[\mathrm{Pd}_{2} \mathrm{Cl}_{6}\left(\mathrm{~S}, \mathrm{~N}_{-}-\mathrm{H}_{3} \mathrm{CysS}\right)\right]^{-}$} \\
$\mathrm{S}$ & 0.968683483 & 1.628969200 & 0.301300167 \\
$\mathrm{~S}$ & -0.058344214 & -0.182353089 & 0.578239269 \\
$\mathrm{O}$ & 4.433584424 & 0.855391708 & 1.728227313 \\
$\mathrm{O}$ & -3.922353620 & -1.493010190 & 0.916405330 \\
$\mathrm{O}$ & 4.230375345 & 2.582017067 & 0.323042083 \\
$\mathrm{O}$ & -2.271041427 & -2.653809139 & -0.057890382 \\
$\mathrm{~N}$ & -3.087482058 & 0.822058859 & -0.074469051 \\
$\mathrm{~N}$ & 3.172484921 & -0.875637130 & 0.008023351 \\
$\mathrm{C}$ & 4.074240609 & 1.299990185 & 0.672356603 \\
$\mathrm{C}$ & -2.985805811 & -1.567686866 & 0.161244451 \\
$\mathrm{C}$ & 3.411800029 & 0.488676919 & -0.434520838 \\
$\mathrm{C}$ & -2.527079416 & -0.393922378 & -0.694757405 \\
$\mathrm{C}$ & 2.178368535 & 1.188492077 & -0.990727920 \\
$\mathrm{C}$ & -1.030703939 & -0.342804868 & -0.955848679 \\
$\mathrm{H}$ & -2.494584933 & 1.203883223 & 0.715418433 \\
$\mathrm{H}$ & 3.958767698 & -1.203382515 & 0.587515286 \\
$\mathrm{H}$ & -4.007629350 & 0.604263388 & 0.325822578 \\
$\mathrm{H}$ & -3.154225455 & 1.661699284 & -0.718405788 \\
$\mathrm{H}$ & 2.337403472 & -0.923653934 & 0.594031630 \\
$\mathrm{H}$ & 4.135424339 & 0.457470205 & -1.269825175 \\
$\mathrm{H}$ & -3.018465765 & -0.526605051 & -1.675850444 \\
$\mathrm{H}$ & 4.652447871 & 3.027046371 & 1.073645930 \\
$\mathrm{H}$ & -2.614358349 & -3.372436615 & 0.496055152 \\
$\mathrm{H}$ & 1.704325044 & 0.558227743 & -1.765168300 \\
$\mathrm{H}$ & -0.788288786 & 0.501303397 & -1.621964495 \\
$\mathrm{H}$ & 2.433065798 & 2.156970301 & -1.442277332 \\
$\mathrm{H}$ & -0.673422686 & -1.249785907 & -1.472148001 \\
$\mathrm{Pd}$ & -0.760730500 & 2.941757413 & -0.552530487 \\
$\mathrm{Pd}$ & 3.108946815 & -2.524888591 & -1.305416064 \\
$\mathrm{Cl}$ & -2.849214528 & 3.620178713 & -1.361805907 \\
$\mathrm{Cl}$ & 3.122039287 & -4.418866101 & -2.583960734 \\
$\mathrm{Cl}$ & 0.096148761 & 2.937170341 & -2.677981464 \\
$\mathrm{Cl}$ & 4.779647997 & -3.316483832 & 0.118221617 \\
$\mathrm{Cl}$ & -1.600181327 & 2.847591775 & 1.652004188 \\
$\mathrm{Cl}$ & 1.431330397 & -1.587501127 & -2.664008465 \\
& & &
\end{tabular}

\section{$\left[\mathrm{Pd}_{2} \mathrm{Cl}_{6}\left(\mathrm{~S}, \mathrm{~N}-\mathrm{H}_{3} \mathrm{CysS}\right)\right]^{-\mathrm{SMD}}$}

$\begin{array}{lrrr}\mathrm{S} & 0.955497110 & 1.674106932 & 0.282323178 \\ \mathrm{~S} & -0.049807024 & -0.147408122 & 0.564339332 \\ \mathrm{O} & 4.507944734 & 0.681495965 & 1.681197299 \\ \mathrm{O} & -3.854199220 & -1.525897371 & 0.996658419 \\ \mathrm{O} & 4.187466232 & 2.542569007 & 0.468235186 \\ \mathrm{O} & -2.115746723 & -2.638331899 & 0.099362161 \\ \mathrm{~N} & -3.130433147 & 0.777227232 & -0.112600933 \\ \mathrm{~N} & 3.132100299 & -0.871492499 & -0.054360387\end{array}$




$\begin{array}{lrrr}\mathrm{C} & 4.089053215 & 1.239671335 & 0.696521635 \\ \mathrm{C} & -2.908327116 & -1.595623473 & 0.252054113 \\ \mathrm{C} & 3.404291685 & 0.508731483 & -0.454294508 \\ \mathrm{C} & -2.525035375 & -0.448089554 & -0.681049318 \\ \mathrm{C} & 2.192946620 & 1.261086257 & -0.988476844 \\ \mathrm{C} & -1.036245987 & -0.341439776 & -0.964414889 \\ \mathrm{H} & -2.557117843 & 1.191509255 & 0.658487555 \\ \mathrm{H} & 3.862478077 & -1.163587802 & 0.604866773 \\ \mathrm{H} & -4.038084528 & 0.528715159 & 0.309243321 \\ \mathrm{H} & -3.257267619 & 1.548789664 & -0.793269359 \\ \mathrm{H} & 2.253234351 & -0.910590122 & 0.469385382 \\ \mathrm{H} & 4.133925982 & 0.510056674 & -1.284635547 \\ \mathrm{H} & -3.035304601 & -0.658527309 & -1.638325241 \\ \mathrm{H} & 4.654810354 & 2.991101194 & 1.199040507 \\ \mathrm{H} & -2.402779029 & -3.395498616 & 0.646017938 \\ \mathrm{H} & 1.723234000 & 0.696947102 & -1.807752789 \\ \mathrm{H} & -0.820159028 & 0.503256494 & -1.637570965 \\ \mathrm{H} & 2.495810245 & 2.241550458 & -1.379962451 \\ \mathrm{H} & -0.677927795 & -1.245286341 & -1.475734113 \\ \mathrm{Pd} & -0.763230378 & 2.971987745 & -0.613456334 \\ \mathrm{Pd} & 3.082548034 & -2.506148164 & -1.336977760 \\ \mathrm{Cl} & -2.774796639 & 3.829098051 & -1.385550139 \\ \mathrm{Cl} & 3.006913013 & -4.422237025 & -2.624702954 \\ \mathrm{Cl} & 0.084298006 & 2.902374394 & -2.756959569 \\ \mathrm{Cl} & 4.517594636 & -3.472028816 & 0.233907497 \\ \mathrm{Cl} & -1.575548055 & 2.947823598 & 1.586577299 \\ \mathrm{Cl} & 1.623036178 & -1.455580274 & -2.826127045\end{array}$

$\left[\mathrm{Pd}_{3} \mathrm{Cl}_{9}(\mathrm{~S}, \mathrm{~S} \text { ',N-HCyst })\right]^{2-}$

$\begin{array}{crrr}\mathrm{S} & 7.879871981 & 1.242331512 & 2.606165363 \\ \mathrm{~S} & 5.730338590 & 1.126775931 & 2.740396387 \\ \mathrm{C} & 8.431048556 & -0.497400770 & 2.762728601 \\ \mathrm{C} & 9.136649277 & -0.744002299 & 4.083060020 \\ \mathrm{C} & 5.245648241 & 0.479123125 & 1.100837467 \\ \mathrm{C} & 5.138617816 & 1.577564834 & 0.050350889 \\ \mathrm{~N} & 10.364103066 & 0.028989437 & 4.264611396 \\ \mathrm{~N} & 5.711759810 & 1.149293580 & -1.255105544 \\ \mathrm{H} & 9.091052069 & -0.678225593 & 1.901383581 \\ \mathrm{H} & 7.549793351 & -1.155387307 & 2.652171149 \\ \mathrm{H} & 9.416386404 & -1.810247951 & 4.090055462 \\ \mathrm{H} & 8.472702134 & -0.564463986 & 4.942287740 \\ \mathrm{H} & 5.956331619 & -0.316731214 & 0.829668857 \\ \mathrm{H} & 4.296801024 & -0.037022275 & 1.302843214 \\ \mathrm{H} & 4.098196671 & 1.894570815 & -0.109797063 \\ \mathrm{H} & 5.713525236 & 2.468225747 & 0.352416025 \\ \mathrm{H} & 10.905254576 & 0.061026632 & 3.399655933 \\ \mathrm{H} & 10.195885925 & 0.977112104 & 4.614178744 \\ \mathrm{H} & 5.037710373 & 0.726048274 & -1.892601707 \\ \mathrm{H} & 6.519563663 & 0.477023951 & -1.087683545 \\ \mathrm{Pd} & 8.188343647 & 2.076077327 & 0.453415094 \\ \mathrm{Pd} & 5.059709590 & -0.502536005 & 4.232056776 \\ \mathrm{Cl} & 8.016710356 & 3.028141449 & -1.711373744\end{array}$




$\begin{array}{rrrr}\mathrm{Cl} & 8.362082632 & -0.074730450 & -0.526265406 \\ \mathrm{Cl} & 7.801102200 & 4.127994252 & 1.404930406 \\ \mathrm{Cl} & 4.110963345 & -1.963529608 & 5.712615673 \\ \mathrm{Cl} & 4.787720162 & 1.271313082 & 5.690797649 \\ \mathrm{Cl} & 5.365504710 & -2.235131447 & 2.668756533 \\ \mathrm{Pd} & 11.711111698 & -0.782504872 & 5.628575358 \\ \mathrm{Cl} & 13.225222302 & -1.672526656 & 7.117162771 \\ \mathrm{Cl} & 11.977361760 & -2.501509589 & 4.046186528 \\ \mathrm{Cl} & 11.280963844 & 1.124413064 & 6.908558053 \\ \mathrm{H} & 6.246653426 & 1.948090633 & -1.699347560\end{array}$

\begin{tabular}{cccc} 
& \multicolumn{3}{c}{$\left[\mathrm{Pd}_{3} \mathrm{Cl}_{9}\left(\mathrm{~S}_{,} \mathrm{S}^{\prime}, \mathrm{N}-\mathrm{HCyst}\right)\right]^{2-} \mathrm{SMD}$} \\
$\mathrm{S}$ & 7.261511762 & 1.025817199 & 3.514568958 \\
$\mathrm{~S}$ & 5.277926357 & 0.962967132 & 2.716166564 \\
$\mathrm{C}$ & 7.674522672 & -0.703770214 & 3.922817173 \\
$\mathrm{C}$ & 9.056385175 & -0.762012892 & 4.561982585 \\
$\mathrm{C}$ & 5.477908485 & 0.324883724 & 1.021849573 \\
$\mathrm{C}$ & 5.803933337 & 1.463494180 & 0.062686762 \\
$\mathrm{~N}$ & 10.159534102 & -0.410932366 & 3.670337352 \\
$\mathrm{~N}$ & 6.680773640 & 1.011193285 & -1.046926831 \\
$\mathrm{H}$ & 7.592564208 & -1.329975685 & 3.024849516 \\
$\mathrm{H}$ & 6.918528742 & -1.029636502 & 4.652172860 \\
$\mathrm{H}$ & 9.216723249 & -1.798618821 & 4.895558936 \\
$\mathrm{H}$ & 9.108248085 & -0.120146052 & 5.454557008 \\
$\mathrm{H}$ & 6.235758539 & -0.471230799 & 1.032260765 \\
$\mathrm{H}$ & 4.516210473 & -0.162984125 & 0.805038981 \\
$\mathrm{H}$ & 4.901058194 & 1.923779242 & -0.357561169 \\
$\mathrm{H}$ & 6.355505296 & 2.262988417 & 0.580815926 \\
$\mathrm{H}$ & 10.091394621 & -0.953502302 & 2.805226721 \\
$\mathrm{H}$ & 10.113530166 & 0.578555620 & 3.402127802 \\
$\mathrm{H}$ & 6.168838849 & 0.569106390 & -1.821758043 \\
$\mathrm{H}$ & 7.392707472 & 0.338297413 & -0.690472301 \\
$\mathrm{Pd}$ & 8.507126765 & 1.761229834 & 1.695569238 \\
$\mathrm{Pd}$ & 4.141118556 & -0.560413354 & 4.020397943 \\
$\mathrm{Cl}$ & 9.467469194 & 2.639546292 & -0.221015795 \\
$\mathrm{Cl}$ & 8.818426196 & -0.401028300 & 0.784000710 \\
$\mathrm{Cl}$ & 8.098951902 & 3.871968270 & 2.523043668 \\
$\mathrm{Cl}$ & 2.882043818 & -1.956139507 & 5.358631478 \\
$\mathrm{Cl}$ & 3.529175111 & 1.300048090 & 5.275431138 \\
$\mathrm{Cl}$ & 4.883905631 & -2.353173634 & 2.725687389 \\
$\mathrm{Pd}$ & 12.048350721 & -0.621365549 & 4.410419030 \\
$\mathrm{Cl}$ & 14.255357553 & -0.808971245 & 5.107801480 \\
$\mathrm{Cl}$ & 11.750502215 & -2.923196750 & 4.177703747 \\
$\mathrm{Cl}$ & 12.021117939 & 1.716564422 & 4.551067480 \\
$\mathrm{H}$ & 7.238318690 & 1.786159801 & -1.432075510 \\
& & & \\
$\mathrm{C}$ & {$\left[\mathrm{Pd}{ }_{3} \mathrm{Cl}{ }_{9}\left(\mathrm{~S}, \mathrm{~N}, \mathrm{~N}{ }^{\prime}-\mathrm{Cyst}\right)\right]^{3-}$} & \\
$\mathrm{S}$ & 7.775247791 & 2.001843151 & 2.386180062 \\
$\mathrm{~S}$ & 6.310346367 & 0.523218636 & 2.561901751 \\
$\mathrm{C}$ & 9.187505762 & 1.081798250 & 1.682492619 \\
$\mathrm{C}$ & 9.812825891 & 0.078453424 & 2.634332801 \\
& 5.691876241 & 0.271282087 & 0.871204933
\end{tabular}




$\begin{array}{crrr}\mathrm{C} & 4.709259192 & 1.345404362 & 0.443057879 \\ \mathrm{~N} & 10.351518665 & 0.669628458 & 3.860775130 \\ \mathrm{~N} & 4.419713995 & 1.252800752 & -0.991418399 \\ \mathrm{H} & 9.894361287 & 1.885243503 & 1.412430580 \\ \mathrm{H} & 8.863890417 & 0.634088668 & 0.731632520 \\ \mathrm{H} & 10.650358715 & -0.417450998 & 2.119264881 \\ \mathrm{H} & 9.082053992 & -0.694726755 & 2.918116675 \\ \mathrm{H} & 6.541092842 & 0.225670148 & 0.174104875 \\ \mathrm{H} & 5.232394913 & -0.730614954 & 0.870141931 \\ \mathrm{H} & 3.760806954 & 1.263422063 & 0.994623924 \\ \mathrm{H} & 5.151907561 & 2.335312207 & 0.661606617 \\ \mathrm{H} & 9.601095326 & 0.966332159 & 4.490990672 \\ \mathrm{H} & 10.932171127 & 1.478451318 & 3.633558762 \\ \mathrm{H} & 5.309527480 & 1.285759946 & -1.502490409 \\ \mathrm{H} & 3.872271272 & 2.072038729 & -1.263903167 \\ \mathrm{Pd} & 11.574526738 & -0.554226797 & 5.000491185 \\ \mathrm{Pd} & 3.243323700 & -0.324735242 & -1.608689927 \\ \mathrm{Cl} & 12.958382707 & -1.899185505 & 6.276637295 \\ \mathrm{Cl} & 13.149386182 & -0.271406255 & 3.277882200 \\ \mathrm{Cl} & 9.829734301 & -0.572299511 & 6.556451112 \\ \mathrm{Cl} & 1.869027575 & -2.071097870 & -2.286006878 \\ \mathrm{Cl} & 1.446749765 & 1.118503685 & -1.126735935 \\ \mathrm{Cl} & 5.206504642 & -1.525759299 & -2.013168580 \\ \mathrm{Pd} & 7.223892643 & 3.690779872 & 0.852367729 \\ \mathrm{Cl} & 6.759119423 & 4.992291756 & 2.722320136 \\ \mathrm{Cl} & 7.703082499 & 2.321156021 & -0.996686712 \\ \mathrm{Cl} & 6.762720303 & 5.456602461 & -0.559117723\end{array}$

$\left[\mathrm{Pd}_{3} \mathrm{Cl}_{9}(\mathrm{~S}, \mathrm{~N}, \mathrm{~N} \text { '-Cyst })\right]^{3-} \mathrm{SMD}$

$\begin{array}{crrr}\mathrm{S} & 7.802302814 & 2.030312870 & 2.337020213 \\ \mathrm{~S} & 6.212498187 & 0.758683775 & 2.780268441 \\ \mathrm{C} & 9.038824793 & 0.898706357 & 1.621286500 \\ \mathrm{C} & 9.665073782 & -0.031356388 & 2.639011361 \\ \mathrm{C} & 5.508884257 & 0.377521356 & 1.150601925 \\ \mathrm{C} & 4.620197890 & 1.483509147 & 0.605537078 \\ \mathrm{~N} & 10.365484289 & 0.664545146 & 3.721276466 \\ \mathrm{~N} & 4.520276601 & 1.408635762 & -0.854593546 \\ \mathrm{H} & 9.785653137 & 1.579435435 & 1.179144733 \\ \mathrm{H} & 8.586174790 & 0.354033108 & 0.781838308 \\ \mathrm{H} & 10.386090468 & -0.669164749 & 2.105640237 \\ \mathrm{H} & 8.919017663 & -0.697979317 & 3.096295154 \\ \mathrm{H} & 6.319150593 & 0.131217565 & 0.448204231 \\ \mathrm{H} & 4.949142498 & -0.558355681 & 1.311126896 \\ \mathrm{H} & 3.608848276 & 1.428644308 & 1.033304701 \\ \mathrm{H} & 5.041512352 & 2.469056056 & 0.881877331 \\ \mathrm{H} & 9.691708814 & 1.111323498 & 4.346499739 \\ \mathrm{H} & 10.985945860 & 1.388762190 & 3.347328790 \\ \mathrm{H} & 5.466203019 & 1.514064086 & -1.249097010 \\ \mathrm{H} & 3.943991278 & 2.186701446 & -1.182048528 \\ \mathrm{Pd} & 11.598003507 & -0.532072205 & 4.848336253 \\ \mathrm{Pd} & 3.611858324 & -0.265146592 & -1.621354265 \\ \mathrm{Cl} & 13.055847641 & -1.811008228 & 6.121717347\end{array}$




$\begin{array}{lrrr}\mathrm{Cl} & 13.313006856 & 0.284640739 & 3.489569590 \\ \mathrm{Cl} & 9.692482736 & -1.214403827 & 6.019005630 \\ \mathrm{Cl} & 2.522983959 & -2.125027011 & -2.495392004 \\ \mathrm{Cl} & 1.619413707 & 0.908252360 & -1.264404582 \\ \mathrm{Cl} & 5.717247247 & -1.253211396 & -1.833940144 \\ \mathrm{Pd} & 7.219454198 & 3.612176247 & 0.724557953 \\ \mathrm{Cl} & 6.646367232 & 4.977622606 & 2.513637628 \\ \mathrm{Cl} & 7.711434075 & 2.163956561 & -1.049408381 \\ \mathrm{Cl} & 6.751595424 & 5.314503247 & -0.768499507\end{array}$

\begin{tabular}{lccr} 
& \multicolumn{3}{c}{$\left[\mathrm{Pd}_{3} \mathrm{Cl}_{9}\left(\mathrm{~S}, \mathrm{~N}, \mathrm{~N}^{\prime}-\mathrm{H}_{2} \mathrm{CysS}^{3}\right)\right]^{3-}$} \\
$\mathrm{S}$ & 0.484361385 & 0.684162431 & 0.635926475 \\
$\mathrm{~S}$ & -0.240718366 & -1.258572483 & 0.604223120 \\
$\mathrm{O}$ & 2.826875572 & 3.284972161 & 0.794487973 \\
$\mathrm{O}$ & -3.391411264 & -2.852721815 & 1.693282087 \\
$\mathrm{O}$ & 1.877018794 & 3.868177123 & -1.139439830 \\
$\mathrm{O}$ & -2.056974001 & -3.799498073 & 0.151596302 \\
$\mathrm{~N}$ & 3.623406936 & 0.853969684 & -0.284253160 \\
$\mathrm{~N}$ & -3.486290901 & -0.487093565 & 0.359135382 \\
$\mathrm{C}$ & 2.489120390 & 2.997761151 & -0.322355639 \\
$\mathrm{C}$ & -2.810845880 & -2.824756199 & 0.637832265 \\
$\mathrm{C}$ & 2.594904288 & 1.630013026 & -0.954978942 \\
$\mathrm{C}$ & -2.839296029 & -1.619860592 & -0.298985547 \\
$\mathrm{C}$ & 1.215733559 & 0.965239555 & -1.018527527 \\
$\mathrm{C}$ & -1.439311892 & -1.282649426 & -0.797278335 \\
$\mathrm{H}$ & 3.468946953 & 0.813271630 & 0.728857957 \\
$\mathrm{H}$ & -4.032533865 & -0.822472502 & 1.161622362 \\
$\mathrm{H}$ & 3.596323655 & -0.115230786 & -0.623083998 \\
$\mathrm{H}$ & -2.781246650 & 0.131756800 & 0.765765198 \\
$\mathrm{H}$ & 2.931661824 & 1.799973495 & -1.992226701 \\
$\mathrm{H}$ & -3.424978166 & -1.870083503 & -1.200618432 \\
$\mathrm{H}$ & 1.814480304 & 4.693661601 & -0.637533986 \\
$\mathrm{H}$ & -1.568905140 & -4.242563770 & 0.898399766 \\
$\mathrm{H}$ & 1.321431906 & -0.020225438 & -1.500621931 \\
$\mathrm{H}$ & -1.461364479 & -0.306409572 & -1.302645210 \\
$\mathrm{H}$ & 0.523595025 & 1.595718170 & -1.595637221 \\
$\mathrm{H}$ & -1.076733588 & -2.039052870 & -1.503772840 \\
$\mathrm{Pd}$ & 1.218005697 & -3.077973868 & 0.278767819 \\
$\mathrm{Pd}$ & -4.880214336 & 0.718025828 & -0.640248235 \\
$\mathrm{Cl}$ & 2.436996516 & -5.006445430 & -0.017336293 \\
$\mathrm{Cl}$ & -6.469047597 & 2.048852187 & -1.655814250 \\
$\mathrm{Cl}$ & 0.114771628 & -4.076352859 & 2.119668188 \\
$\mathrm{Cl}$ & -5.842857003 & 0.946697431 & 1.484253333 \\
$\mathrm{Cl}$ & 2.246872983 & -2.147984358 & -1.596565795 \\
$\mathrm{Cl}$ & 5.821638355 & 0.578699069 & 1.709983901 \\
$\mathrm{Cl}$ & -3.771635776 & 0.307308838 & -2.664612378 \\
$\mathrm{Pd}$ & 5.604830846 & 1.577993884 & -0.389647932 \\
& 7.792481446 & 2.332543297 & -0.399596708 \\
& 0.095507166 & 1.196880803 & 0.293765659
\end{tabular}




$\begin{array}{lrrr}\mathrm{S} & -0.173766347 & -0.852965489 & 0.459233878 \\ \mathrm{O} & 2.973734542 & 3.236307314 & 0.738504730 \\ \mathrm{O} & -2.803918572 & -2.808151661 & 1.974191888 \\ \mathrm{O} & 2.284833218 & 4.133939787 & -1.200898274 \\ \mathrm{O} & -1.504816634 & -3.734523594 & 0.394306140 \\ \mathrm{~N} & 3.135301639 & 0.744772838 & -0.388174375 \\ \mathrm{~N} & -3.447789930 & -0.693745386 & 0.419642153 \\ \mathrm{C} & 2.633001817 & 3.118692157 & -0.412969791 \\ \mathrm{C} & -2.358786214 & -2.831570609 & 0.852614830 \\ \mathrm{C} & 2.444607924 & 1.791223076 & -1.118456337 \\ \mathrm{C} & -2.682896628 & -1.773952631 & -0.195449589 \\ \mathrm{C} & 0.952651950 & 1.485694065 & -1.292003059 \\ \mathrm{C} & -1.413129851 & -1.244699873 & -0.846140096 \\ \mathrm{H} & 2.743844553 & 0.667069391 & 0.556418286 \\ \mathrm{H} & -3.991661504 & -1.067433634 & 1.203228569 \\ \mathrm{H} & 2.943369350 & -0.158116780 & -0.845265734 \\ \mathrm{H} & -2.806903339 & -0.039827864 & 0.879612468 \\ \mathrm{H} & 2.886557604 & 1.889364615 & -2.121034762 \\ \mathrm{H} & -3.286572667 & -2.232765649 & -0.995633992 \\ \mathrm{H} & 2.331467874 & 4.975159774 & -0.711696812 \\ \mathrm{H} & -0.976486834 & -4.093675517 & 1.145145594 \\ \mathrm{H} & 0.828306978 & 0.592952634 & -1.922325904 \\ \mathrm{H} & -1.642600328 & -0.342114016 & -1.429665058 \\ \mathrm{H} & 0.435697950 & 2.328310761 & -1.775145276 \\ \mathrm{H} & -0.947855720 & -1.983085887 & -1.508547105 \\ \mathrm{Pd} & 1.488720480 & -2.479598720 & 0.097817127 \\ \mathrm{Pd} & -4.829074404 & 0.451389453 & -0.633213799 \\ \mathrm{Cl} & 2.803113152 & -4.328942432 & -0.280000682 \\ \mathrm{Cl} & -6.453892732 & 1.719966298 & -1.679693932 \\ \mathrm{Cl} & 0.892730136 & -3.334653571 & 2.193162470 \\ \mathrm{Cl} & -5.545139506 & 1.067826112 & 1.499695102 \\ \mathrm{Cl} & 1.905874700 & -1.722183215 & -2.080452044 \\ \mathrm{Cl} & -3.977977505 & -0.269927195 & -2.691095575 \\ \mathrm{Pd} & 5.162523422 & 0.784836174 & -0.024038114 \\ \mathrm{Cl} & 7.414460436 & 0.757378989 & 0.528605109 \\ \mathrm{Cl} & 5.499734883 & 2.404852776 & -1.679939298 \\ \mathrm{Cl} & 4.603903346 & -0.811078932 & 1.595800613\end{array}$

$\left[\mathrm{Pd}_{3} \mathrm{Cl}_{9}\left(\mathrm{~S}, \mathrm{~S}^{\prime}, \mathrm{N}-\mathrm{H}_{3} \mathrm{CysS}\right)\right]^{2-}$

$\begin{array}{rrrr}\text { S } & 0.494553955 & 0.908108795 & 0.684504762 \\ \text { S } & -0.306457978 & -1.069642033 & 0.698825932 \\ \mathrm{O} & 3.237227186 & 3.301113991 & 1.064779890 \\ \mathrm{O} & -3.729752931 & -2.462221881 & 1.122690937 \\ \mathrm{O} & 1.746600819 & 3.890752971 & -0.494282860 \\ \mathrm{O} & -2.081497602 & -3.615912898 & 0.097965517 \\ \mathrm{~N} & 3.739267166 & 0.893007317 & -0.160617472 \\ \mathrm{~N} & -3.338085236 & -0.290161152 & -0.286151344 \\ \mathrm{C} & 2.635490670 & 3.047627821 & 0.059136894 \\ \mathrm{C} & -2.882743175 & -2.600380877 & 0.276137102 \\ \mathrm{C} & 2.725378316 & 1.761243550 & -0.726911327 \\ \mathrm{C} & -2.614642270 & -1.489077355 & -0.749628271 \\ \mathrm{C} & 1.354639053 & 1.121586436 & -0.923621136\end{array}$




$\begin{array}{lrrr}\mathrm{C} & -1.128783022 & -1.210009816 & -0.935531370 \\ \mathrm{H} & 3.798002096 & 0.951381643 & 0.861994187 \\ \mathrm{H} & -2.912326520 & 0.085202134 & 0.587764138 \\ \mathrm{H} & 3.566669533 & -0.086520213 & -0.411093079 \\ \mathrm{H} & -3.242979316 & 0.518842641 & -0.949477898 \\ \mathrm{H} & 3.077092646 & 2.041245485 & -1.748777487 \\ \mathrm{H} & -3.033540962 & -1.786317084 & -1.726061655 \\ \mathrm{H} & 1.703825195 & 4.661908537 & 0.090106163 \\ \mathrm{H} & -1.710274837 & -3.960550923 & 0.979708578 \\ \mathrm{H} & 1.477705935 & 0.122233702 & -1.370685778 \\ \mathrm{H} & -0.985022838 & -0.280587090 & -1.508153525 \\ \mathrm{H} & 0.700341248 & 1.750530857 & -1.541439633 \\ \mathrm{H} & -0.637878309 & -2.040863758 & -1.457855718 \\ \mathrm{Pd} & 1.089181430 & -2.935438322 & 0.723454847 \\ \mathrm{Pd} & -1.568278897 & 2.016030872 & 0.694212873 \\ \mathrm{Cl} & 2.196890637 & -4.926222980 & 0.660756484 \\ \mathrm{Cl} & -3.797237275 & 2.643471102 & 0.870348634 \\ \mathrm{Cl} & -0.361722755 & -3.775907715 & 2.404952663 \\ \mathrm{Cl} & -1.360569460 & 1.936722903 & 2.970450342 \\ \mathrm{Cl} & 2.274360670 & -2.156080298 & -1.116597077 \\ \mathrm{Cl} & -1.849109705 & 1.997491775 & -1.672925026 \\ \mathrm{Pd} & 5.749578743 & 1.282127310 & -0.679055640 \\ \mathrm{Cl} & 7.964838103 & 1.669686094 & -1.162486236 \\ \mathrm{Cl} & 5.057245894 & 1.977295794 & -2.824764123 \\ \mathrm{Cl} & 6.189599126 & 0.576696210 & 1.497869033 \\ \mathrm{H} & -4.311458931 & -0.494470039 & -0.041899547\end{array}$

$\left[\mathrm{Pd}_{3} \mathrm{Cl}_{9}\left(\mathrm{~S}, \mathrm{~S}^{\prime}, \mathrm{N}-\mathrm{H}_{3} \mathrm{CysS}\right)\right]^{2-} \mathrm{SMD}$

$\begin{array}{lrrr}\mathrm{S} & 0.356184111 & 1.017118254 & 0.685665369 \\ \mathrm{~S} & -0.314430485 & -1.008774279 & 0.649696917 \\ \mathrm{O} & 3.111107159 & 3.024453117 & 1.085901921 \\ \mathrm{O} & -3.914165698 & -2.902064099 & 0.719145304 \\ \mathrm{O} & 2.393803455 & 3.949272855 & -0.834361000 \\ \mathrm{O} & -1.947267739 & -3.647943067 & -0.086791254 \\ \mathrm{~N} & 3.451560836 & 0.592714698 & -0.188092883 \\ \mathrm{~N} & -3.454968703 & -0.417726493 & -0.149120301 \\ \mathrm{C} & 2.766019967 & 2.929312211 & -0.065178174 \\ \mathrm{C} & -2.918273429 & -2.767273696 & 0.055535524 \\ \mathrm{C} & 2.626533160 & 1.618052350 & -0.809011195 \\ \mathrm{C} & -2.659977904 & -1.508967373 & -0.765750937 \\ \mathrm{C} & 1.155607776 & 1.232797603 & -0.947263269 \\ \mathrm{C} & -1.199816802 & -1.140629016 & -0.948640486 \\ \mathrm{H} & 3.299343063 & 0.541545577 & 0.825602247 \\ \mathrm{H} & -3.008946736 & -0.058236697 & 0.711448366 \\ \mathrm{H} & 3.226482970 & -0.323974612 & -0.594743098 \\ \mathrm{H} & -3.536194310 & 0.397420706 & -0.785383169 \\ \mathrm{H} & 3.004402780 & 1.766365006 & -1.834297550 \\ \mathrm{H} & -3.082060549 & -1.700103233 & -1.769670401 \\ \mathrm{H} & 2.400395321 & 4.784827548 & -0.331616905 \\ \mathrm{H} & -2.118703906 & -4.471486381 & 0.411051171 \\ \mathrm{H} & 1.094511881 & 0.280467331 & -1.498260973 \\ \mathrm{H} & -1.123481586 & -0.193793202 & -1.504481284\end{array}$




$\begin{array}{lrrr}\mathrm{H} & 0.579446491 & 1.994442255 & -1.490649849 \\ \mathrm{H} & -0.673724710 & -1.916251667 & -1.519341128 \\ \mathrm{Pd} & 1.665001650 & -2.204662781 & 0.629953927 \\ \mathrm{Pd} & -1.682777831 & 2.094439547 & 0.847420265 \\ \mathrm{Cl} & 3.674202570 & -3.316168849 & 0.742530571 \\ \mathrm{Cl} & -3.818488464 & 2.894033071 & 1.157963222 \\ \mathrm{Cl} & 1.364036845 & -2.349184612 & 2.921018842 \\ \mathrm{Cl} & -1.330374495 & 1.987661693 & 3.122031728 \\ \mathrm{Cl} & 1.888089883 & -2.026501116 & -1.697499559 \\ \mathrm{Cl} & -2.035955819 & 2.112728346 & -1.474191533 \\ \mathrm{Pd} & 5.500893321 & 0.819390214 & -0.349006579 \\ \mathrm{Cl} & 7.799623543 & 1.091345289 & -0.496131276 \\ \mathrm{Cl} & 5.218939603 & 1.115839716 & -2.651948169 \\ \mathrm{Cl} & 5.554256482 & 0.449300736 & 1.951600858 \\ \mathrm{H} & -4.384707299 & -0.765843445 & 0.132507511\end{array}$

\begin{tabular}{lrrr} 
& \multicolumn{4}{c}{$\left[\mathrm{Pd}_{4} \mathrm{Cl}_{12}\left(\mathrm{~S}^{\prime} \mathrm{S}^{\prime}, \mathrm{N}^{\prime} \mathrm{N}^{\prime}-\mathrm{HCyst}\right)\right]^{4-}$} \\
$\mathrm{S}$ & -0.101686943 & 1.062120143 & 1.126120155 \\
$\mathrm{~S}$ & 0.101790156 & -1.062234235 & 1.126027115 \\
$\mathrm{C}$ & 0.753713475 & 1.527956706 & -0.419867772 \\
$\mathrm{C}$ & -0.753594219 & -1.527981489 & -0.419944153 \\
$\mathrm{C}$ & 1.163416466 & 2.996275893 & -0.429957274 \\
$\mathrm{C}$ & -1.163444221 & -2.996295072 & -0.429961660 \\
$\mathrm{~N}$ & 0.071022004 & 3.974113329 & -0.456837141 \\
$\mathrm{~N}$ & -0.071059024 & -3.974134926 & -0.456970934 \\
$\mathrm{H}$ & 0.078291470 & 1.304668697 & -1.258224303 \\
$\mathrm{H}$ & -0.078199787 & -1.304711366 & -1.258313905 \\
$\mathrm{H}$ & 1.648452045 & 0.887402098 & -0.481354840 \\
$\mathrm{H}$ & -1.648328640 & -0.887422572 & -0.481375803 \\
$\mathrm{H}$ & 1.746845427 & 3.151459660 & -1.351416191 \\
$\mathrm{H}$ & -1.746809368 & -3.151390366 & -1.351486905 \\
$\mathrm{H}$ & 1.817493462 & 3.233084159 & 0.423532117 \\
$\mathrm{H}$ & -1.817583961 & -3.233099746 & 0.423454997 \\
$\mathrm{H}$ & -0.674860064 & 3.649325627 & -1.084943024 \\
$\mathrm{H}$ & 0.674823938 & -3.649346849 & -1.085050543 \\
$\mathrm{H}$ & -0.352183052 & 4.103267685 & 0.467090016 \\
$\mathrm{H}$ & 0.352048295 & -4.103245072 & 0.467077776 \\
$\mathrm{Pd}$ & -2.397792662 & 1.456362130 & 0.963013144 \\
$\mathrm{Pd}$ & 2.397848395 & -1.456486414 & 0.963017235 \\
$\mathrm{Cl}$ & -4.675271519 & 1.883695756 & 0.978406868 \\
$\mathrm{Cl}$ & 4.675423768 & -1.883388258 & 0.978630179 \\
$\mathrm{Cl}$ & -2.344221895 & 1.788331872 & -1.358199190 \\
$\mathrm{Cl}$ & 2.344250975 & -1.788345533 & -1.358209482 \\
$\mathrm{Cl}$ & -2.394536382 & 1.085603889 & 3.257159854 \\
$\mathrm{Cl}$ & 2.394658225 & -1.085573764 & 3.257193837 \\
$\mathrm{Pd}$ & 0.652259678 & 5.890307654 & -1.015667882 \\
$\mathrm{Pd}$ & -0.652303456 & -5.890311942 & -1.015678269 \\
$\mathrm{Cl}$ & 1.306319169 & 8.045824550 & -1.626110487 \\
$\mathrm{Cl}$ & -1.306438263 & -8.045848032 & -1.625988257 \\
$\mathrm{Cl}$ & 1.063285737 & 5.028158923 & -3.156103100 \\
$\mathrm{Cl}$ & -1.063345925 & -5.028272012 & -3.156150348 \\
$\mathrm{Cl}$ & 0.208489203 & 6.504238298 & 1.203270931
\end{tabular}




\begin{tabular}{lrrr} 
& \multicolumn{3}{c}{$\left[\mathrm{Pd}_{4} \mathrm{Cl}_{12}\left(\mathrm{~S}, \mathrm{~S}^{\prime}, \mathrm{N}, \mathrm{N}^{\prime}-\mathrm{HCyst}\right)\right]^{4-} \mathrm{SMD}$} \\
$\mathrm{S}$ & 0.053377050 & 1.062011867 & 1.069909699 \\
$\mathrm{~S}$ & -0.077664624 & -1.049843518 & 1.082580098 \\
$\mathrm{C}$ & 0.889302493 & 1.423687138 & -0.510975299 \\
$\mathrm{C}$ & -0.893400669 & -1.436942857 & -0.502007494 \\
$\mathrm{C}$ & 1.243269592 & 2.902955239 & -0.576788718 \\
$\mathrm{C}$ & -1.250784480 & -2.914797402 & -0.555327224 \\
$\mathrm{~N}$ & 0.084439842 & 3.795423903 & -0.492588513 \\
$\mathrm{~N}$ & -0.092261159 & -3.809789767 & -0.490463747 \\
$\mathrm{H}$ & 0.214145445 & 1.145271139 & -1.332102862 \\
$\mathrm{H}$ & -0.209800311 & -1.169860118 & -1.319982999 \\
$\mathrm{H}$ & 1.796696413 & 0.801792874 & -0.549965051 \\
$\mathrm{H}$ & -1.800383496 & -0.816501546 & -0.561513281 \\
$\mathrm{H}$ & 1.734113793 & 3.076113320 & -1.546856987 \\
$\mathrm{H}$ & -1.754406956 & -3.086783869 & -1.518956620 \\
$\mathrm{H}$ & 1.961118185 & 3.178261559 & 0.210475358 \\
$\mathrm{H}$ & -1.960225022 & -3.186318116 & 0.241229458 \\
$\mathrm{H}$ & -0.668504111 & 3.435868387 & -1.093110461 \\
$\mathrm{H}$ & 0.663303008 & -3.429282425 & -1.075414195 \\
$\mathrm{H}$ & -0.287000593 & 3.827326618 & 0.462839186 \\
$\mathrm{H}$ & 0.285449543 & -3.861160157 & 0.461533138 \\
$\mathrm{Pd}$ & -2.169494845 & 1.759805709 & 1.006206350 \\
$\mathrm{Pd}$ & 2.151304369 & -1.724348354 & 1.062092552 \\
$\mathrm{Cl}$ & -4.373567984 & 2.440581036 & 1.098733439 \\
$\mathrm{Cl}$ & 4.348868929 & -2.416462108 & 1.203121483 \\
$\mathrm{Cl}$ & -2.286468410 & 1.741199705 & -1.337226237 \\
$\mathrm{Cl}$ & 2.297367613 & -1.782867792 & -1.279484617 \\
$\mathrm{Cl}$ & -1.995802138 & 1.745961427 & 3.317976807 \\
$\mathrm{Cl}$ & 1.971916252 & -1.512759279 & 3.365910802 \\
$\mathrm{Pd}$ & 0.442010401 & 5.762474757 & -0.909722294 \\
$\mathrm{Pd}$ & -0.415164621 & -5.778983956 & -0.948400780 \\
$\mathrm{Cl}$ & 0.891578082 & 7.996187224 & -1.362538703 \\
$\mathrm{Cl}$ & -0.765738999 & -8.013383747 & -1.450195734 \\
$\mathrm{Cl}$ & 0.752313735 & 5.095119488 & -3.121563368 \\
$\mathrm{Cl}$ & -1.026426841 & -5.090658817 & -3.093840850 \\
$\mathrm{Cl}$ & -0.022326390 & 6.124711166 & 1.359730260 \\
$\mathrm{Cl}$ & 0.268846903 & -6.234008729 & 1.246218038
\end{tabular}

\begin{tabular}{lrrr} 
& \multicolumn{4}{c}{$\left[\mathrm{Pd}_{4} \mathrm{Cl}_{12}\left(\mathrm{~S}, \mathrm{~S}^{\prime}, \mathrm{N}^{\prime} \mathrm{N}^{\prime}-\mathrm{H}_{2} \mathrm{CysS}\right)\right]^{4-}$} \\
$\mathrm{S}$ & 0.404683758 & 0.972012796 & 0.650529235 \\
$\mathrm{~S}$ & -0.404683758 & -0.972012796 & 0.650529235 \\
$\mathrm{O}$ & 3.363202439 & 2.675771290 & 1.543692353 \\
$\mathrm{O}$ & -3.363202439 & -2.675771290 & 1.543692353 \\
$\mathrm{O}$ & 1.810312673 & 3.728517627 & 0.310137080 \\
$\mathrm{O}$ & -1.810312673 & -3.728517627 & 0.310137080 \\
$\mathrm{~N}$ & 3.822051340 & 0.812722958 & -0.384973403 \\
$\mathrm{~N}$ & -3.822051340 & -0.812722958 & -0.384973403 \\
$\mathrm{C}$ & 2.721891454 & 2.781456941 & 0.532972468 \\
$\mathrm{C}$ & -2.721891454 & -2.781456941 & 0.532972468 \\
$\mathrm{C}$ & 2.770305550 & 1.797170362 & -0.623119213
\end{tabular}




$\begin{array}{lrrr}\mathrm{C} & -2.770305550 & -1.797170362 & -0.623119213 \\ \mathrm{C} & 1.371487024 & 1.215673572 & -0.895414494 \\ \mathrm{C} & -1.371487024 & -1.215673572 & -0.895414494 \\ \mathrm{H} & 3.930669133 & 0.622827485 & 0.617288679 \\ \mathrm{H} & -3.930669133 & -0.622827485 & 0.617288679 \\ \mathrm{H} & 3.593165649 & -0.080374002 & -0.836015689 \\ \mathrm{H} & -3.593165649 & 0.080374002 & -0.836015689 \\ \mathrm{H} & 3.052008209 & 2.353795075 & -1.537531202 \\ \mathrm{H} & -3.052008209 & -2.353795075 & -1.537531202 \\ \mathrm{H} & 1.423846860 & 4.006237592 & 1.175840067 \\ \mathrm{H} & -1.423846860 & -4.006237592 & 1.175840067 \\ \mathrm{H} & 1.456106556 & 0.232963781 & -1.386278626 \\ \mathrm{H} & -1.456106556 & -0.232963781 & -1.386278626 \\ \mathrm{H} & 0.764756712 & 1.887272095 & -1.515828138 \\ \mathrm{H} & -0.764756712 & -1.887272095 & -1.515828138 \\ \mathrm{Pd} & 1.220737228 & -2.674386372 & 0.639210973 \\ \mathrm{Pd} & -1.220737228 & 2.674386372 & 0.639210973 \\ \mathrm{Cl} & 2.631212282 & -4.499102882 & 0.661365244 \\ \mathrm{Cl} & -2.631212282 & 4.499102882 & 0.661365244 \\ \mathrm{Cl} & 0.171323479 & -3.467133384 & 2.593768385 \\ \mathrm{Cl} & -0.171323479 & 3.467133384 & 2.593768385 \\ \mathrm{Cl} & 2.093524703 & -2.023948030 & -1.434373535 \\ \mathrm{Cl} & -2.093524703 & 2.023948030 & -1.434373535 \\ \mathrm{Pd} & 5.802188685 & 1.331426569 & -0.906974862 \\ \mathrm{Pd} & -5.802188685 & -1.331426569 & -0.906974862 \\ \mathrm{Cl} & 8.010991749 & 1.859380615 & -1.419296789 \\ \mathrm{Cl} & -8.010991749 & -1.859380615 & -1.419296789 \\ \mathrm{Cl} & 5.030201810 & 2.375678243 & -2.873358895 \\ \mathrm{Cl} & -5.030201810 & -2.375678243 & -2.873358895 \\ \mathrm{Cl} & 6.412609459 & 0.236101753 & 1.062238406 \\ \mathrm{Cl} & -6.412609459 & -0.236101753 & 1.062238406\end{array}$

\begin{tabular}{lrrr} 
& \multicolumn{4}{c}{$\left[\mathrm{Pd}_{4} \mathrm{Cl}_{12}\left(\mathrm{~S}, \mathrm{~S}^{\prime}, \mathrm{N}, \mathrm{N}^{\prime}-\mathrm{H}_{2} \mathrm{CysS}\right)\right]^{4-} \mathrm{SMD}$} \\
$\mathrm{S}$ & 0.270293009 & 1.000259073 & 0.632888664 \\
$\mathrm{~S}$ & -0.337574416 & -1.039567073 & 0.612355552 \\
$\mathrm{O}$ & 3.120100838 & 2.999598693 & 1.193151955 \\
$\mathrm{O}$ & -3.317670373 & -3.181335003 & 0.877520649 \\
$\mathrm{O}$ & 2.174660998 & 3.948531043 & -0.610510952 \\
$\mathrm{O}$ & -2.135089567 & -3.941364256 & -0.873778794 \\
$\mathrm{~N}$ & 3.449279843 & 0.620565293 & -0.126763410 \\
$\mathrm{~N}$ & -3.431497913 & -0.649631757 & -0.157541271 \\
$\mathrm{C}$ & 2.690511606 & 2.925133740 & 0.069835239 \\
$\mathrm{C}$ & -2.750668675 & -2.992470919 & -0.168230658 \\
$\mathrm{C}$ & 2.613156575 & 1.645754281 & -0.734090727 \\
$\mathrm{C}$ & -2.618298901 & -1.644277523 & -0.843497265 \\
$\mathrm{C}$ & 1.165573502 & 1.216345370 & -0.952222928 \\
$\mathrm{C}$ & -1.157226509 & -1.240756065 & -1.010215145 \\
$\mathrm{H}$ & 3.293408608 & 0.555564342 & 0.884835288 \\
$\mathrm{H}$ & -3.187836985 & -0.578824030 & 0.837264570 \\
$\mathrm{H}$ & 3.230630147 & -0.293563875 & -0.543960086 \\
$\mathrm{H}$ & -3.260567023 & 0.269501543 & -0.586309744 \\
$\mathrm{H}$ & 3.026468410 & 1.851437880 & -1.736909840
\end{tabular}




$\begin{array}{lrrr}\mathrm{H} & -3.029725891 & -1.762290022 & -1.861845919 \\ \mathrm{H} & 2.160505934 & 4.753511737 & -0.060010875 \\ \mathrm{H} & -2.213204790 & -4.800229370 & -0.419095030 \\ \mathrm{H} & 1.154676020 & 0.259084131 & -1.496392867 \\ \mathrm{H} & -1.108934100 & -0.282070102 & -1.549445915 \\ \mathrm{H} & 0.602470378 & 1.958195380 & -1.533894245 \\ \mathrm{H} & -0.577593554 & -1.990669325 & -1.563929602 \\ \mathrm{Pd} & 1.674001626 & -2.181820257 & 0.719657509 \\ \mathrm{Pd} & -1.673560513 & 2.253259947 & 0.588230024 \\ \mathrm{Cl} & 3.721199979 & -3.200633646 & 0.988237752 \\ \mathrm{Cl} & -3.559428794 & 3.567365761 & 0.635348575 \\ \mathrm{Cl} & 1.268140449 & -2.298468987 & 2.992997492 \\ \mathrm{Cl} & -1.323715442 & 2.547655891 & 2.855474131 \\ \mathrm{Cl} & 2.026499998 & -2.063060243 & -1.593817316 \\ \mathrm{Cl} & -1.993064684 & 1.966383946 & -1.720187693 \\ \mathrm{Pd} & 5.495714335 & 0.858468586 & -0.285965319 \\ \mathrm{Pd} & -5.482255057 & -0.901632098 & -0.137081885 \\ \mathrm{Cl} & 7.794853106 & 1.133991811 & -0.440243737 \\ \mathrm{Cl} & -7.783580927 & -1.167024265 & -0.069251579 \\ \mathrm{Cl} & 5.207644825 & 1.161376981 & -2.588220199 \\ \mathrm{Cl} & -5.409050115 & -1.385259441 & -2.428114389 \\ \mathrm{Cl} & 5.563737289 & 0.483228613 & 2.012790703 \\ \mathrm{Cl} & -5.352983246 & -0.380265784 & 2.138695378\end{array}$

$\left[\mathrm{Pd}_{2} \mathrm{Cl}_{4}(\mathrm{~S}, \mathrm{~N}-\mathrm{Cyst})\right]$

$\begin{array}{lrrr}\mathrm{S} & -0.797034995 & 0.710850012 & -0.819704860 \\ \mathrm{~S} & 0.797034995 & -0.710850012 & -0.819704860 \\ \mathrm{C} & -1.746572639 & 0.125578341 & 0.629922102 \\ \mathrm{C} & 1.746572639 & -0.125578341 & 0.629922102 \\ \mathrm{C} & -2.728502829 & -0.982760873 & 0.291279341 \\ \mathrm{C} & 2.728502829 & 0.982760873 & 0.291279341 \\ \mathrm{~N} & 2.165344473 & 2.055145641 & -0.541543414 \\ \mathrm{~N} & -2.165344473 & -2.055145641 & -0.541543414 \\ \mathrm{H} & -2.266992940 & 1.036545272 & 0.968517853 \\ \mathrm{H} & 2.266992940 & -1.036545272 & 0.968517853 \\ \mathrm{H} & -1.025148227 & -0.171469103 & 1.405754301 \\ \mathrm{H} & 1.025148227 & 0.171469103 & 1.405754301 \\ \mathrm{H} & -3.616905363 & -0.554253631 & -0.200931139 \\ \mathrm{H} & 3.616905363 & 0.554253631 & -0.200931139 \\ \mathrm{H} & -3.058310118 & -1.430439484 & 1.240467199 \\ \mathrm{H} & 3.058310118 & 1.430439484 & 1.240467199 \\ \mathrm{H} & 2.248311078 & 1.820505061 & -1.532374255 \\ \mathrm{H} & -2.248311078 & -1.820505061 & -1.532374255 \\ \mathrm{H} & 2.667986005 & 2.939760935 & -0.397199459 \\ \mathrm{H} & -2.667986005 & -2.939760935 & -0.397199459 \\ \mathrm{Pd} & 0.218541858 & 2.699556374 & -0.197612094 \\ \mathrm{Pd} & -0.218541858 & -2.699556374 & -0.197612094 \\ \mathrm{Cl} & 1.200255588 & 4.681671319 & 0.380251465 \\ \mathrm{Cl} & -1.200255588 & -4.681671319 & 0.380251465 \\ \mathrm{Cl} & -1.939323198 & 3.410748805 & 0.139265464 \\ \mathrm{Cl} & 1.939323198 & -3.410748805 & 0.139265464\end{array}$


$\left[\mathrm{Pd}_{2} \mathrm{Cl}_{4}(\mathrm{~S}, \mathrm{~N}-\mathrm{Cyst})\right] \mathrm{SMD}$

$\begin{array}{rrrr}\text { S } & -0.778313987 & 0.701697992 & -0.812664822 \\ \mathrm{~S} & 0.791575599 & -0.729671920 & -0.826317907 \\ \mathrm{C} & -1.716775028 & 0.119579915 & 0.644820463 \\ \mathrm{C} & 1.755364814 & -0.143107138 & 0.609575468 \\ \mathrm{C} & -2.719631920 & -0.957339305 & 0.288609280 \\ \mathrm{C} & 2.725624394 & 0.972533316 & 0.274307138 \\ \mathrm{~N} & 2.161503986 & 2.087138065 & -0.512827925 \\ \mathrm{~N} & -2.169517429 & -2.063159954 & -0.518053868 \\ \mathrm{H} & -2.226946847 & 1.024354552 & 1.006809566 \\ \mathrm{H} & 2.300856617 & -1.039518778 & 0.942037689 \\ \mathrm{H} & -1.000001270 & -0.194069703 & 1.419313893 \\ \mathrm{H} & 1.046521642 & 0.129613478 & 1.405827182 \\ \mathrm{H} & -3.576996489 & -0.513329561 & -0.240575682 \\ \mathrm{H} & 3.605674854 & 0.565489540 & -0.247911232 \\ \mathrm{H} & -3.097514590 & -1.373619736 & 1.234184734 \\ \mathrm{H} & 3.081909348 & 1.374253649 & 1.234915881 \\ \mathrm{H} & 2.289137228 & 1.919674519 & -1.517629294 \\ \mathrm{H} & -2.236549804 & -1.841957981 & -1.517374877 \\ \mathrm{H} & 2.676967890 & 2.948261677 & -0.312616909 \\ \mathrm{H} & -2.742367939 & -2.900656582 & -0.377886410 \\ \mathrm{Pd} & 0.206957208 & 2.707044908 & -0.208804444 \\ \mathrm{Pd} & -0.235281948 & -2.722634556 & -0.178343164 \\ \mathrm{Cl} & 1.104705090 & 4.750943539 & 0.379564228 \\ \mathrm{Cl} & -1.180183188 & -4.734516613 & 0.403150445 \\ \mathrm{Cl} & -1.990497791 & 3.400566585 & 0.033161942 \\ \mathrm{Cl} & 1.923779559 & -3.487569907 & 0.126913635\end{array}$

\begin{tabular}{lrrr} 
& \multicolumn{4}{c}{$\left[\mathrm{Pd}_{2} \mathrm{Cl}_{4}\left(\mathrm{~S}, \mathrm{~N}-\mathrm{H}_{2} \mathrm{CysS}\right)\right]$} \\
$\mathrm{S}$ & 0.808194462 & 0.691542158 & 0.129444362 \\
$\mathrm{~S}$ & -0.808194462 & -0.691542158 & 0.129444362 \\
$\mathrm{O}$ & -4.017733529 & 0.729664263 & 1.099154569 \\
$\mathrm{O}$ & 4.017733529 & -0.729664263 & 1.099154569 \\
$\mathrm{O}$ & -4.557958449 & -0.473661465 & -0.716786425 \\
$\mathrm{O}$ & 4.557958449 & 0.473661465 & -0.716786425 \\
$\mathrm{~N}$ & -2.136959292 & 2.148062829 & -0.210123144 \\
$\mathrm{~N}$ & 2.136959292 & -2.148062829 & -0.210123144 \\
$\mathrm{C}$ & -3.852360627 & 0.424582293 & -0.051281121 \\
$\mathrm{C}$ & 3.852360627 & -0.424582293 & -0.051281121 \\
$\mathrm{C}$ & -2.743735824 & 1.018956619 & -0.917410834 \\
$\mathrm{C}$ & 2.743735824 & -1.018956619 & -0.917410834 \\
$\mathrm{C}$ & -1.752269351 & -0.072847794 & -1.311360383 \\
$\mathrm{C}$ & 1.752269351 & 0.072847794 & -1.311360383 \\
$\mathrm{H}$ & -2.319783666 & 2.017910707 & 0.793234283 \\
$\mathrm{H}$ & 2.319783666 & -2.017910707 & 0.793234283 \\
$\mathrm{H}$ & -2.588720963 & 3.034528479 & -0.467692681 \\
$\mathrm{H}$ & 2.588720963 & -3.034528479 & -0.467692681 \\
$\mathrm{H}$ & -3.196106383 & 1.374935116 & -1.857200406 \\
$\mathrm{H}$ & 3.196106383 & -1.374935116 & -1.857200406 \\
$\mathrm{H}$ & -5.185160973 & -0.895059816 & -0.107184445 \\
$\mathrm{H}$ & 5.185160973 & 0.895059816 & -0.107184445 \\
$\mathrm{H}$ & -1.049626263 & 0.285849114 & -2.076928999
\end{tabular}




$\begin{array}{lrrr}\mathrm{H} & 1.049626263 & -0.285849114 & -2.076928999 \\ \mathrm{H} & 2.275858022 & 0.967241848 & -1.685942809 \\ \mathrm{H} & -2.275858022 & -0.967241848 & -1.685942809 \\ \mathrm{Pd} & -0.145475771 & 2.688424666 & -0.519646251 \\ \mathrm{Pd} & 0.145475771 & -2.688424666 & -0.519646251 \\ \mathrm{Cl} & 2.051418233 & 3.313234579 & -0.790693798 \\ \mathrm{Cl} & -2.051418233 & -3.313234579 & -0.790693798 \\ \mathrm{Cl} & -1.026043659 & 4.697566746 & -1.167780529 \\ \mathrm{Cl} & 1.026043659 & -4.697566746 & -1.167780529\end{array}$

\begin{tabular}{lrrr} 
& \multicolumn{3}{c}{$\left[\mathrm{Pd}_{2} \mathrm{Cl}_{4}\left(\mathrm{~S}, \mathrm{~N}-\mathrm{H}_{2} \mathrm{CySS}\right)\right] \mathrm{SMD}$} \\
$\mathrm{S}$ & 0.783570308 & 0.701481955 & 0.116774755 \\
$\mathrm{~S}$ & -0.814500536 & -0.689246078 & 0.084280982 \\
$\mathrm{O}$ & -3.958052095 & 0.674623339 & 1.112084129 \\
$\mathrm{O}$ & 4.010807804 & -0.704996897 & 1.092053793 \\
$\mathrm{O}$ & -4.541201655 & -0.507794153 & -0.708874310 \\
$\mathrm{O}$ & 4.603160238 & 0.403810561 & -0.772842486 \\
$\mathrm{~N}$ & -2.156640761 & 2.148103239 & -0.226440787 \\
$\mathrm{~N}$ & 2.109673496 & -2.126123228 & -0.184098139 \\
$\mathrm{C}$ & -3.827667311 & 0.388088324 & -0.052532452 \\
$\mathrm{C}$ & 3.856905148 & -0.431502968 & -0.072830256 \\
$\mathrm{C}$ & -2.752075305 & 1.009707991 & -0.940886591 \\
$\mathrm{C}$ & 2.722775968 & -1.008085310 & -0.914015941 \\
$\mathrm{C}$ & -1.744712313 & -0.055582710 & -1.359189212 \\
$\mathrm{C}$ & 1.748132720 & 0.094357409 & -1.313158764 \\
$\mathrm{H}$ & -2.309337865 & 1.995162989 & 0.780743938 \\
$\mathrm{H}$ & 2.230526754 & -1.949121787 & 0.823752436 \\
$\mathrm{H}$ & -2.649621109 & 3.018846693 & -0.441842454 \\
$\mathrm{H}$ & 2.639126824 & -2.985839914 & -0.357527287 \\
$\mathrm{H}$ & -3.239873590 & 1.362787074 & -1.865095684 \\
$\mathrm{H}$ & 3.157363029 & -1.395064030 & -1.849308152 \\
$\mathrm{H}$ & -5.198023158 & -0.936964137 & -0.127983478 \\
$\mathrm{H}$ & 5.331857363 & 0.773337676 & -0.237114907 \\
$\mathrm{H}$ & -1.044927384 & 0.339412117 & -2.109229658 \\
$\mathrm{H}$ & 1.063562965 & -0.228250039 & -2.111358595 \\
$\mathrm{H}$ & 2.289649355 & 0.982991826 & -1.670318181 \\
$\mathrm{H}$ & -2.256063216 & -0.935030081 & -1.777670576 \\
$\mathrm{Pd}$ & -0.169012564 & 2.716303326 & -0.502409375 \\
$\mathrm{Pd}$ & 0.143449167 & -2.700857337 & -0.516822738 \\
$\mathrm{Cl}$ & 2.038170735 & 3.368953963 & -0.709582624 \\
$\mathrm{Cl}$ & -2.059512293 & -3.337980695 & -0.794888347 \\
$\mathrm{Cl}$ & -1.000870688 & 4.785883948 & -1.071105469 \\
$\mathrm{Cl}$ & 0.993359968 & -4.771413068 & -1.038960791 \\
& & & \\
& & $\mathrm{Cl} \cdot \mathrm{H}_{2} \mathrm{O}$ & \\
$\mathrm{O}$ & 0.061601224 & 0.130537347 & 1.567793193 \\
$\mathrm{H}$ & -0.586265351 & -0.052341121 & 0.838791208 \\
$\mathrm{H}$ & 0.818934256 & 0.418913455 & 1.054840783 \\
$\mathrm{O}$ & -1.523652359 & -0.271742781 & -1.041503454 \\
& 0.025656571 & 0.114842529 & 1.521119836
\end{tabular}




\begin{tabular}{|c|c|c|c|}
\hline $\mathrm{H}$ & -0.516772907 & -0.014106742 & 0.703279689 \\
\hline & 0.877019131 & 0.431806527 & 1.205655788 \\
\hline & -1.664752877 & -0.326048942 & -0.975955155 \\
\hline \multicolumn{4}{|c|}{$\mathrm{Cys} \cdot 6 \mathrm{H}_{2} \mathrm{O}$} \\
\hline z & -3.947873434 & -0.660527117 & 0.202418152 \\
\hline & 3.947873434 & 0.660527117 & \\
\hline & -3.240424928 & 0.397807529 & -0.64438 \\
\hline & 3.240424928 & -0.397807529 & -0.64 \\
\hline & -1.951371025 & -0.092039003 & \\
\hline & 1.951371025 & & \\
\hline & -2.404050813 & 1.350187668 & \\
\hline & 2.404050813 & -1.35018 & \\
\hline & -2.55 & & \\
\hline I & 2.590918001 & -2.3 & -0.3 \\
\hline H & -3.933327385 & 1.9 & \\
\hline & 3.93 & -1.9 & \\
\hline H & -3.9 & 0.6 & \\
\hline I & 2733294 & -0.68 & -1. \\
\hline $\mathrm{H}$ & 3773276 & 0.92 & -1.9 \\
\hline & $-2.1 \xi$ & -0.9 & \\
\hline I & -1.4 & & \\
\hline & 1.48 & -0.7 & -1.8 \\
\hline & 0853 & 2.4 & \\
\hline $\mathrm{H}$ & -4.5 & -2.4 & \\
\hline $\mathrm{N}$ & -3.0 & & \\
\hline $\mathrm{N}$ & 3.02 & -1.5 & 0.2 \\
\hline $\mathrm{O}$ & -4.3 & -0.4 & \\
\hline $\mathrm{O}$ & 4.3 & & \\
\hline $\mathrm{O}$ & & & -0. \\
\hline & -4.0 & -1.8 & -0. \\
\hline $\mathrm{S}$ & 0.7 & & \\
\hline $\mathrm{S}$ & -0.7 & -0.6 & -0. \\
\hline $\mathrm{O}$ & -5.4 & & \\
\hline $\mathrm{O}$ & 5.45 & -2.26 & \\
\hline $\mathrm{H}$ & -5.9 & 3.0 & \\
\hline $\mathrm{H}$ & 5.940 & -3.05 & 1.3 \\
\hline $\mathrm{H}$ & -5.781927953 & & 1.6 \\
\hline $\mathrm{H}$ & 7953 & -1.5 & \\
\hline $\mathrm{O}$ & 5.2 & 3.5 & \\
\hline $\mathrm{O}$ & -5.24 & -3.548634707 & 1.0 \\
\hline $\mathrm{H}$ & 5.852574820 & 4.215850733 & 0.6 \\
\hline & -5.8 & -4.2 & \\
\hline $\mathrm{H}$ & & & \\
\hline $\mathrm{H}$ & -5.597141653 & -3.254522611 & 1.86392805 \\
\hline $\mathrm{O}$ & -1.8673 & 3.45 & -1.52155510 \\
\hline $\mathrm{O}$ & & -3.450 & -1.5 \\
\hline $\mathrm{H}$ & -2.372722284 & 4.035695722 & -2.09517482 \\
\hline $\mathrm{H}$ & 2.372722284 & -4.035695722 & -2.09517482 \\
\hline & -1.034721596 & 3.902806951 & -1.35529478 \\
\hline & 1.034721596 & -3.902806951 & -1.35529478 \\
\hline
\end{tabular}




\begin{tabular}{|c|c|c|c|}
\hline \multicolumn{4}{|c|}{ Cys $6 \mathrm{H}_{2} \mathrm{O}$ SMD } \\
\hline $\mathrm{C}$ & 3.739546598 & 0.728450604 & 0.254954371 \\
\hline $\mathrm{C}$ & -4.030834141 & -0.653157512 & 0.247458911 \\
\hline $\mathrm{C}$ & 3.127402922 & -0.322240207 & -0.661229705 \\
\hline $\mathrm{C}$ & -3.179312816 & 0.258280159 & \\
\hline $\mathrm{C}$ & 1.732397444 & 0.064570027 & -1.127169915 \\
\hline$C$ & -1.956480256 & -0.441896767 & \\
\hline $\mathrm{H}$ & 2.577086066 & -1.586029576 & \\
\hline $\mathrm{H}$ & -2.317601953 & 1.191555851 & \\
\hline $\mathrm{H}$ & 2.773031295 & -2.376565986 & \\
\hline $\mathrm{H}$ & -2.245288937 & 2.114616526 & \\
\hline $\mathrm{H}$ & 4.116 & -1.857252541 & \\
\hline $\mathrm{H}$ & 1678593 & 605218 & \\
\hline $\mathrm{H}$ & 3.77 & -0.404980008 & \\
\hline $\mathrm{H}$ & -3.79 & 48280 & \\
\hline $\mathrm{H}$ & -2.28 & 61312 & \\
\hline $\mathrm{H}$ & 1.7 & 0.986349072 & \\
\hline $\mathrm{H}$ & 1.3 & -0.710 & \\
\hline $\mathrm{H}$ & -1.3 & 20444 & \\
\hline $\mathrm{H}$ & -4.85 & 64387 & \\
\hline $\mathrm{H}$ & & 2.606 & \\
\hline $\mathrm{N}$ & 3.1 & -1.62 & \\
\hline $\mathrm{N}$ & -2.8 & & \\
\hline $\mathrm{O}$ & 4.1 & 0.462 & \\
\hline $\mathrm{O}$ & -4.4 & 543941 & \\
\hline $\mathrm{O}$ & -4.2 & -1.8 & \\
\hline $\mathrm{O}$ & & & \\
\hline S & -0.8 & 8264 & \\
\hline S & 0.5 & & \\
\hline $\mathrm{O}$ & 5.7 & -1.98 & \\
\hline $\mathrm{O}$ & $-5.2 ?$ & 2198 & \\
\hline $\mathrm{H}$ & & -2.81 & \\
\hline $\mathrm{H}$ & -5.2 & & \\
\hline $\mathrm{H}$ & 5.8 & -1.30 & \\
\hline $\mathrm{H}$ & -5.5 & 5918 & \\
\hline $\mathrm{O}$ & -5.71 & 96064 & \\
\hline $\mathrm{O}$ & & & \\
\hline $\mathrm{H}$ & -6.39 & -3.840 & \\
\hline $\mathrm{H}$ & 710641 & 4.312360556 & 0.7 \\
\hline $\mathrm{H}$ & -6.15 & -2.95 & \\
\hline $\mathrm{H}$ & 5.1 & 5197 & \\
\hline $\mathrm{O}$ & 2.2 & 56285 & \\
\hline $\mathrm{O}$ & -1.3 & 63050 & \\
\hline $\mathrm{H}$ & & & \\
\hline $\mathrm{H}$ & -1.8 & 3.742065888 & \\
\hline $\mathrm{H}$ & $1.3716^{?}$ & -3.907076381 & -1.6 \\
\hline $\mathrm{H}$ & -0.588086343 & 3.580023316 & -1.04222817 \\
\hline & & & \\
\hline$P 0$ & & & \\
\hline $\mathrm{Cl}$ & & 0.000000000 & \\
\hline $\mathrm{Cl}$ & 0.000000000 & -2.339894041 & 0.00000000 \\
\hline
\end{tabular}




$\begin{array}{lrrr}\mathrm{Cl} & 0.000000000 & 2.339894041 & 0.000000000 \\ \mathrm{Cl} & -2.339894041 & 0.000000000 & 0.000000000 \\ \mathrm{O} & 2.894328407 & 2.894328407 & 1.436007303 \\ \mathrm{O} & -2.894328407 & 2.894328407 & -1.436007303 \\ \mathrm{O} & 2.894328407 & -2.894328407 & -1.436007303 \\ \mathrm{O} & -2.894328407 & -2.894328407 & 1.436007303 \\ \mathrm{H} & 3.028286517 & 1.985753066 & 1.126332860 \\ \mathrm{H} & -3.028286517 & 1.985753066 & -1.126332860 \\ \mathrm{H} & 3.028286517 & -1.985753066 & -1.126332860 \\ \mathrm{H} & -1.985753066 & -3.028286517 & 1.126332860 \\ \mathrm{H} & 1.985753066 & 3.028286517 & 1.126332860 \\ \mathrm{H} & -1.985753066 & 3.028286517 & -1.126332860 \\ \mathrm{H} & 1.985753066 & -3.028286517 & -1.126332860 \\ \mathrm{H} & -3.028286517 & -1.985753066 & 1.126332860\end{array}$

$\mathrm{PdCl}_{4} \cdot 4 \mathrm{H}_{2} \mathrm{O}$ SMD

$\begin{array}{lrrr}\mathrm{Pd} & -0.002623389 & 0.011996201 & -0.003048942 \\ \mathrm{Cl} & -2.334020994 & 0.005097822 & -0.015799701 \\ \mathrm{Cl} & -0.001807626 & 2.338798363 & 0.000813899 \\ \mathrm{Cl} & -0.004082865 & -2.311182557 & -0.008045572 \\ \mathrm{Cl} & 2.328144871 & 0.004122897 & 0.012651449 \\ \mathrm{O} & -1.970205688 & -1.988157990 & 2.526001289 \\ \mathrm{O} & 1.991413187 & -2.003009564 & -2.523535870 \\ \mathrm{O} & -1.994456219 & 1.987832350 & -2.518089684 \\ \mathrm{O} & 1.984157182 & 1.976600424 & 2.523606768 \\ \mathrm{H} & -2.351355922 & -1.289486620 & 1.973808141 \\ \mathrm{H} & 2.363931742 & -1.302088819 & -1.968175490 \\ \mathrm{H} & -2.335762585 & 1.275055329 & -1.955727805 \\ \mathrm{H} & 1.281253418 & 2.339382886 & 1.963184962 \\ \mathrm{H} & -1.267436187 & -2.323597916 & 1.948373656 \\ \mathrm{H} & 1.277131746 & -2.333820375 & -1.957677767 \\ \mathrm{H} & -1.289640129 & 2.347834147 & -1.958342085 \\ \mathrm{H} & 2.325359457 & 1.264623421 & 1.960002752\end{array}$

\begin{tabular}{lrrr} 
& \multicolumn{4}{c}{$\left[\mathrm{PdCl}_{3}\left(\mathrm{~S}-\mathrm{H}_{4} \mathrm{CysS}\right)\right]^{+} \cdot 9 \mathrm{H}_{2} \mathrm{O}$} \\
$\mathrm{C}$ & 3.724327901 & -0.592753996 & -1.393393411 \\
$\mathrm{C}$ & -4.073306185 & 0.753228048 & -1.924244143 \\
$\mathrm{C}$ & 2.677821493 & -1.712991125 & -1.494557430 \\
$\mathrm{C}$ & -2.978840673 & 1.799265149 & -2.181069439 \\
$\mathrm{C}$ & 1.372364716 & -1.331970817 & -2.163429628 \\
$\mathrm{C}$ & -1.872779680 & 1.269261380 & -3.084130249 \\
$\mathrm{H}$ & 2.278860888 & -1.642394321 & 0.529027233 \\
$\mathrm{H}$ & -2.173775196 & 1.423350929 & -0.320386474 \\
$\mathrm{H}$ & 1.676804009 & -3.034163256 & -0.238106410 \\
$\mathrm{H}$ & -1.628795466 & 2.869423846 & -0.998021496 \\
$\mathrm{H}$ & 3.383403003 & -2.856377376 & 0.107363025 \\
$\mathrm{H}$ & -3.218345069 & 2.701078377 & -0.248023254 \\
$\mathrm{H}$ & 3.130548826 & -2.474127604 & -2.154771536 \\
$\mathrm{H}$ & -3.443497515 & 2.667991189 & -2.679320701 \\
$\mathrm{H}$ & 1.574571945 & -1.014112152 & -3.194280827 \\
$\mathrm{H}$ & -2.266666768 & 1.125005481 & -4.099764029 \\
$\mathrm{H}$ & 0.711313958 & -2.210576436 & -2.159593466
\end{tabular}




\begin{tabular}{|c|c|c|c|}
\hline $\mathrm{H}$ & -1.023845399 & 968812116 & -3.123300742 \\
\hline & 4.028607765 & 1.234795435 & -1.963780299 \\
\hline & -4.619484553 & -0.953652543 & -2.543379962 \\
\hline & 2.482986698 & -2.365936617 & -0.183660546 \\
\hline & -2.458969593 & 2.245498584 & -0.881877266 \\
\hline & 4.714502802 & -0.708841693 & -0.702425503 \\
\hline & -4.699356698 & 0.729111905 & -0.889265689 \\
\hline $\mathrm{O}$ & 3.431744327 & 0.408825165 & -2.159399860 \\
\hline & -4.191802073 & -0.095302787 & -2.90822070 \\
\hline S & 0.412918404 & 0.073268022 & -1.455 \\
\hline$S$ & -1.285631624 & -0.382509323 & -2.59 \\
\hline O & 4.937775199 & -3.266386245 & \\
\hline $\mathrm{O}$ & -4.214882364 & 3.00 & \\
\hline & 5.420043829 & -3.57 & \\
\hline $\mathrm{H}$ & -3.5 & 2.7 & \\
\hline & 5.257 & -2.36 & -0.0 \\
\hline H & -4.7805780 & 2.23 & \\
\hline $\mathrm{O}$ & 4.5 & 2.51 & \\
\hline $\mathrm{O}$ & -4.85 & $-2.1 \xi$ & \\
\hline & $-4.0^{\prime}$ & -2.65 & \\
\hline$H$ & 3.8 & 2.9 & \\
\hline $\mathrm{H}$ & 5.28 & 2.35 & \\
\hline & -5.21 & -1.78 & -0.8 \\
\hline & 0.02 & -0.1 & \\
\hline $\mathrm{Cl}$ & -0.24 & -0.0 & \\
\hline $\mathrm{Cl}$ & 2.2 & 0.4 & \\
\hline $\mathrm{Cl}$ & -2.2 & -0.5 & 0.6 \\
\hline $\mathrm{O}$ & -2.43 & -3.25 & \\
\hline $\mathrm{O}$ & 2.2 & 3.2 & \\
\hline $\mathrm{H}$ & -2.3 & -2.4 & \\
\hline $\mathrm{H}$ & & 2.33 & \\
\hline $\mathrm{H}$ & -2.7 & -3.8 & \\
\hline $\mathrm{H}$ & 2.2 & 3.8 & \\
\hline $\mathrm{O}$ & & -3.89 & \\
\hline $\mathrm{O}$ & -0.13 & & \\
\hline I & -0.7 & -3.6 & \\
\hline $\mathrm{H}$ & & 3.42 & -1.1 \\
\hline $\mathrm{H}$ & 0.29 & -4.81 & -1.0 \\
\hline & -0.08 & 4.48 & \\
\hline $\mathrm{O}$ & -1.9 & 2.4 & \\
\hline $\mathrm{H}$ & -1.6 & 1.57 & \\
\hline $\mathrm{H}$ & -1.547417963 & 3.086896750 & 2.76046873 \\
\hline \multicolumn{4}{|c|}{$\left[\mathrm{PdCl}_{3}\left(\mathrm{~S}-\mathrm{H}_{4} \mathrm{CysS}\right)\right]^{+} \cdot 9 \mathrm{H}_{2} \mathrm{O} \mathrm{SMD}$} \\
\hline $\mathrm{C}$ & 3.722816228 & -0.590774524 & \\
\hline $\mathrm{C}$ & -4.0863 & 0.80 & -1.8 \\
\hline $\mathrm{C}$ & & -1.705755808 & \\
\hline $\mathrm{C}$ & -2.967931619 & 1.802561903 & -2.12929074 \\
\hline $\mathrm{C}$ & 1.367798415 & -1.321986011 & -2.11137694 \\
\hline $\mathrm{C}$ & -1.889339277 & 1.215092195 & \\
\hline 11 & 2.307959561 & -1.744233750 & 0.55810595 \\
\hline $\mathrm{H}$ & -2.138935766 & 1.475344115 & -0.25942658 \\
\hline
\end{tabular}




\begin{tabular}{|c|c|c|c|}
\hline $\mathrm{H}$ & 1.683608033 & -3.074620627 & -0.277659038 \\
\hline $\mathrm{H}$ & -1.540885780 & 2.868214821 & -1.024360971 \\
\hline $\mathrm{H}$ & 3.388575458 & -2.940695393 & 0.065739543 \\
\hline $\mathrm{H}$ & -3.110512470 & 2.787235535 & -0.267991229 \\
\hline $\mathrm{H}$ & 3.128739775 & -2.427486887 & -2.181071272 \\
\hline $\mathrm{H}$ & -3.412452537 & 2.667906609 & -2.652494365 \\
\hline $\mathrm{H}$ & 1.547719796 & -0.985769731 & -3.140852153 \\
\hline $\mathrm{H}$ & -2.302445299 & 1.046100653 & -4.032837352 \\
\hline $\mathrm{H}$ & 0.720493944 & -2.210124337 & -2.118303916 \\
\hline $\mathrm{H}$ & -1.036022697 & 1.903805815 & -3.117546792 \\
\hline $\mathrm{H}$ & 4.020877757 & 1.271973195 & -1.877378957 \\
\hline $\mathrm{H}$ & -4.667205656 & -0.953244359 & -2.414973916 \\
\hline $\mathrm{N}$ & 2.500675011 & -2.422878452 & -0.194869635 \\
\hline $\mathrm{N}$ & -2.402359554 & 2.278125413 & -0.856832840 \\
\hline $\mathrm{O}$ & 4.724125550 & -0.731548277 & -0.658920179 \\
\hline $\mathrm{O}$ & -4.710020015 & 0.846763296 & -0.776282910 \\
\hline $\mathrm{O}$ & 3.428688400 & 0.438856938 & -2.064980565 \\
\hline $\mathrm{O}$ & -4.247958927 & -0.077039283 & -2.765205904 \\
\hline $\mathrm{S}$ & 0.385192348 & 0.050459790 & -1.372448038 \\
\hline S & -1.314510549 & -0.423493343 & -2.496774282 \\
\hline $\mathrm{O}$ & 4.990559878 & -3.336409928 & 0.229557856 \\
\hline $\mathrm{O}$ & -4.179719856 & 3.031294245 & 1.062922529 \\
\hline $\mathrm{H}$ & 5.276074321 & -3.454352706 & 1.146994511 \\
\hline $\mathrm{H}$ & -3.411445262 & 2.787561543 & 1.627936952 \\
\hline $\mathrm{H}$ & 5.242131960 & -2.421246434 & -0.002912644 \\
\hline $\mathrm{H}$ & -4.582009190 & 2.185186161 & 0.806990576 \\
\hline $\mathrm{O}$ & 4.506872684 & 2.624393433 & -1.552748683 \\
\hline $\mathrm{O}$ & -4.887269223 & -2.357403982 & -1.945391959 \\
\hline $\mathrm{H}$ & -4.050004263 & -2.724699823 & -1.580548417 \\
\hline $\mathrm{H}$ & 3.726442239 & 2.994174477 & -1.074548809 \\
\hline $\mathrm{H}$ & 5.229205324 & 2.619376242 & -0.908296851 \\
\hline $\mathrm{H}$ & -5.537215860 & -2.426795142 & -1.231249797 \\
\hline $\mathrm{Pd}$ & 0.012209030 & -0.145076753 & 0.934285006 \\
\hline $\mathrm{Cl}$ & -0.255488034 & -0.086861814 & 3.236469494 \\
\hline $\mathrm{Cl}$ & 2.264586766 & 0.409271765 & 1.193459669 \\
\hline $\mathrm{Cl}$ & -2.260766452 & -0.555156056 & 0.770646938 \\
\hline $\mathrm{O}$ & -2.540202306 & -3.225871970 & -0.694595493 \\
\hline $\mathrm{O}$ & 2.229436524 & 3.203590416 & -0.198305361 \\
\hline $\mathrm{H}$ & -2.458124855 & -2.378860263 & -0.201687421 \\
\hline $\mathrm{H}$ & 2.187595594 & 2.353051300 & 0.287892710 \\
\hline $\mathrm{H}$ & -2.851223621 & -3.870828206 & -0.040067489 \\
\hline $\mathrm{H}$ & 2.257578968 & 3.889648812 & 0.486795377 \\
\hline $\mathrm{O}$ & 0.189409009 & -3.885994666 & -0.643915722 \\
\hline $\mathrm{O}$ & -0.125551463 & 3.524916453 & -1.591983868 \\
\hline $\mathrm{H}$ & -0.753379503 & -3.625485982 & -0.719273639 \\
\hline $\mathrm{H}$ & 0.721727172 & 3.393695879 & -1.110431268 \\
\hline $\mathrm{H}$ & 0.260362621 & -4.702998190 & -1.156693996 \\
\hline $\mathrm{H}$ & -0.164592480 & 4.470856235 & -1.788809750 \\
\hline $\mathrm{O}$ & -1.713942603 & 2.493005155 & 2.004019413 \\
\hline $\mathrm{H}$ & -1.406245329 & 1.736906540 & 2.538542394 \\
\hline $\mathrm{H}$ & -1.206525850 & 3.250300325 & 2.326752996 \\
\hline
\end{tabular}




\begin{tabular}{|c|c|c|c|}
\hline \multicolumn{4}{|c|}{$\left[\mathrm{PdCl}_{3}\left(\mathrm{~N}-\mathrm{H}_{4} \mathrm{CysS}\right)\right] \cdot 9 \mathrm{H}_{2} \mathrm{O}$} \\
\hline $\mathrm{C}$ & -3.506955096 & -1.000870035 & 0.039961780 \\
\hline $\mathrm{C}$ & 3.999947819 & 0.733868729 & 0.556257164 \\
\hline $\mathrm{C}$ & 3.128928546 & -0.118353843 & -0.360409926 \\
\hline $\mathrm{C}$ & -2.789375033 & -0.072939688 & -0.962630426 \\
\hline $\mathrm{C}$ & 2.085800038 & 0.744126622 & -1.066178335 \\
\hline $\mathrm{C}$ & -1.301668831 & -0.363645219 & -1.080878402 \\
\hline $\mathrm{H}$ & 2.089611878 & -0.879147457 & 1.207684388 \\
\hline $\mathrm{H}$ & -2.551075579 & 1.473116222 & 0.339167116 \\
\hline $\mathrm{H}$ & -2.534584600 & 1.979773058 & -1.243759162 \\
\hline $\mathrm{H}$ & 3.397338350 & -1.809876533 & 0.763595061 \\
\hline $\mathrm{H}$ & -4.047366267 & 1.574095862 & -0.468826816 \\
\hline $\mathrm{H}$ & 3.783510755 & -0.495996605 & -1.164823145 \\
\hline $\mathrm{H}$ & -3.266218408 & -0.226247843 & -1.945419435 \\
\hline $\mathrm{H}$ & -1.143540216 & -1.431250663 & -1.296356657 \\
\hline $\mathrm{H}$ & 2.594410216 & 1.584702640 & -1.563013212 \\
\hline $\mathrm{H}$ & 1.584117925 & 0.135609128 & -1.833301528 \\
\hline $\mathrm{H}$ & -0.846102098 & 0.222863474 & -1.890137708 \\
\hline $\mathrm{H}$ & -3.331156565 & -2.863011378 & 0.884354008 \\
\hline $\mathrm{H}$ & 5.198542077 & 2.185158749 & 0.581657167 \\
\hline $\mathrm{N}$ & 2.596098892 & -1.241807845 & 0.383928067 \\
\hline $\mathrm{N}$ & -3.016372329 & 1.336188007 & -0.569987961 \\
\hline $\mathrm{O}$ & 4.082063558 & 0.549965936 & 1.757031388 \\
\hline $\mathrm{O}$ & -4.494719382 & -0.611638145 & 0.650271090 \\
\hline $\mathrm{O}$ & -2.961251461 & -2.148600278 & 0.106561008 \\
\hline $\mathrm{O}$ & 4.636432225 & 1.684097555 & -0.086953625 \\
\hline S & -0.441021628 & -0.002051241 & 0.498458857 \\
\hline S & 0.841204303 & 1.552120698 & -0.009993376 \\
\hline $\mathrm{O}$ & 4.462239375 & -3.078428510 & 1.147078846 \\
\hline $\mathrm{O}$ & -5.669806776 & 1.578071460 & -0.260266134 \\
\hline $\mathrm{H}$ & 4.521519692 & -3.421333913 & 0.219003949 \\
\hline $\mathrm{H}$ & -6.091101817 & 2.131586906 & 0.406483087 \\
\hline $\mathrm{H}$ & 3.776941652 & -3.626111519 & 1.555163650 \\
\hline $\mathrm{H}$ & -5.536016812 & 0.702218692 & 0.169648581 \\
\hline $\mathrm{O}$ & -3.535997172 & -3.756892672 & 1.795715023 \\
\hline $\mathrm{O}$ & 5.891030986 & 2.582595094 & 2.002067308 \\
\hline $\mathrm{H}$ & -2.658678647 & -4.304625394 & 1.817416694 \\
\hline $\mathrm{H}$ & 6.792312380 & 2.252609544 & 2.099675629 \\
\hline $\mathrm{H}$ & -4.229998975 & -4.377067352 & 1.546872899 \\
\hline $\mathrm{H}$ & 5.331537121 & 1.890662676 & 2.406131411 \\
\hline $\mathrm{O}$ & -1.273607790 & 2.673848068 & -2.147544113 \\
\hline $\mathrm{H}$ & -1.153867826 & 3.499485187 & -2.627843563 \\
\hline $\mathrm{H}$ & -0.466733700 & 2.542676214 & -1.606934055 \\
\hline $\mathrm{Pd}$ & 1.341813747 & -2.701324915 & -0.346794469 \\
\hline $\mathrm{Cl}$ & -0.208420196 & -4.301670784 & -1.054097338 \\
\hline $\mathrm{Cl}$ & 1.770872393 & -2.143359772 & -2.580636209 \\
\hline $\mathrm{Cl}$ & 1.200813811 & -3.368413926 & 1.920648038 \\
\hline $\mathrm{O}$ & -1.395193351 & -5.069839273 & 1.686497707 \\
\hline $\mathrm{H}$ & -0.646307202 & -4.627027998 & 2.127966331 \\
\hline $\mathrm{H}$ & -1.102574223 & -5.002672591 & 0.750620792 \\
\hline $\mathrm{O}$ & 2.466268042 & -5.362649080 & -2.372169693 \\
\hline $\mathrm{H}$ & 2.142278882 & -4.576878689 & -2.840846344 \\
\hline
\end{tabular}




$\begin{array}{lllr}\mathrm{H} & 1.738743532 & -5.498991676 & -1.744319320 \\ \mathrm{O} & 1.460327740 & -0.323784064 & 2.885167274 \\ \mathrm{H} & 2.405914999 & -0.171426537 & 3.019215894 \\ \mathrm{H} & 1.347382489 & -1.286136449 & 2.972509044 \\ \mathrm{O} & 4.443996145 & -3.761480491 & -1.448352979 \\ \mathrm{H} & 3.832649973 & -4.502355256 & -1.673055520 \\ \mathrm{H} & 4.042682723 & -3.036913157 & -1.949660837\end{array}$

$\left[\mathrm{PdCl}_{3}\left(\mathrm{~N}-\mathrm{H}_{4} \mathrm{CysS}\right)\right] \cdot 9 \mathrm{H}_{2} \mathrm{O} \quad \mathrm{SMD}$

$\begin{array}{lrrr}\mathrm{C} & -3.510641370 & -0.986541869 & 0.050197927 \\ \mathrm{C} & 4.059015729 & 0.680318348 & 0.509863802 \\ \mathrm{C} & 3.162473636 & -0.156311698 & -0.398531641 \\ \mathrm{C} & -2.799463954 & -0.022094379 & -0.902691965 \\ \mathrm{C} & 2.106457544 & 0.712171168 & -1.072531444 \\ \mathrm{C} & -1.309633967 & -0.287848836 & -1.040157566 \\ \mathrm{H} & 2.108791250 & -0.903409956 & 1.174882809 \\ \mathrm{H} & -2.800587320 & 1.525204505 & 0.479662720 \\ \mathrm{H} & -2.531085301 & 2.036866766 & -1.097598246 \\ \mathrm{H} & 3.414812433 & -1.819178832 & 0.771205427 \\ \mathrm{H} & -4.116912909 & 1.580702567 & -0.551552940 \\ \mathrm{H} & 3.795160263 & -0.553361854 & -1.210379370 \\ \mathrm{H} & -3.261201682 & -0.177438944 & -1.894161033 \\ \mathrm{H} & -1.152425160 & -1.336255315 & -1.328859361 \\ \mathrm{H} & 2.603383731 & 1.544846337 & -1.594303391 \\ \mathrm{H} & 1.571991895 & 0.119880276 & -1.828163826 \\ \mathrm{H} & -0.879855179 & 0.349551458 & -1.825507749 \\ \mathrm{H} & -3.371967739 & -2.810615195 & 0.790898171 \\ \mathrm{H} & 5.192203225 & 2.196743772 & 0.566894741 \\ \mathrm{~N} & 2.625586727 & -1.276022705 & 0.364930867 \\ \mathrm{~N} & -3.082240252 & 1.366307899 & -0.495026205 \\ \mathrm{O} & 4.194531704 & 0.444957896 & 1.699456983 \\ \mathrm{O} & -4.531813153 & -0.663319977 & 0.635958805 \\ \mathrm{O} & -2.924053674 & -2.133161273 & 0.122013597 \\ \mathrm{O} & 4.647334562 & 1.665222950 & -0.114264755 \\ \mathrm{~S} & -0.396226324 & -0.010842891 & 0.521182894 \\ \mathrm{~S} & 0.906102019 & 1.526421942 & 0.028259321 \\ \mathrm{O} & 4.392905732 & -3.284824043 & 1.257512866 \\ \mathrm{O} & -5.791354388 & 1.551778498 & -0.467096519 \\ \mathrm{H} & 4.459788535 & -3.603560102 & 0.322940673 \\ \mathrm{H} & -6.149850366 & 2.191692100 & 0.164274440 \\ \mathrm{H} & 3.565130925 & -3.674578878 & 1.581445907 \\ \mathrm{H} & -5.721094446 & 0.712873746 & 0.025352010 \\ \mathrm{O} & -3.742869391 & -3.837393305 & 1.721021581 \\ \mathrm{O} & 5.846139943 & 2.829551445 & 1.815206767 \\ \mathrm{H} & -2.885231603 & -4.354693918 & 1.775928796 \\ \mathrm{H} & 6.809403627 & 2.740626128 & 1.868866958 \\ \mathrm{H} & -4.381717688 & -4.446234958 & 1.323615183 \\ \mathrm{H} & 5.496813964 & 2.188401249 & 2.455560652 \\ \mathrm{O} & -1.390679216 & 2.799869281 & -2.055696200 \\ \mathrm{H} & -1.525093593 & 3.745834093 & -2.209928990 \\ \mathrm{H} & -0.534802465 & 2.730538065 & -1.597647219 \\ \mathrm{Pd} & 1.378786572 & -2.737658115 & -0.362435654 \\ & & & \end{array}$




$\begin{array}{lrrr}\mathrm{Cl} & -0.148322559 & -4.364679977 & -1.074465211 \\ \mathrm{Cl} & 1.925649377 & -2.265687011 & -2.587546090 \\ \mathrm{Cl} & 1.201959049 & -3.403917524 & 1.904236592 \\ \mathrm{O} & -1.447817325 & -5.088032200 & 1.660695999 \\ \mathrm{H} & -0.750217542 & -4.609997988 & 2.143167300 \\ \mathrm{H} & -1.146828691 & -4.951872351 & 0.737570631 \\ \mathrm{O} & 2.501546257 & -5.430705611 & -2.365349200 \\ \mathrm{H} & 2.249831434 & -4.602584766 & -2.812190088 \\ \mathrm{H} & 1.759629264 & -5.523769770 & -1.742049675 \\ \mathrm{O} & 1.851046094 & -0.451384437 & 3.087349615 \\ \mathrm{H} & 2.778112128 & -0.233751402 & 2.893819025 \\ \mathrm{H} & 1.813699942 & -1.421219023 & 3.072844119 \\ \mathrm{O} & 4.537643559 & -3.966895287 & -1.343592171 \\ \mathrm{H} & 3.841550584 & -4.618811435 & -1.599013433 \\ \mathrm{H} & 4.274125840 & -3.187084929 & -1.854458271\end{array}$

$\left[\mathrm{PdCl}_{3}\left(\mathrm{~S}-\mathrm{H}_{2} \mathrm{Cys}\right)\right]^{-}$

$\begin{array}{lrrr}\mathrm{S} & 7.836844852 & 0.487918365 & 3.255642304 \\ \mathrm{C} & 8.715447365 & -0.464771029 & 1.981093542 \\ \mathrm{C} & 8.638091225 & -1.983933276 & 2.263056133 \\ \mathrm{~N} & 7.318362207 & -2.299514028 & 2.812594455 \\ \mathrm{H} & 9.774991326 & -0.172831182 & 1.948888305 \\ \mathrm{H} & 8.259419485 & -0.284415571 & 0.998040344 \\ \mathrm{H} & 8.781100044 & -2.538063392 & 1.317957873 \\ \mathrm{H} & 6.586441067 & -2.132185152 & 2.042450814 \\ \mathrm{H} & 7.286450258 & -3.203354263 & 3.286818741 \\ \mathrm{Pd} & 5.539881865 & 0.490460052 & 2.614445162 \\ \mathrm{Cl} & 5.795000191 & -0.985872959 & 0.703733471 \\ \mathrm{Cl} & 3.254768165 & 0.575674507 & 2.200306297 \\ \mathrm{H} & 7.142391037 & -1.485006661 & 3.488729552 \\ \mathrm{Cl} & 5.317805143 & 1.767741761 & 4.524143875 \\ \mathrm{C} & 9.667485848 & -2.506566303 & 3.240517547 \\ \mathrm{O} & 9.416130445 & -3.265057948 & 4.144264561 \\ \mathrm{O} & 10.901799136 & -2.093092077 & 2.962745392 \\ \mathrm{H} & 11.480475164 & -2.485145896 & 3.634195870\end{array}$

\begin{tabular}{lrrr} 
& \multicolumn{3}{c}{$\left[\mathrm{PdCl}_{3}\left(\mathrm{~S}-\mathrm{H}_{2} \mathrm{Cys}\right)\right]^{-} \mathrm{SMD}$} \\
$\mathrm{S}$ & 7.838000647 & 0.406468280 & 3.205676860 \\
$\mathrm{C}$ & 8.658862878 & -0.563455579 & 1.901879781 \\
$\mathrm{C}$ & 8.697409990 & -2.058127109 & 2.234875158 \\
$\mathrm{~N}$ & 7.351458577 & -2.513335958 & 2.635919243 \\
$\mathrm{H}$ & 9.695423371 & -0.215425811 & 1.786783592 \\
$\mathrm{H}$ & 8.143518359 & -0.426872861 & 0.941517424 \\
$\mathrm{H}$ & 9.015784545 & -2.637760406 & 1.352325889 \\
$\mathrm{H}$ & 6.655397438 & -2.211250431 & 1.906518934 \\
$\mathrm{H}$ & 7.292477718 & -3.519303457 & 2.827735208 \\
$\mathrm{Pd}$ & 5.560434636 & 0.593139224 & 2.705964814 \\
$\mathrm{Cl}$ & 5.627280714 & -0.796458548 & 0.787077254 \\
$\mathrm{Cl}$ & 3.245951857 & 0.869530734 & 2.363857331 \\
$\mathrm{H}$ & 7.094607168 & -1.975204638 & 3.482531445 \\
$\mathrm{Cl}$ & 5.500282557 & 1.943188314 & 4.614763153 \\
$\mathrm{C}$ & 9.646143909 & -2.367157141 & 3.374153079
\end{tabular}


$\begin{array}{ccc}\mathrm{O} & 9.301341928 & -2.867602176 \\ \mathrm{O} & 10.889584108 & -2.020596387 \\ \mathrm{H} & 11.498924421 & -2.217791102\end{array}$

$\left[\mathrm{PdCl}_{3}\left(\mathrm{~S}-\mathrm{H}_{2} \mathrm{HCys}\right)\right]^{-}$

$\begin{array}{lll}\mathrm{S} & 0.000558608 & 1.317488962\end{array}$

O $\quad-3.072368908 \quad 3.584150423$

O $\quad-3.115399370$

$\mathrm{N} \quad-0.471777506$

C $\quad-2.495843874$

C $\quad-1.000745162$

C -0.621896995

C $\quad-0.933155366$

$\mathrm{H}-0.605917823$

$\mathrm{H} \quad 0.461949076$

$\mathrm{H} \quad-1.136164606$

$\mathrm{H} \quad-0.626182929$

$\mathrm{H} \quad-2.012008188$

$\mathrm{H} \quad-0.433699361$

$\mathrm{H} \quad-1.025389174$

$\mathrm{H} \quad 0.565446192$

$\mathrm{H} \quad-4.051865152$

$\mathrm{Pd} \quad 2.185292928$

$\mathrm{Cl} \quad 4.290203949$

$\mathrm{Cl} 2.292647762$

$\mathrm{Cl} \quad 1.969776527$
4.417296844

3.072209765

3.808538463

$-1.219897426$

1.320522615

$-0.327967359$

1.015433591

0.469411421

0.223473387

$-1.261411526$

$-1.940795260$

0.612251224

$-1.366032308$

$-1.779629094$

$-2.994336100$

$-1.897025581$

0.329515042

1.858344453

1.232526999

$-0.078787168$

$-1.169896316$

$-1.156591500$

1.207532344

$-3.452472671$

$\left[\mathrm{PdCl}_{3}\left(\mathrm{~S}-\mathrm{H}_{2} \mathrm{HCys}\right)\right]^{-} \mathrm{SMD}$

$\mathrm{S}$

$-0.080384701$

1.471650493

$-1.211168405$

O $\quad-3.040469835$

3.145902423

1.135918799

O $\quad-3.288088765$

4.746770376

$-0.417329593$

N $\quad-0.392736596$

3.285088923

1.138380375

C $\quad-2.576780914$

3.982492971

0.398497995

C $\quad-1.090825381$

4.281953189

0.300373539

C $\quad-0.591393580$

4.249108496

$-1.145888289$

C $\quad-0.881033023$

2.963096106

$-1.909293372$

H $\quad-0.930636039$

5.284963995

0.728833643

$\mathrm{H} \quad 0.494264154$

4.439250363

$-1.150487186$

H $\quad-1.065000109$

5.096150523

$-1.665755811$

$\mathrm{H} \quad-0.519855523$

3.078273003

$-2.941326370$

$\mathrm{H} \quad-1.966354782$

2.765141244

$-1.953136708$

$\mathrm{H} \quad-0.383810936$

2.361624725

0.588187034

$\mathrm{H} \quad-0.844661331$

3.157174526

2.049624869

$\mathrm{H} \quad 0.614971391$

$\mathrm{H} \quad-4.236378075$

3.454677045

1.266500535

$\mathrm{Pd} \quad 2.181349669$

4.524538085

$-0.358197943$

$\mathrm{Cl} \quad 4.500921933$

2.041872557

$-1.294870970$

Cl 2.319987697

2.499607493

$-1.397693723$

$\mathrm{Cl}$

1.764154947

1.044231976

$2.441830281-3.581231629$

$\left[\mathrm{PdCl}_{3}\left(\mathrm{~S}-\mathrm{H}_{2}\right.\right.$ Cyste $\left.)\right]$ 


$\begin{array}{lrrr}\mathrm{C} & 8.753279686 & -0.621580513 & 1.883364274 \\ \mathrm{C} & 8.623359339 & -2.126763664 & 2.134899571 \\ \mathrm{~N} & 7.246808183 & -2.396796145 & 2.586148506 \\ \mathrm{H} & 9.824245655 & -0.368367561 & 1.831940722 \\ \mathrm{H} & 8.281862225 & -0.370596132 & 0.921380176 \\ \mathrm{H} & 9.307420930 & -2.444054165 & 2.938096997 \\ \mathrm{H} & 8.826883722 & -2.722884056 & 1.230212374 \\ \mathrm{H} & 6.557599296 & -2.099944514 & 1.809956839 \\ \mathrm{H} & 7.074516845 & -3.335623246 & 2.941669362 \\ \mathrm{Pd} & 5.644336588 & 0.444627880 & 2.760119672 \\ \mathrm{Cl} & 5.734193840 & -0.790517982 & 0.672420337 \\ \mathrm{Cl} & 3.330785057 & 0.592088128 & 2.529406622 \\ \mathrm{H} & 7.073071806 & -1.642025928 & 3.318236279 \\ \mathrm{Cl} & 5.581426641 & 1.505825182 & 4.810503675\end{array}$

\begin{tabular}{lrrr} 
& \multicolumn{3}{c}{$\left[\mathrm{PdCl}_{3}\left(\mathrm{~S}-\mathrm{H}_{2} \mathrm{Cyste}\right)\right] \mathrm{SMD}$} \\
$\mathrm{S}$ & 7.869715854 & 0.317014169 & 3.243325501 \\
$\mathrm{C}$ & 8.682756541 & -0.621720880 & 1.905569471 \\
$\mathrm{C}$ & 8.682408094 & -2.122915108 & 2.151694100 \\
$\mathrm{~N}$ & 7.324971087 & -2.563448184 & 2.546281132 \\
$\mathrm{H}$ & 9.728436796 & -0.283860008 & 1.838077018 \\
$\mathrm{H}$ & 8.193297458 & -0.398197628 & 0.946779202 \\
$\mathrm{H}$ & 9.368463253 & -2.398119461 & 2.965730230 \\
$\mathrm{H}$ & 8.976264369 & -2.680367547 & 1.250304380 \\
$\mathrm{H}$ & 6.631436918 & -2.237057286 & 1.830767354 \\
$\mathrm{H}$ & 7.242977056 & -3.570662258 & 2.715500940 \\
$\mathrm{Pd}$ & 5.593935002 & 0.559241170 & 2.778965311 \\
$\mathrm{Cl}$ & 5.602753297 & -0.739755780 & 0.792069863 \\
$\mathrm{Cl}$ & 3.274468646 & 0.903694679 & 2.485967466 \\
$\mathrm{H}$ & 7.069822048 & -2.032652775 & 3.398647814 \\
$\mathrm{Cl}$ & 5.589553494 & 1.824522302 & 4.747210141
\end{tabular}

\begin{tabular}{lrrr} 
& \multicolumn{4}{c}{$\left[\mathrm{PdCl}_{3}\left(\mathrm{~S}_{-} \mathrm{H}_{2} \mathrm{MPA}\right)\right]^{-}$} \\
$\mathrm{S}$ & -3.416847621 & 1.214697220 & 0.852874322 \\
$\mathrm{C}$ & -1.904711359 & 2.190041106 & 1.119464629 \\
$\mathrm{C}$ & -1.424726266 & 2.871237746 & -0.152454565 \\
$\mathrm{C}$ & -0.380060976 & 3.914334167 & 0.028250530 \\
$\mathrm{O}$ & 0.263414175 & 4.165996050 & 1.022648485 \\
$\mathrm{O}$ & -0.166745900 & 4.644675181 & -1.101276452 \\
$\mathrm{H}$ & -1.087184285 & 1.559361792 & 1.503322430 \\
$\mathrm{H}$ & -2.143627524 & 2.946856053 & 1.889474464 \\
$\mathrm{H}$ & -1.008031236 & 2.070935567 & -0.801004260 \\
$\mathrm{H}$ & -2.271190248 & 3.300390154 & -0.714328803 \\
$\mathrm{Pd}$ & -2.960762489 & -0.555396992 & -0.606004109 \\
$\mathrm{Cl}$ & -5.308845576 & -0.786550415 & -0.694799157 \\
$\mathrm{Cl}$ & -2.664103198 & -2.388173236 & -2.117317625 \\
$\mathrm{H}$ & 0.544445400 & 5.252210210 & -0.850822756 \\
$\mathrm{Cl}$ & -0.601702524 & -0.301051576 & -0.443067183
\end{tabular}

$\left[\mathrm{PdCl}_{3}\left(\mathrm{~S}-\mathrm{H}_{2} \mathrm{MPA}\right)\right]^{-} \mathrm{SMD}$

$\begin{array}{llll}\mathrm{S} & -3.315369507 & 1.069108801 & 1.065060585 \\ \mathrm{C} & -1.860439742 & 2.159783231 & 1.134498973\end{array}$




$\begin{array}{lr}\mathrm{C} & -1.580698073 \\ \mathrm{C} & -0.507115101 \\ \mathrm{O} & 0.348080670 \\ \mathrm{O} & -0.572206773 \\ \mathrm{H} & -0.967056813 \\ \mathrm{H} & -2.089368081 \\ \mathrm{H} & -1.253843721 \\ \mathrm{H} & -2.494015185 \\ \mathrm{Pd} & -2.928037621 \\ \mathrm{Cl} & -5.252463390 \\ \mathrm{Cl} & -2.651195195 \\ \mathrm{H} & 0.164577175 \\ \mathrm{Cl} & -0.614788717\end{array}$

2.829244013

3.869802463

3.984739720

4.696713884

1.607014606

2.919882811

2.055170904

3.277497929

$-0.527725061$

$-0.597836914$

$-2.202073286$

5.332707554

$-0.529179279$

$\left[\mathrm{PdCl}_{3}\left(\mathrm{~S}-\mathrm{H}_{3} \mathrm{CSA}\right)\right]$

$\mathrm{S}$

$\mathrm{O}$

$\mathrm{O}$

$\mathrm{N}$

$\mathrm{C}$

C

C

\author{
C
}

$\mathrm{H}$

$\mathrm{H}$

$\mathrm{H}$

$\mathrm{H}$
$\mathrm{H}$

H

$\mathrm{H}$

$\mathrm{H}$

$\mathrm{H}$

$\mathrm{P}$

$\mathrm{Cl}$

$\begin{array}{rr}\mathrm{Cl} & 2.258627266 \\ \mathrm{Cl} & -0.840789757\end{array}$

O $\quad-2.247662045$

O $\quad-0.627708288$

H $\quad-2.105994203$
0.427039473

5.578320303

5.345495083

3.147278979

4.914730679

3.451040079

3.055933223

1.606986258

2.859701036

3.700720097

3.276903605

1.409253628

1.300272161

3.493329347

2.063729260

3.629935390

6.268892965

$-1.225713137$

$-2.923339930$

0.292553006

$-2.682450540$

$-0.201150734$

1.244394604

$-1.170775020$
$-0.197846115$

$-0.161360411$

0.689610449

$-1.219987321$

1.458431964

1.901117373

$-0.916415990$

$-0.618129134$

$-0.569452444$

$-0.885952444$

$-2.268392263$

$-1.175682024$

$-0.112546824$

$-0.513848923$

$-0.417219252$

$-1.214665036$

0.603680628

$-0.617174886$

$-0.230352631$

0.431491122

0.880854544

$-1.158850890$

1.308403133

$-0.299848730$

1.326418532

1.594379664

1.562129239

0.656731556

0.218865478

$-1.480815399$

$-0.121669174$

0.205002873

0.906335402

$-1.009071530$

$-0.423726862$

$-1.738278032$

$-0.673643671$

$\left[\mathrm{PdCl}_{3}\left(\mathrm{~S}-\mathrm{H}_{3} \mathrm{CSA}\right)\right] \mathrm{SMD}$

$\mathrm{S}$

O

$\mathrm{O}$

$\mathrm{N}$

C

C

\section{c}

\section{c}

$\stackrel{\mathrm{H}}{\mathrm{H}}$

$\mathrm{H}$

$\mathrm{H}$
0.382831791

5.751240445

5.399749400

3.204348150

5.024464065

3.530095454

3.032985002

1.577409601

3.038569388

3.633895722

3.231219353
$-0.530556849$

$-0.194081805$

$-1.135956336$

0.503157362

$-0.516481542$

$-0.262406372$

0.398029564

0.827674742

$-1.240785951$

1.295011835

$-0.309884363$ 


$\begin{array}{lrrr}\mathrm{H} & -1.748816532 & 1.303382646 & 1.318534492 \\ \mathrm{H} & 0.010004930 & 1.316011160 & 1.517366706 \\ \mathrm{H} & 1.745356998 & 3.600665570 & 1.447080767 \\ \mathrm{H} & 1.864552160 & 2.168529316 & 0.582659068 \\ \mathrm{H} & 2.553462409 & 3.579937785 & 0.033295006 \\ \mathrm{H} & -0.648451461 & 6.365595183 & -1.278458546 \\ \mathrm{Pd} & 0.630766398 & -1.401399528 & 0.037181768 \\ \mathrm{Cl} & 1.960191865 & -3.199597310 & 0.566824540 \\ \mathrm{Cl} & 2.255875203 & 0.096138956 & 0.840404366 \\ \mathrm{Cl} & -1.052197556 & -2.783741550 & -0.800214624 \\ \mathrm{O} & -2.176659832 & -0.050221072 & -0.679809789 \\ \mathrm{O} & -0.201593710 & 1.092734475 & -1.753696123 \\ \mathrm{H} & -2.164764374 & -1.031764187 & -0.869760762 \\ & & & \\ & & & \\ \mathrm{~S} & 7.191610780 & 0.182926283 & 2.871241922 \\ \mathrm{C} & 7.519686150 & -1.447031057 & 2.148105331 \\ \mathrm{C} & 8.761381183 & -2.100208459 & 2.732212430 \\ \mathrm{~N} & 9.883528659 & -1.138280985 & 2.902581101 \\ \mathrm{H} & 7.612533431 & -1.227323574 & 1.072236010 \\ \mathrm{H} & 6.634031453 & -2.073949742 & 2.322350794 \\ \mathrm{H} & 9.076048207 & -2.926289797 & 2.079794126 \\ \mathrm{H} & 8.550766753 & -2.499213163 & 3.736339478 \\ \mathrm{H} & 10.803653018 & -1.565267038 & 2.788603498 \\ \mathrm{H} & 9.779691629 & -0.751116084 & 3.922713670 \\ \mathrm{H} & 9.770632205 & -0.298501257 & 2.306459053 \\ \mathrm{O} & 8.324906045 & 0.995742651 & 2.361682479 \\ \mathrm{O} & 5.868199666 & 0.545442808 & 2.069359510 \\ \mathrm{H} & 5.146771554 & 0.609620575 & 2.788867823 \\ \mathrm{Pd} & 6.766169327 & -0.027847945 & 5.091717218 \\ \mathrm{Cl} & 6.271303801 & -0.353026477 & 7.279958866 \\ \mathrm{Cl} & 4.519868832 & 0.325247037 & 4.600283536 \\ \mathrm{Cl} & 9.053944853 & -0.382786112 & 5.593975981 \\ & & & \end{array}$

$\left[\mathrm{PdCl}_{3}\left(\mathrm{~S}-\mathrm{HCysteO}_{2} \mathrm{H}\right)\right] \mathrm{SMD}$

$\begin{array}{lrrr}\mathrm{S} & 7.132766648 & 0.089212803 & 2.962438220 \\ \mathrm{C} & 7.570888257 & -1.461550217 & 2.151924518 \\ \mathrm{C} & 8.837287722 & -2.123229494 & 2.659317795 \\ \mathrm{~N} & 9.957574375 & -1.167517627 & 2.838947133 \\ \mathrm{H} & 7.647422163 & -1.158541503 & 1.093313824 \\ \mathrm{H} & 6.712186197 & -2.141165771 & 2.261978203 \\ \mathrm{H} & 9.129249148 & -2.895105323 & 1.934781318 \\ \mathrm{H} & 8.675974305 & -2.610658063 & 3.630556217 \\ \mathrm{H} & 10.888221898 & -1.587375042 & 2.756154743 \\ \mathrm{H} & 9.868872392 & -0.757227378 & 3.798465383 \\ \mathrm{H} & 9.909818096 & -0.367732567 & 2.194961569 \\ \mathrm{O} & 8.203229706 & 1.042858675 & 2.591433097 \\ \mathrm{O} & 5.815733323 & 0.402937458 & 2.105325955 \\ \mathrm{H} & 5.090705818 & 0.599972743 & 2.770586674 \\ \mathrm{Pd} & 6.635089331 & -0.002573394 & 5.175672912 \\ \mathrm{Cl} & 6.159227834 & -0.042489042 & 7.420464429 \\ \mathrm{Cl} & 4.428651439 & 0.569509876 & 4.681140040\end{array}$




\begin{tabular}{lrrr}
\multicolumn{4}{c}{$\left[\mathrm{PdCl}_{3}\left(\mathrm{~S}_{-} \mathrm{H}_{2} \mathrm{CysO}_{2} \mathrm{H}\right)\right]$} \\
$\mathrm{S}$ & 0.536932564 & -0.265768946 & 0.576783568 \\
$\mathrm{O}$ & 4.304827246 & -1.220734634 & -0.401011630 \\
$\mathrm{O}$ & 3.870113881 & -1.427281474 & 1.793763725 \\
$\mathrm{~N}$ & 3.062111683 & 1.073404707 & -0.650885621 \\
$\mathrm{C}$ & 3.840601788 & -0.813913206 & 0.630477536 \\
$\mathrm{C}$ & 3.195354342 & 0.566985115 & 0.735250279 \\
$\mathrm{C}$ & 1.858477808 & 0.601626804 & 1.461252464 \\
$\mathrm{H}$ & 2.034321324 & 0.701431817 & -0.971595045 \\
$\mathrm{H}$ & 3.781342811 & 0.627573619 & -1.236938615 \\
$\mathrm{H}$ & 3.108542752 & 2.091448855 & -0.715783381 \\
$\mathrm{H}$ & 3.907752522 & 1.193207007 & 1.300066395 \\
$\mathrm{H}$ & 4.257801836 & -2.309881912 & 1.675117573 \\
$\mathrm{H}$ & 1.447053410 & 1.620871607 & 1.570550832 \\
$\mathrm{H}$ & 1.930583006 & 0.137167191 & 2.453363955 \\
$\mathrm{O}$ & 1.019132206 & -1.772895644 & 0.737799298 \\
$\mathrm{O}$ & 0.773100700 & 0.143632227 & -0.862049016 \\
$\mathrm{H}$ & 0.164216574 & -2.263120821 & 1.064352036 \\
$\mathrm{Pd}$ & -1.434413269 & 0.048986908 & 1.549976248 \\
$\mathrm{Cl}$ & -3.401940235 & 0.413511268 & 2.602580022 \\
$\mathrm{Cl}$ & -1.620399880 & -2.299141506 & 1.694772657 \\
$\mathrm{Cl}$ & -0.948538608 & 2.319434154 & 1.357280436
\end{tabular}

$\left[\mathrm{PdCl}_{3}\left(\mathrm{~S}-\mathrm{H}_{2} \mathrm{CysO}_{2} \mathrm{H}\right)\right] \mathrm{SMD}$

$\begin{array}{lrrr}\mathrm{S} & 0.518834453 & -0.236113009 & 0.553247963 \\ \mathrm{O} & 4.185301418 & -1.331599216 & -0.428592140 \\ \mathrm{O} & 3.835600962 & -1.449926010 & 1.794539063 \\ \mathrm{~N} & 3.148595370 & 1.107738607 & -0.653093368 \\ \mathrm{C} & 3.786141274 & -0.873062534 & 0.611258394 \\ \mathrm{C} & 3.203930225 & 0.531478321 & 0.713416955 \\ \mathrm{C} & 1.854541713 & 0.618492401 & 1.414719079 \\ \mathrm{H} & 2.283293476 & 0.769208148 & -1.127560197 \\ \mathrm{H} & 3.954981488 & 0.795350241 & -1.213468307 \\ \mathrm{H} & 3.149142785 & 2.133127258 & -0.647771757 \\ \mathrm{H} & 3.911292250 & 1.124404462 & 1.314774455 \\ \mathrm{H} & 4.205378532 & -2.352853873 & 1.745085834 \\ \mathrm{H} & 1.500242932 & 1.656379010 & 1.511219200 \\ \mathrm{H} & 1.896668419 & 0.169233811 & 2.416668294 \\ \mathrm{O} & 1.019575880 & -1.749038953 & 0.689873206 \\ \mathrm{O} & 0.628982803 & 0.164420029 & -0.876698254 \\ \mathrm{H} & 0.249406915 & -2.266430980 & 1.087431950 \\ \mathrm{Pd} & -1.454003425 & 0.020402333 & 1.607700245 \\ \mathrm{Cl} & -3.451463849 & 0.346075170 & 2.688338858 \\ \mathrm{Cl} & -1.590954974 & -2.309104646 & 1.869956811 \\ \mathrm{Cl} & -1.148514184 & 2.298362565 & 1.294077430\end{array}$

$\left[\mathrm{PdCl}_{3}\left(\mathrm{~S}-\mathrm{H}_{2} \mathrm{SPA}\right)\right]^{2-}$

$\begin{array}{llll}\mathrm{S} & -1.690120430 & 0.739834314 & 0.261435554 \\ \mathrm{C} & -1.386076092 & 1.858000986 & 1.652229522 \\ \mathrm{C} & -1.079317092 & 3.253877163 & 1.163292472\end{array}$




$\begin{array}{lrrr}\mathrm{C} & -1.142034722 & 4.266792332 & 2.262655960 \\ \mathrm{O} & -1.472173580 & 4.064178198 & 3.404327574 \\ \mathrm{O} & -0.784529142 & 5.488776682 & 1.834003264 \\ \mathrm{H} & -0.562507472 & 1.415264800 & 2.228902276 \\ \mathrm{H} & -2.311629900 & 1.823485550 & 2.244816908 \\ \mathrm{H} & -0.084784870 & 3.279928959 & 0.687850285 \\ \mathrm{H} & -1.797184441 & 3.545691607 & 0.378312226 \\ \mathrm{Pd} & 0.105162523 & -0.475416865 & -0.424169919 \\ \mathrm{Cl} & -1.348606794 & -2.335384576 & -0.549089092 \\ \mathrm{Cl} & 1.897983574 & -1.725726889 & -1.118334261 \\ \mathrm{H} & -0.855278228 & 6.069539656 & 2.606168127 \\ \mathrm{O} & -2.634871938 & -0.285898177 & 1.084150712 \\ \mathrm{H} & -2.440216383 & -1.175773507 & 0.655897226 \\ \mathrm{O} & -2.478493468 & 1.499407791 & -0.727301856 \\ \mathrm{Cl} & 1.435696416 & 1.415471344 & -0.142490332\end{array}$

$\left[\mathrm{PdCl}_{3}\left(\mathrm{~S}-\mathrm{H}_{2} \mathrm{SPA}\right)\right]^{2-} \mathrm{SMD}$

$\begin{array}{lrrr}\mathrm{S} & -1.676929134 & 0.734841333 & 0.262794864 \\ \mathrm{C} & -1.441944477 & 1.874546505 & 1.638657125 \\ \mathrm{C} & -1.078184198 & 3.264525667 & 1.170824721 \\ \mathrm{C} & -1.122975452 & 4.249862157 & 2.300689737 \\ \mathrm{O} & -1.473876167 & 3.998454556 & 3.429993420 \\ \mathrm{O} & -0.726630561 & 5.461923252 & 1.904817617 \\ \mathrm{H} & -0.688614617 & 1.415437345 & 2.295251208 \\ \mathrm{H} & -2.413441143 & 1.863061557 & 2.155623028 \\ \mathrm{H} & -0.077878673 & 3.283255077 & 0.713821684 \\ \mathrm{H} & -1.780242879 & 3.610716280 & 0.395243309 \\ \mathrm{Pd} & 0.122178105 & -0.481823321 & -0.411077681 \\ \mathrm{Cl} & -1.243757294 & -2.396431151 & -0.378015242 \\ \mathrm{Cl} & 1.935547872 & -1.735064280 & -1.095640570 \\ \mathrm{H} & -0.787526672 & 6.092383604 & 2.645750337 \\ \mathrm{O} & -2.661557224 & -0.274860526 & 1.048231484 \\ \mathrm{H} & -2.458898048 & -1.183339085 & 0.676372112 \\ \mathrm{O} & -2.410780954 & 1.459050219 & -0.797394565 \\ \mathrm{Cl} & 1.356529478 & 1.485510178 & -0.453285944\end{array}$

$\left[\mathrm{PdCl}_{3}\left(\mathrm{~S}-\mathrm{H}_{2} \mathrm{DTPDA}\right)\right]$

$\begin{array}{rrrr}\mathrm{S} & 1.479523782 & 1.024284972 & 3.450065849 \\ \mathrm{C} & 2.395606071 & -0.263263628 & 2.529387591 \\ \mathrm{C} & 3.871811707 & 0.030313826 & 2.403028512 \\ \mathrm{C} & 4.187973611 & 1.160410080 & 1.460964528 \\ \mathrm{O} & 5.394937862 & 1.696996881 & 1.709374569 \\ \mathrm{O} & 3.492244416 & 1.540907890 & 0.557078784 \\ \mathrm{H} & 1.902101767 & -0.253902451 & 1.544700938 \\ \mathrm{H} & 2.175144346 & -1.232098775 & 2.999665072 \\ \mathrm{H} & 4.325492587 & 0.231851228 & 3.386265332 \\ \mathrm{H} & 4.389769073 & -0.860805946 & 2.005124220 \\ \mathrm{H} & 5.514811625 & 2.393967621 & 1.046499027 \\ \mathrm{~S} & 2.509134936 & 1.099000326 & 5.271888560 \\ \mathrm{C} & 1.707649175 & -0.202939660 & 6.272272511 \\ \mathrm{C} & 0.518852130 & 0.323512852 & 7.047592780 \\ \mathrm{H} & 2.501694505 & -0.552004921 & 6.950778168\end{array}$




$\begin{array}{rrrr}\mathrm{H} & 1.408940940 & -1.025953161 & 5.603155238 \\ \mathrm{C} & 0.916351455 & 1.207795948 & 8.190073000 \\ \mathrm{H} & -0.169472286 & 0.885774321 & 6.388927447 \\ \mathrm{H} & -0.090250641 & -0.507589167 & 7.442352203 \\ \mathrm{O} & -0.145384350 & 1.725794342 & 8.823027116 \\ \mathrm{O} & 2.046911601 & 1.444027915 & 8.541733781 \\ \mathrm{H} & 0.213223668 & 2.291818781 & 9.522328511 \\ \mathrm{Pd} & -0.679149859 & 0.207132687 & 3.892247677 \\ \mathrm{Cl} & -2.837821199 & -0.442816783 & 4.310435097 \\ \mathrm{Cl} & -0.006397900 & -2.038990549 & 3.734043775 \\ \mathrm{Cl} & -1.196787458 & 2.439508430 & 4.276867698\end{array}$

$\left[\mathrm{PdCl}_{3}\left(\mathrm{~S}-\mathrm{H}_{2} \mathrm{DTPDA}\right)\right]^{-} \mathrm{SMD}$

$\begin{array}{lrrr}\mathrm{S} & 1.661488661 & 1.090391139 & 3.618460009 \\ \mathrm{C} & 2.505931524 & -0.284488644 & 2.766998489 \\ \mathrm{C} & 3.928064305 & 0.074641454 & 2.410613500 \\ \mathrm{C} & 4.013857592 & 1.141411006 & 1.354479070 \\ \mathrm{O} & 5.258674871 & 1.606287633 & 1.215348871 \\ \mathrm{O} & 3.082426971 & 1.535598865 & 0.692178550 \\ \mathrm{H} & 1.890850898 & -0.446293199 & 1.868731034 \\ \mathrm{H} & 2.426954855 & -1.187482698 & 3.384704645 \\ \mathrm{H} & 4.505175168 & 0.391178364 & 3.294333703 \\ \mathrm{H} & 4.447885601 & -0.818404658 & 2.021821677 \\ \mathrm{H} & 5.277149320 & 2.293277118 & 0.524263032 \\ \mathrm{~S} & 2.587068535 & 1.132996803 & 5.483843214 \\ \mathrm{C} & 1.878608647 & -0.284528707 & 6.388405601 \\ \mathrm{C} & 0.590252810 & 0.037459364 & 7.108547060 \\ \mathrm{H} & 2.674009533 & -0.564921494 & 7.096218509 \\ \mathrm{H} & 1.728243933 & -1.118537344 & 5.688143321 \\ \mathrm{C} & 0.757347812 & 1.081278652 & 8.172604057 \\ \mathrm{H} & -0.204054817 & 0.344102542 & 6.406917168 \\ \mathrm{H} & 0.202927010 & -0.871296109 & 7.603192607 \\ \mathrm{O} & -0.415103746 & 1.589320245 & 8.566729419 \\ \mathrm{O} & 1.816600756 & 1.425157479 & 8.645840575 \\ \mathrm{H} & -0.268040330 & 2.233027016 & 9.283699675 \\ \mathrm{Pd} & -0.578229230 & 0.577731915 & 3.884830097 \\ \mathrm{Cl} & -2.851857636 & 0.189629884 & 4.089381103 \\ \mathrm{Cl} & -0.155230880 & -1.719081180 & 3.955580775 \\ \mathrm{Cl} & -0.934090599 & 2.874277611 & 3.834012225\end{array}$

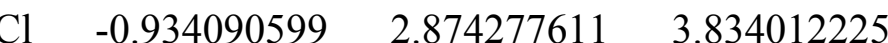

\begin{tabular}{lrrr} 
& \multicolumn{4}{c}{$\left[\mathrm{Pd}_{2} \mathrm{Cl}_{6}\left(\mathrm{~S}, \mathrm{~S}^{\prime}-\mathrm{H}_{2} \mathrm{DTPDA}\right)\right]^{2-}$} \\
$\mathrm{S}$ & 1.354388039 & 0.662253818 & 3.665416729 \\
$\mathrm{C}$ & 2.294786039 & -0.083934231 & 2.280420110 \\
$\mathrm{C}$ & 3.784208103 & 0.203585952 & 2.269431917 \\
$\mathrm{C}$ & 4.155797278 & 1.674106529 & 2.266203145 \\
$\mathrm{O}$ & 5.431690459 & 1.845448956 & 2.604139883 \\
$\mathrm{O}$ & 3.390033498 & 2.559975094 & 1.985575561 \\
$\mathrm{H}$ & 1.789840564 & 0.367844726 & 1.412054377 \\
$\mathrm{H}$ & 2.090627237 & -1.164886662 & 2.312260512 \\
$\mathrm{H}$ & 4.303053890 & -0.275584193 & 3.117445338 \\
$\mathrm{H}$ & 4.218982698 & -0.259023509 & 1.364833941 \\
$\mathrm{H}$ & 5.544675027 & 2.675112309 & 3.123448437
\end{tabular}




$\begin{array}{lrrr}\mathrm{S} & 2.630347081 & -0.017332244 & 5.257217580 \\ \mathrm{C} & 1.380285252 & -0.252938084 & 6.552271140 \\ \mathrm{C} & 0.546826124 & 0.937701069 & 6.972926597 \\ \mathrm{H} & 1.985995793 & -0.619113133 & 7.397121147 \\ \mathrm{H} & 0.767358903 & -1.078004599 & 6.156274881 \\ \mathrm{C} & 1.221399499 & 1.787416301 & 8.011630376 \\ \mathrm{H} & 0.229357695 & 1.569526563 & 6.128962261 \\ \mathrm{H} & -0.395813573 & 0.566531865 & 7.412459333 \\ \mathrm{O} & 0.539196729 & 2.914210520 & 8.258456580 \\ \mathrm{O} & 2.208390322 & 1.486691359 & 8.636541553 \\ \mathrm{H} & 1.110962206 & 3.432673658 & 8.842339855 \\ \mathrm{Pd} & -0.583228025 & -0.618140649 & 3.826196104 \\ \mathrm{Cl} & -2.573806611 & -1.791728787 & 3.952109465 \\ \mathrm{Cl} & 0.661055686 & -2.602562966 & 3.790574192 \\ \mathrm{Cl} & -1.694697476 & 1.420523350 & 3.967138654 \\ \mathrm{Pd} & 4.094318078 & 1.740132021 & 5.532650292 \\ \mathrm{Cl} & 5.728317546 & 3.408250783 & 5.279137076 \\ \mathrm{Cl} & 2.430668170 & 3.351572832 & 5.622574480 \\ \mathrm{Cl} & 5.704827729 & 0.037322394 & 5.546898873\end{array}$

\begin{tabular}{|c|c|c|c|}
\hline \multicolumn{4}{|c|}{$\left[\mathrm{Pd}_{2} \mathrm{Cl}_{6}\left(\mathrm{~S}, \mathrm{~S}^{\prime}-\mathrm{H}_{2} \mathrm{DTPDA}\right)\right]^{2-} \mathrm{SMD}$} \\
\hline $\mathrm{S}$ & 1.346424445 & 0.630967034 & 3.6293 \\
\hline $\mathrm{C}$ & 2.304326491 & -0.115856561 & \\
\hline $\mathrm{C}$ & 3.787150784 & 0.192370618 & 2.275678671 \\
\hline $\mathrm{C}$ & 4.134062367 & 1.657962896 & 2.314213973 \\
\hline $\mathrm{O}$ & 5.406723592 & 1.843037122 & 2.66 \\
\hline $\mathrm{O}$ & 3.367034457 & & \\
\hline $\mathrm{H}$ & 1.807899502 & 0.308337744 & 1.3 \\
\hline $\mathrm{H}$ & 2.124154975 & 312120 & \\
\hline $\mathrm{H}$ & 4.300 & -0.3 & \\
\hline $\mathrm{H}$ & 4.236193031 & -0.2 & \\
\hline $\mathrm{H}$ & 5.536500121 & & \\
\hline S & 2.597847412 & -0.02 & 5.2 \\
\hline $\mathrm{C}$ & 1.35 & & \\
\hline $\mathrm{C}$ & & & \\
\hline $\mathrm{H}$ & $\$ 1871463$ & -0.7 & 7.3 \\
\hline $\mathrm{H}$ & 0.709 & -1.0 & \\
\hline $\mathrm{C}$ & 1.3 & & \\
\hline $\mathrm{H}$ & & & \\
\hline $\mathrm{H}$ & -0.320364959 & 0.5 & 7.5 \\
\hline $\mathrm{O}$ & 0.646466697 & 2.9 & \\
\hline $\mathrm{O}$ & 2.419370622 & 1.54 & 8.4 \\
\hline $\mathrm{H}$ & 1.174510645 & 6193 & 8.8 \\
\hline $\mathrm{Pd}$ & -0.594784746 & 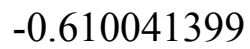 & \\
\hline $\mathrm{Cl}$ & -2.608236932 & -1.76 & \\
\hline $\mathrm{Cl}$ & 0.630773483 & -2.595068263 & 3.77307705 \\
\hline $\mathrm{Cl}$ & -1.685168724 & 1.442222049 & 3.943 \\
\hline $\mathrm{Pd}$ & 4.027597241 & & \\
\hline $\mathrm{Cl}$ & 5.644159842 & 3.438311366 & 5.54222594 \\
\hline $\mathrm{Cl}$ & 2.334787401 & 3.341786867 & 5.42135378 \\
\hline $\mathrm{Cl}$ & 5.658089354 & 0.097787134 & 5.73808250 \\
\hline
\end{tabular}




\begin{tabular}{|c|c|c|c|}
\hline \multicolumn{4}{|c|}{$\left[\mathrm{PdCl}_{3}\left(\mathrm{~S}-\mathrm{H}_{4} \mathrm{HCysS}\right)\right]^{+}$} \\
\hline $\mathrm{S}$ & 5.277028854 & 1.865746217 & 0.227730420 \\
\hline $\mathrm{S}$ & 6.108450583 & 1.931584580 & 2.154973988 \\
\hline $\mathrm{O}$ & 1.056322299 & 4.367412849 & 2.668375637 \\
\hline $\mathrm{O}$ & 1.813878835 & 2.764256696 & 4.048317768 \\
\hline $\mathrm{O}$ & 11.241530835 & 5.217053665 & 1.542216317 \\
\hline $\mathrm{O}$ & 10.954875638 & 3.767492301 & -0.145949030 \\
\hline $\mathrm{N}$ & 2.782798667 & 3.894344885 & 155546 \\
\hline $\mathrm{N}$ & 9.495855671 & 4.081292431 & 75523 \\
\hline $\mathrm{C}$ & 1.800856884 & & \\
\hline $\mathrm{C}$ & 2.886586416 & 3.005073601 & 6120 \\
\hline $\mathrm{C}$ & 2.832939207 & 1.502335098 & \\
\hline $\mathrm{C}$ & 3.745164205 & 0.949507946 & 4646 \\
\hline $\mathrm{C}$ & 0738770 & & \\
\hline $\mathrm{C}$ & 9.835097480 & & 6946 \\
\hline $\mathrm{C}$ & 8.637044425 & 2.775 & \\
\hline $\mathrm{C}$ & 17702 & & 1967 \\
\hline $\mathrm{H}$ & 8248 & & \\
\hline $\mathrm{H}$ & 86539 & & 7344 \\
\hline $\mathrm{H}$ & 91247 & & \\
\hline $\mathrm{H}$ & 3.96 & & \\
\hline $\mathrm{H}$ & 4741 & & \\
\hline $\mathrm{H}$ & 37623 & & \\
\hline $\mathrm{H}$ & 3048 & & \\
\hline $\mathrm{H}$ & 9.0 & & \\
\hline $\mathrm{H}$ & & & \\
\hline $\mathrm{H}$ & 4935 & & 909 \\
\hline $\mathrm{H}$ & 0121 & & \\
\hline $\mathrm{H}$ & 06065 & & \\
\hline $\mathrm{H}$ & 49022 & & -0 . \\
\hline $\mathrm{H}$ & 00907 & & \\
\hline $\mathrm{H}$ & 5870 & & \\
\hline $\mathrm{H}$ & 11.5 & & -0 \\
\hline $\mathrm{H}$ & 33068 & & 951 \\
\hline $\mathrm{Pd}$ & 52232 & & \\
\hline $\mathrm{Cl}$ & 1463 & & \\
\hline $\mathrm{Cl}$ & 97645 & & 609 \\
\hline $\mathrm{Cl}$ & 69243 & & 820 \\
\hline $\mathrm{H}$ & 10.347317453 & & 3.520098129 \\
\hline \multicolumn{4}{|c|}{$\left[\mathrm{PdCl}_{3}\left(\mathrm{~S}-\mathrm{H}_{4} \mathrm{HCysS}\right)\right]^{+} \mathrm{SMD}$} \\
\hline S & 5.294289847 & & \\
\hline $\mathrm{S}$ & 625465 & & \\
\hline $\mathrm{O}$ & 0.932601725 & & 2.712288973 \\
\hline $\mathrm{O}$ & 1.981268544 & 5164 & 4147 \\
\hline $\mathrm{O}$ & 11.344608659 & & \\
\hline $\mathrm{O}$ & 10.999478653 & 3.680539878 & -0.154186072 \\
\hline $\mathrm{N}$ & 2.618013362 & 3.868102900 & 0.709449163 \\
\hline $\mathrm{N}$ & 9.535700314 & 4.044934175 & 3.109098636 \\
\hline $\mathrm{C}$ & & & \\
\hline $\mathrm{C}$ & 2.830454960 & 3.018713667 & 1.905258710 \\
\hline $\mathrm{C}$ & 2.862192841 & 1.509544074 & 1.665381356 \\
\hline
\end{tabular}




$\begin{array}{crrr}\mathrm{C} & 3.786385354 & 1.005893333 & 0.555557618 \\ \mathrm{C} & 10.811739291 & 4.155404460 & 1.063021818 \\ \mathrm{C} & 9.864596433 & 3.287478887 & 1.879899547 \\ \mathrm{C} & 8.639061048 & 2.794245857 & 1.114376611 \\ \mathrm{C} & 7.865723041 & 1.671673881 & 1.822801660 \\ \mathrm{H} & 3.795397932 & 3.344154019 & 2.328436675 \\ \mathrm{H} & 3.174257377 & 1.070707200 & 2.624420337 \\ \mathrm{H} & 1.850164966 & 1.116626358 & 1.478567979 \\ \mathrm{H} & 4.042075720 & -0.048065056 & 0.736767548 \\ \mathrm{H} & 3.304362256 & 1.038563563 & -0.437054375 \\ \mathrm{H} & 10.472339095 & 2.416805284 & 2.189211536 \\ \mathrm{H} & 7.972357492 & 3.641832071 & 0.888575952 \\ \mathrm{H} & 9.003731091 & 2.423022232 & 0.147993861 \\ \mathrm{H} & 7.865883403 & 0.732511654 & 1.251267470 \\ \mathrm{H} & 8.255288580 & 1.435339843 & 2.825899052 \\ \mathrm{H} & 1.801275123 & 4.478655334 & 0.889094654 \\ \mathrm{H} & 3.465701426 & 4.464870307 & 0.592029237 \\ \mathrm{H} & 2.456165517 & 3.356207347 & -0.162267776 \\ \mathrm{H} & 8.905013883 & 4.853187360 & 2.952365233 \\ \mathrm{H} & 9.011468261 & 3.480109607 & 3.807719774 \\ \mathrm{H} & 11.665597761 & 4.209183984 & -0.635023585 \\ \mathrm{H} & 1.337514100 & 3.055339596 & 4.759478450 \\ \mathrm{Pd} & 6.286649542 & 4.191234648 & 2.974665619 \\ \mathrm{Cl} & 7.084104503 & 6.208670980 & 3.793869330 \\ \mathrm{Cl} & 7.120650226 & 3.044410962 & 4.836927704 \\ \mathrm{Cl} & 5.457902727 & 5.263323689 & 1.086195393 \\ \mathrm{H} & 10.371117985 & 4.444736738 & 3.552471758\end{array}$

\begin{tabular}{lrrr} 
& \multicolumn{4}{c}{$\left[\mathrm{Pd}_{2} \mathrm{Cl}_{6}\left(\mathrm{~S}, \mathrm{~S}^{\prime}-\mathrm{H}_{4} \mathrm{HCySS}\right)\right]^{0}$} \\
$\mathrm{~S}$ & 5.176088511 & 2.055671350 & 0.399216025 \\
$\mathrm{~S}$ & 5.995154461 & 2.112398859 & 2.387005271 \\
$\mathrm{O}$ & 0.699159114 & 4.101160408 & 2.595317777 \\
$\mathrm{O}$ & 1.565068574 & 2.658951354 & 4.079113322 \\
$\mathrm{O}$ & 11.323540043 & 4.658507790 & 0.891416082 \\
$\mathrm{O}$ & 11.026569534 & 2.694207030 & -0.152584492 \\
$\mathrm{~N}$ & 2.602458303 & 3.770852432 & 0.825915983 \\
$\mathrm{~N}$ & 9.527674781 & 4.209568016 & 2.729792341 \\
$\mathrm{C}$ & 1.535454726 & 3.300745486 & 2.929231845 \\
$\mathrm{C}$ & 2.707415343 & 2.925515378 & 2.038601271 \\
$\mathrm{C}$ & 2.791536257 & 1.413670385 & 1.793479998 \\
$\mathrm{C}$ & 3.770307897 & 0.944295830 & 0.715988816 \\
$\mathrm{C}$ & 10.803504574 & 3.576682001 & 0.796088733 \\
$\mathrm{C}$ & 9.811177072 & 3.060911091 & 1.836875590 \\
$\mathrm{C}$ & 8.584929536 & 2.415505595 & 1.200318264 \\
$\mathrm{C}$ & 7.709305924 & 1.543340762 & 2.108188363 \\
$\mathrm{H}$ & 3.616506240 & 3.268774916 & 2.563162312 \\
$\mathrm{H}$ & 3.069568596 & 0.977104931 & 2.763338738 \\
$\mathrm{H}$ & 1.792458158 & 1.014039270 & 1.552124452 \\
$\mathrm{H}$ & 4.176126466 & -0.062792062 & 0.913279967 \\
$\mathrm{H}$ & 3.304767507 & 0.896124013 & -0.284217119 \\
$\mathrm{H}$ & 10.352056789 & 2.312339403 & 2.442485379 \\
$\mathrm{H}$ & 8.006527626 & 3.181622939 & 0.655110281
\end{tabular}




$\begin{array}{crrr}\mathrm{H} & 8.964778647 & 1.766969462 & 0.396649783 \\ \mathrm{H} & 7.577921482 & 0.524852510 & 1.701933387 \\ \mathrm{H} & 8.079555037 & 1.469576102 & 3.142566654 \\ \mathrm{H} & 1.763914130 & 4.368700754 & 0.934218956 \\ \mathrm{H} & 3.493095753 & 4.384583519 & 0.791614171 \\ \mathrm{H} & 2.536700719 & 3.239788658 & -0.045375609 \\ \mathrm{H} & 8.883001628 & 4.915922421 & 2.321756181 \\ \mathrm{H} & 9.038209037 & 3.922115467 & 3.616448912 \\ \mathrm{H} & 11.629823835 & 3.072721313 & -0.812235432 \\ \mathrm{H} & 0.812643372 & 2.950135290 & 4.619866493 \\ \mathrm{Pd} & 6.319548955 & 4.349018578 & 2.941641970 \\ \mathrm{Cl} & 7.269950666 & 6.399543161 & 3.379138474 \\ \mathrm{Cl} & 7.423237100 & 3.426696637 & 4.797212252 \\ \mathrm{Cl} & 5.105412896 & 5.342071561 & 1.217276387 \\ \mathrm{H} & 10.395146326 & 4.720431647 & 2.933574191 \\ \mathrm{Pd} & 6.681819772 & 0.977516505 & -0.951016768 \\ \mathrm{Cl} & 8.307442435 & 0.067674840 & -2.255411598 \\ \mathrm{Cl} & 7.024138420 & 3.094169781 & -1.853636391 \\ \mathrm{Cl} & 6.324730185 & -1.038489237 & 0.181404775\end{array}$

O

0
0
0

2.036002319 2.097037917

4.134625850

2.698391759

4.719566193

2.741146612

3.828131923

4.208095372

3.346329231

3.012597411

1.507445903

1.039040498

3.623740184

3.081727488

2.428074392

1.554131659

3.420025108

1.105883818

1.041410785

$-0.005530541$

1.088466268

2.329234196

3.183709003

1.787276494

0.528942006

1.480282164

4.406120629

4.469264300

3.297353570

4.843974222

3.881853756
0.271925981

2.239345307

2.881467029

4.193330498

1.093380399

0.032976993

0.812938758

2.739623340

3.071289812

2.008317198

1.781818512

0.617358803

0.946482667

1.861289289

1.119998538

1.995449141

2.392334321

2.720808949

1.665664102

0.759235385

$-0.355766794$

2.505038325

0.560412097

0.347785666

1.606823440

3.041514859

1.006931671

0.641129886

$-0.047406675$

2.245431504

3.591309511 


$\begin{array}{lr}\mathrm{H} & 12.022429923 \\ \mathrm{H} & 1.298127439 \\ \mathrm{Pd} & 6.151038061 \\ \mathrm{Cl} & 6.383258844 \\ \mathrm{Cl} & 7.193663452 \\ \mathrm{Cl} & 5.228271124 \\ \mathrm{H} & 10.246554985 \\ \mathrm{Pd} & 6.622940857 \\ \mathrm{Cl} & 8.006733599 \\ \mathrm{Cl} & 7.050189350 \\ \mathrm{Cl} & 6.208333442\end{array}$

S $\quad 0.893873333$

S $\quad-0.893873333$

O 4.823655352

O $\quad-4.823655352$

O 4.033746783

O $\quad-4.033746783$

$\mathrm{N} \quad 3.411245860$

$\mathrm{N} \quad-3.411245860$

C $\quad 4.155008177$

C $\quad-4.155008177$

C $\quad 3.338936246$

C $\quad-3.338936246$

C $\quad 1.910707145$

C $\quad-1.910707145$

$\mathrm{H} \quad 2.563338461$

$\mathrm{H} \quad-2.563338461$

$\mathrm{H} \quad 4.221248579$

$\mathrm{H} \quad-4.221248579$

$\mathrm{H} \quad 3.530337039$

$\mathrm{H} \quad-3.530337039$

$\mathrm{H} \quad 3.888093653$

$\mathrm{H} \quad-3.888093653$

$\mathrm{H} \quad 4.607632441$

$\mathrm{H} \quad-4.607632441$

$\mathrm{H} \quad 1.429495997$

$\mathrm{H} \quad-1.429495997$

$\mathrm{H} \quad 1.952810332$

H -1.952810332
3.075916578 2.928370958

4.320807670

6.429784350

3.363391059

5.252397404

4.771751529

0.755041846

$-0.436462509$

2.738158407

$-1.130311636$

$\mathrm{H}_{4} \mathrm{CysS}^{2+}$

$-0.514454432$

0.514454432

$-0.852922998$

0.852922998

$-1.510502193$

1.510502193

1.267508540

$-1.267508540$

$-0.699912818$

0.699912818

0.589842755

$-0.589842755$

0.403024716

$-0.403024716$

1.046704555

$-1.046704555$

0.824071313

$-0.824071313$

2.285817426

$-2.285817426$

1.202759106

$-1.202759106$

$-2.291027510$

2.291027510

1.375193921

$-1.375193921$

$-0.155626423$

0.155626423

$-0.524169702$

4.888649910

2.927439343

3.811932168

4.800542033

1.007613133

3.026719862

$-1.053884487$

$-2.465892782$

$-2.192330268$

0.262018264

$-0.104102817$

$-0.104102817$

$-0.549891552$

$-0.549891552$

1.455117264

1.455117264

$-0.701709355$

$-0.701709355$

0.437216453

0.437216453

0.623082925

0.623082925

1.126512212

1.126512212

$-1.252726113$

$-1.252726113$

$-1.197182375$

$-1.197182375$

$-0.653330671$

$-0.653330671$

1.358235722

1.358235722

1.342120699

1.342120699

1.314407896

1.314407896

2.072602238

2.072602238

$$
\mathrm{H}_{4} \mathrm{CysS}^{2+} \mathrm{SMD}
$$

S $\quad 0.878574327 \quad-0.434781095$

$-0.209840744$

$\mathrm{S} \quad-0.928002668$

0.538618730

$-0.136144877$

O $\quad 4.891606977$

$-0.866095649$

$-0.444665225$

O $\quad-4.885469848$

0.821619322

$-0.475762830$

$-1.626099863$

1.330571145

1.363093982

1.579738611

$-0.633043330$

1.354262780

$-0.619387801$

$-1.376005215$

0.428349348

$\mathrm{N}$ $-3.415042663$

$-0.726919276$ 


$\begin{array}{rrrr}\mathrm{C} & -4.103554141 & 0.689174501 & 0.432211750 \\ \mathrm{C} & 3.315210465 & 0.586825480 & 0.623249496 \\ \mathrm{C} & -3.320687209 & -0.602390411 & 0.643918984 \\ \mathrm{C} & 1.864598990 & 0.450300451 & 1.059469161 \\ \mathrm{C} & -1.879967571 & -0.429489426 & 1.103184272 \\ \mathrm{H} & 2.679320925 & 1.171511360 & -1.280602991 \\ \mathrm{H} & -2.704184704 & -1.038709610 & -1.287048821 \\ \mathrm{H} & 4.329543983 & 1.060888344 & -1.114154698 \\ \mathrm{H} & -4.328017689 & -1.194585538 & -1.072175867 \\ \mathrm{H} & 3.507444104 & 2.371719230 & -0.510766489 \\ \mathrm{H} & -3.309106706 & -2.389214060 & -0.516849152 \\ \mathrm{H} & 3.856509054 & 1.118640628 & 1.424465295 \\ \mathrm{H} & -3.868615258 & -1.168429370 & 1.418112211 \\ \mathrm{H} & 4.279055492 & -2.436002324 & 1.249642426 \\ \mathrm{H} & -4.335873041 & 2.406985486 & 1.237853739 \\ \mathrm{H} & 1.418917300 & 1.444280884 & 1.218286442 \\ \mathrm{H} & -1.391854128 & -1.407370998 & 1.226715919 \\ \mathrm{H} & 1.829039489 & -0.095450015 & 2.011877148 \\ \mathrm{H} & -1.864331508 & 0.090338882 & 2.070146558\end{array}$

$\begin{array}{cccc} & & \mathrm{H}_{2} \mathrm{Cyst}^{2+} & \\ \mathrm{S} & 8.088190198 & 1.079727423 & 1.883029174 \\ \mathrm{~S} & 6.300889741 & 0.236745436 & 2.503924591 \\ \mathrm{C} & 9.261176171 & -0.323222540 & 1.975025996 \\ \mathrm{C} & 9.550822204 & -0.775780569 & 3.394131907 \\ \mathrm{C} & 5.471356995 & -0.167517984 & 0.929024020 \\ \mathrm{C} & 4.908404404 & 1.085010394 & 0.278801981 \\ \mathrm{~N} & 10.066319298 & 0.353635784 & 4.230219601 \\ \mathrm{~N} & 4.123375227 & 0.771912712 & -0.966036608 \\ \mathrm{H} & 10.168737718 & 0.042359534 & 1.462633910 \\ \mathrm{H} & 8.891738553 & -1.180612517 & 1.393489034 \\ \mathrm{H} & 10.299093074 & -1.582067042 & 3.412821723 \\ \mathrm{H} & 8.635518280 & -1.145689344 & 3.880820152 \\ \mathrm{H} & 6.163912356 & -0.712729006 & 0.267893404 \\ \mathrm{H} & 4.670511122 & -0.865630852 & 1.229861490 \\ \mathrm{H} & 4.227429457 & 1.615776504 & 0.959666311 \\ \mathrm{H} & 5.711759538 & 1.775799405 & -0.017016232 \\ \mathrm{H} & 11.009531554 & 0.654638940 & 3.946945708 \\ \mathrm{H} & 10.111369125 & 0.112279459 & 5.229665308 \\ \mathrm{H} & 9.439664847 & 1.171831835 & 4.122656496 \\ \mathrm{H} & 3.318164918 & 0.158168879 & -0.774245398 \\ \mathrm{H} & 4.693814581 & 0.302399520 & -1.683776872 \\ \mathrm{H} & 3.746368071 & 1.628267705 & -1.398424203 \\ & & & \\ \mathrm{~S} & 8.053680378 & 1.210525659 & 1.906677687 \\ \mathrm{~S} & 6.307701979 & 0.352993897 & 2.600099056 \\ \mathrm{C} & 9.218298505 & -0.195227528 & 1.843211265 \\ \mathrm{C} & 9.468079124 & -0.847578476 & 3.182744106 \\ \mathrm{C} & 5.477676559 & -0.187143938 & 1.076268024 \\ \mathrm{C} & 5.003160583 & 1.000154859 & 0.264771237 \\ \mathrm{~N} & 9.958386331 & 0.130417826 & 4.191549794\end{array}$




\begin{tabular}{|c|c|c|c|}
\hline $\mathrm{N}$ & 4.301199508 & 0.577350292 & -0.976543214 \\
\hline $\mathrm{H}$ & 10.139628512 & 0.241247180 & 1.419230946 \\
\hline $\mathrm{H}$ & 8.864973046 & -0.952057505 & 1.128183878 \\
\hline $\mathrm{H}$ & 10.219312009 & -1.644384965 & 3.094698800 \\
\hline $\mathrm{H}$ & 8.548141479 & -1.293461411 & 3.587070130 \\
\hline $\mathrm{H}$ & 6.142789459 & -0.853415048 & 0.505278422 \\
\hline $\mathrm{H}$ & 4.633690304 & -0.796165317 & 1.442577229 \\
\hline $\mathrm{H}$ & 4.311090394 & 1.620048469 & 0.848757574 \\
\hline $\mathrm{H}$ & 5.853683999 & 1.625247249 & -0.039653407 \\
\hline $\mathrm{H}$ & 10.866497088 & 0.541838931 & 3.943505571 \\
\hline $\mathrm{H}$ & 10.075570932 & -0.303781763 & 5.115888729 \\
\hline $\mathrm{H}$ & 9.291556108 & 0.907402858 & 4.286793652 \\
\hline $\mathrm{H}$ & 3.486651480 & -0.026232968 & -0.789510735 \\
\hline $\mathrm{H}$ & 4.913538133 & 0.061463276 & -1.625471695 \\
\hline $\mathrm{H}$ & 3.940322665 & 1.389475691 & -1.502021698 \\
\hline \multicolumn{4}{|c|}{$\mathrm{H}_{4} \mathrm{HCysS}^{2+}$} \\
\hline $\mathrm{S}$ & 5.479652940 & 3.219981089 & 0.593647602 \\
\hline $\mathrm{S}$ & 6.195095437 & 3.459869309 & 2.515724631 \\
\hline $\mathrm{O}$ & 0.087502160 & 3.217118564 & 2.130694666 \\
\hline $\mathrm{O}$ & 1.677356440 & 3.041436566 & 3.710908983 \\
\hline $\mathrm{O}$ & 12.223711610 & 3.800298889 & 0.792630982 \\
\hline $\mathrm{O}$ & 10.619057639 & 3.366016911 & -0.722001985 \\
\hline $\mathrm{N}$ & 1.605211044 & 2.876697538 & 0.141442121 \\
\hline $\mathrm{N}$ & 10.973338380 & 2.956062713 & 2.810704660 \\
\hline $\mathrm{C}$ & 1.231615685 & 3.075977710 & 2.483943389 \\
\hline $\mathrm{C}$ & 2.345387306 & 2.943361550 & 1.441835378 \\
\hline $\mathrm{C}$ & 3.296862986 & 1.779593778 & 1.675413615 \\
\hline $\mathrm{C}$ & 4.473567134 & 1.698164147 & 0.709588216 \\
\hline $\mathrm{C}$ & 11.139394001 & 3.381055776 & 0.474402674 \\
\hline $\mathrm{C}$ & 10.213080167 & 2.764116577 & 1.532419587 \\
\hline $\mathrm{C}$ & 8.814054521 & 3.369926840 & 1.552581562 \\
\hline $\mathrm{C}$ & 7.826071976 & 2.643083573 & 2.455949625 \\
\hline $\mathrm{H}$ & 2.920666043 & 3.885388910 & 1.443285297 \\
\hline $\mathrm{H}$ & 3.679561345 & 1.896083964 & 2.701823152 \\
\hline $\mathrm{H}$ & 2.742930188 & 0.824162340 & 1.664724235 \\
\hline $\mathrm{H}$ & 5.113058134 & 0.844551039 & 0.978902552 \\
\hline $\mathrm{H}$ & 4.158201440 & 1.523463304 & -0.335088421 \\
\hline $\mathrm{H}$ & 10.161901196 & 1.681429039 & 1.330570282 \\
\hline $\mathrm{H}$ & 8.871314718 & 4.438640103 & 1.824204223 \\
\hline $\mathrm{H}$ & 8.443289008 & 3.337110515 & 0.515867122 \\
\hline $\mathrm{H}$ & 7.710491821 & 1.586875940 & 2.163925891 \\
\hline $\mathrm{H}$ & 8.133106043 & 2.653248116 & 3.517310875 \\
\hline $\mathrm{H}$ & 0.618132404 & 3.139976778 & 0.403720011 \\
\hline $\mathrm{H}$ & 1.963926589 & 3.515049923 & -0.576805924 \\
\hline $\mathrm{H}$ & 1.573663683 & 1.928696558 & -0.253292319 \\
\hline $\mathrm{H}$ & 11.117814331 & 2.097341502 & 3.352574162 \\
\hline $\mathrm{H}$ & 11.905484111 & 3.333485534 & 2.484955275 \\
\hline $\mathrm{H}$ & 10.549240951 & 3.659848436 & 3.427243417 \\
\hline $\mathrm{H}$ & 11.244481730 & 3.742455373 & -1.368212774 \\
\hline $\mathrm{H}$ & 0.941114245 & 3.157208108 & 4.339027268 \\
\hline
\end{tabular}




\begin{tabular}{|c|c|c|c|}
\hline \multicolumn{4}{|c|}{$\mathrm{H}_{4} \mathrm{HCysS}^{2+} \mathrm{SMD}$} \\
\hline S & 5.516841811 & 3.675022292 & 0.612164852 \\
\hline S & .161637630 & 3.742203729 & 2.569370709 \\
\hline $\mathrm{O}$ & 0.173380406 & 2.657105542 & 2.308834020 \\
\hline $\mathrm{O}$ & 1.951989895 & 2.878193896 & 3.671634959 \\
\hline & 12.132578454 & 3.515928348 & 0.659114638 \\
\hline & 10.414352010 & 2.972652714 & -0.685518009 \\
\hline $\mathrm{N}$ & 1.591330306 & 2.740711332 & 0.093901620 \\
\hline & 10.836363816 & 3.031502362 & 2.886699246 \\
\hline $\mathrm{C}$ & 1.356570598 & 2.797474524 & 6096966 \\
\hline $\mathrm{C}$ & 2.357808007 & 2.909964905 & 59989068 \\
\hline $\mathrm{C}$ & 3.499563544 & 1.909113611 & 2215 \\
\hline $\mathrm{C}$ & 4.645928439 & 2.074285815 & 0169 \\
\hline $\mathrm{C}$ & 11.00 & 3.112739406 & 0129 \\
\hline y & 10.108192904 & 2.681800817 & 301587 \\
\hline $\mathrm{C}$ & 8.733568731 & 3.336021090 & 38227 \\
\hline $\mathrm{C}$ & 7.715 & 2.788547634 & \\
\hline $\mathrm{H}$ & 2.7 & 874725 & \\
\hline $\mathrm{H}$ & 3.8 & 2.029017947 & 0546 \\
\hline $\mathrm{H}$ & 3.11 & & 1067 \\
\hline $\mathrm{H}$ & & & \\
\hline $\mathrm{H}$ & 4.3 & & \\
\hline & 10.0 & 83719420 & 9435 \\
\hline $\mathrm{H}$ & 8.8 & & \\
\hline $\mathrm{H}$ & 8.3 & 395 & \\
\hline $\mathrm{H}$ & & 1.727697607 & \\
\hline $\mathrm{H}$ & 8.0 & 2.864072116 & \\
\hline $\mathrm{H}$ & 0.8 & & \\
\hline $\mathrm{H}$ & 2.1 & 5889991 & \\
\hline $\mathrm{H}$ & & 1.825850562 & 2337 \\
\hline & 6919 & 2.697588453 & 0086 \\
\hline $\mathrm{H}$ & 94 & 86723 & \\
\hline $\mathrm{H}$ & 513 & 82316 & 0953 \\
\hline & 11.002 & 3.247756646 & 2531 \\
\hline $\mathrm{H}$ & 1.312966614 & 2.800346446 & 4.405782934 \\
\hline & & & \\
\hline 3 & & & \\
\hline $\mathrm{S}$ & -0.2 & -1 . & \\
\hline $\mathrm{C}$ & 7461 & 0111256 & -1.1 \\
\hline $\mathrm{C}$ & -1.450881805 & -1.130119099 & 5201 \\
\hline $\mathrm{C}$ & 1398 & 0.387363976 & -0.8 \\
\hline $\mathrm{C}$ & -2.73 & -0.387362357 & \\
\hline $\mathrm{C}$ & 3.572819136 & 1.062640370 & 0.190779167 \\
\hline $\mathrm{C}$ & 821782 & -1.062631186 & 779821 \\
\hline $\mathrm{O}$ & 636416 & & \\
\hline $\mathrm{O}$ & -4.472631196 & -0.228148117 & 0.721937829 \\
\hline $\mathrm{O}$ & 3.482191606 & 2.218736153 & 0.509119221 \\
\hline $\mathrm{O}$ & -3.482202884 & -2.218733526 & 0.50912157 \\
\hline $\mathrm{H}$ & & 2.212168660 & \\
\hline 11 & -1.643715525 & -2.212175548 & -1.22587433 \\
\hline $\mathrm{H}$ & 0.986109123 & 0.794622970 & -2.08489095 \\
\hline
\end{tabular}




$\begin{array}{rrrr}\mathrm{H} & -0.986117538 & -0.794639687 & -2.084898767 \\ \mathrm{H} & 2.546647855 & -0.658329625 & -0.581244719 \\ \mathrm{H} & -2.546640004 & 0.658330931 & -0.581249159 \\ \mathrm{H} & 3.360115789 & 0.344662122 & -1.781140148 \\ \mathrm{H} & -3.360115298 & -0.344658575 & -1.781140792 \\ \mathrm{H} & 4.983666939 & 0.743345584 & 1.365430330 \\ \mathrm{H} & -4.983666699 & -0.743318068 & 1.365430443\end{array}$

\begin{tabular}{|c|c|c|c|}
\hline \multicolumn{4}{|c|}{$\mathrm{H}_{2}$ DTDPA SMD } \\
\hline $\mathrm{S}$ & 0.196632509 & 0.977653144 & 0.187717305 \\
\hline S & -0.207159629 & -1.044050344 & 0.172828147 \\
\hline $\mathrm{C}$ & 1.420712809 & 1.167325874 & -1.152565559 \\
\hline $\mathrm{C}$ & -1.432988456 & -1.217186988 & -1.168976750 \\
\hline $\mathrm{C}$ & 2.726026072 & 0.451142749 & -0.898864547 \\
\hline $\mathrm{C}$ & -2.719908241 & -0.462764463 & -0.930423604 \\
\hline $\mathrm{C}$ & 3.528678975 & 1.057122798 & 0.216541182 \\
\hline $\mathrm{C}$ & -3.525448413 & -1.011399859 & 0.213473598 \\
\hline $\mathrm{O}$ & 4.512322187 & 0.243050395 & 0.620365962 \\
\hline $\mathrm{O}$ & -4.422341972 & -0.125161029 & 0.659813692 \\
\hline $\mathrm{O}$ & 3.349388325 & 2.154134708 & 0.691563596 \\
\hline $\mathrm{O}$ & -3.419722703 & -2.126345091 & 0.669325850 \\
\hline $\mathrm{H}$ & 1.573067051 & 2.256844397 & -1.214419864 \\
\hline $\mathrm{H}$ & -1.615597939 & -2.302930000 & 03706 \\
\hline $\mathrm{H}$ & 0.966415543 & 0.843876226 & 073587 \\
\hline $\mathrm{H}$ & -0.970578631 & -0.922633963 & -2.122004328 \\
\hline $\mathrm{H}$ & 2.562233030 & -0.618021146 & -0.690341102 \\
\hline $\mathrm{H}$ & -2.532417908 & 0.609628243 & 29741 \\
\hline $\mathrm{H}$ & 3.360755372 & 49206 & 6847 \\
\hline $\mathrm{H}$ & -3.363401576 & -0.521894562 & -1.826849945 \\
\hline $\mathrm{H}$ & 5.033851491 & 0.681667328 & 1.316903499 \\
\hline $\mathrm{H}$ & -4.957305159 & -0.526592440 & 1.368806479 \\
\hline & & $\mathrm{H}_{3} \mathrm{Cys}^{+}$ & \\
\hline $\mathrm{S}$ & 0.571029987 & -0.0 & \\
\hline $\mathrm{O}$ & 4.390114646 & 387743 & 22401 \\
\hline $\mathrm{O}$ & 3.864080578 & -1.535508330 & 1.496038442 \\
\hline $\mathrm{N}$ & 3.140581878 & 1.211154437 & -0.659216252 \\
\hline $\mathrm{C}$ & 757617 & 758442 & 03651 \\
\hline $\mathrm{C}$ & 3.275312503 & 0.606759984 & 0.691498653 \\
\hline $\mathrm{C}$ & 1.928640335 & 0.567993944 & 1.417917789 \\
\hline $\mathrm{H}$ & 2.177629139 & 0.965584747 & -0.993201220 \\
\hline $\mathrm{H}$ & 3.822454499 & 0.729879757 & 755813 \\
\hline $\mathrm{H}$ & 3.267932701 & 2.227618960 & -0.686736104 \\
\hline $\mathrm{H}$ & 4.003049778 & 1.193485381 & 1.276901128 \\
\hline $\mathrm{H}$ & 4.318705833 & -2.377447581 & 95806 \\
\hline $\mathrm{H}$ & 1.635880609 & 1.581361813 & 1.733026582 \\
\hline $\mathrm{H}$ & 2.029239258 & -0.036857814 & 2.329541700 \\
\hline $\mathrm{H}$ & 0.933212912 & -1.330756042 & 0.346365904 \\
\hline & & ${ }_{3} \mathrm{Cys}^{+} \mathrm{SMD}$ & \\
\hline $\mathrm{S}$ & 0.548517367 & -0.092719972 & 0.419207382 \\
\hline $\mathrm{O}$ & 4.327205190 & -1.093835752 & -0.642402982 \\
\hline
\end{tabular}




$\begin{array}{rrrr}\mathrm{O} & 3.892558817 & -1.508159026 & 1.523841922 \\ \mathrm{~N} & 3.148878228 & 1.237334259 & -0.659428457 \\ \mathrm{C} & 3.886217783 & -0.769159600 & 0.433744015 \\ \mathrm{C} & 3.264584046 & 0.602274036 & 0.677366892 \\ \mathrm{C} & 1.926615180 & 0.548856689 & 1.412741199 \\ \mathrm{H} & 2.186559809 & 1.102521274 & -1.025465929 \\ \mathrm{H} & 3.773449923 & 0.741182215 & -1.318361640 \\ \mathrm{H} & 3.360738486 & 2.244994977 & -0.683357851 \\ \mathrm{H} & 3.975781215 & 1.178912492 & 1.289297905 \\ \mathrm{H} & 4.321167770 & -2.372281206 & 1.367869204 \\ \mathrm{H} & 1.628120057 & 1.564308338 & 1.710295799 \\ \mathrm{H} & 2.047916531 & -0.034646812 & 2.334433494 \\ \mathrm{H} & 0.977311872 & -1.371304993 & 0.316874242\end{array}$

\begin{tabular}{lrrr} 
& \multicolumn{3}{c}{$\mathrm{H}_{2} \mathrm{CysO}_{2} \mathrm{H}^{+}$} \\
$\mathrm{S}$ & 0.472622895 & -0.167263475 & 0.821114284 \\
$\mathrm{O}$ & 4.254693375 & -1.332062714 & -0.374447810 \\
$\mathrm{O}$ & 4.008190681 & -1.374856949 & 1.862227488 \\
$\mathrm{~N}$ & 2.970686410 & 0.894349756 & -0.717331487 \\
$\mathrm{C}$ & 3.866096954 & -0.866302050 & 0.662322248 \\
$\mathrm{C}$ & 3.198970327 & 0.514711225 & 0.703882439 \\
$\mathrm{C}$ & 1.940989859 & 0.617155420 & 1.565256731 \\
$\mathrm{H}$ & 1.918877095 & 0.748077769 & -0.930127981 \\
$\mathrm{H}$ & 3.534023252 & 0.231619968 & -1.286647529 \\
$\mathrm{H}$ & 3.234415071 & 1.858433751 & -0.936178410 \\
$\mathrm{H}$ & 3.954738829 & 1.188656440 & 1.141840011 \\
$\mathrm{H}$ & 4.468719154 & -2.229764047 & 1.803667022 \\
$\mathrm{H}$ & 1.640520988 & 1.665697368 & 1.716358515 \\
$\mathrm{H}$ & 2.124259854 & 0.160263568 & 2.548845121 \\
$\mathrm{O}$ & 1.223092893 & -1.633259465 & 0.714562815 \\
$\mathrm{O}$ & 0.444291614 & 0.446312394 & -0.551691322 \\
$\mathrm{H}$ & 0.572519279 & -2.320406449 & 0.491906810
\end{tabular}

\begin{tabular}{lrrr} 
& \multicolumn{3}{c}{$\mathrm{H}_{2} \mathrm{CysO}_{2} \mathrm{H}^{+} \mathrm{SMD}$} \\
$\mathrm{S}$ & 0.227485410 & -0.045383316 & 0.880031969 \\
$\mathrm{O}$ & 5.152105914 & 0.033726243 & 0.264451284 \\
$\mathrm{O}$ & 4.481427128 & -1.606273442 & 1.658876134 \\
$\mathrm{~N}$ & 2.786252488 & 0.115173345 & -1.015702579 \\
$\mathrm{C}$ & 4.312682452 & -0.706897330 & 0.709393769 \\
$\mathrm{C}$ & 2.867413289 & -0.678897079 & 0.238429221 \\
$\mathrm{C}$ & 1.980021016 & -0.104838014 & 1.335742855 \\
$\mathrm{H}$ & 1.779694734 & 0.405245888 & -1.124657896 \\
$\mathrm{H}$ & 3.067275918 & -0.436542538 & -1.838568700 \\
$\mathrm{H}$ & 3.395685054 & 0.945603735 & -0.981037408 \\
$\mathrm{H}$ & 2.546703715 & -1.703628686 & 0.001252554 \\
$\mathrm{H}$ & 5.387651088 & -1.588243586 & 2.023417859 \\
$\mathrm{H}$ & 2.250439342 & 0.930168479 & 1.596173244 \\
$\mathrm{H}$ & 2.054536706 & -0.716992745 & 2.245485641 \\
$\mathrm{O}$ & 0.120661740 & -1.670601673 & 0.657645808 \\
$\mathrm{O}$ & 0.226516729 & 0.612811356 & -0.474962519 \\
$\mathrm{H}$ & -0.815778006 & -1.941081537 & 0.703433361
\end{tabular}




$\begin{array}{crrr} & & \mathrm{H}_{2} \text { Cyste }^{+} & \\ \mathrm{S} & 7.676308109 & 0.087104794 & 3.431770076 \\ \mathrm{C} & 7.571237550 & -1.436085011 & 2.416494474 \\ \mathrm{C} & 8.837072381 & -2.265621036 & 2.584441685 \\ \mathrm{~N} & 10.035447356 & -1.413323861 & 2.322671113 \\ \mathrm{H} & 7.398761761 & -1.171591230 & 1.362660405 \\ \mathrm{H} & 6.712376979 & -2.040322180 & 2.742904153 \\ \mathrm{H} & 8.855688426 & -3.130132839 & 1.905889190 \\ \mathrm{H} & 8.932728197 & -2.633179584 & 3.616610543 \\ \mathrm{H} & 10.181915365 & -1.229520221 & 1.322472460 \\ \mathrm{H} & 10.905322007 & -1.802049326 & 2.704427382 \\ \mathrm{H} & 9.826826482 & -0.494887338 & 2.789093561 \\ \mathrm{H} & 7.428743334 & 0.973621704 & 2.443830197\end{array}$

\begin{tabular}{|c|c|c|c|}
\hline \multicolumn{4}{|c|}{$\mathrm{H}_{2}$ Cyste $^{+}$SMD } \\
\hline $\mathrm{S}$ & 7.497636076 & 0.048040053 & 3.500439983 \\
\hline $\mathrm{C}$ & 7.555918365 & -1.428834868 & 2.419090061 \\
\hline $\mathrm{C}$ & 8.834439739 & -2.220913047 & 2.595569099 \\
\hline $\mathrm{N}$ & 10.056895274 & -1.420582915 & 2.290778812 \\
\hline $\mathrm{H}$ & 7.401287490 & -1.116867852 & 1.376293108 \\
\hline $\mathrm{H}$ & 6.717966911 & -2.091621160 & 2.681638194 \\
\hline $\mathrm{H}$ & 8.834004555 & -3.106918808 & 1.945637888 \\
\hline $\mathrm{H}$ & 8.933991187 & -2.567256883 & 3.63312268 \\
\hline $\mathrm{H}$ & 10.247087483 & -1.337828534 & 1.28232063 \\
\hline $\mathrm{H}$ & 10.891716969 & -1.815101530 & 2.736076156 \\
\hline $\mathrm{H}$ & 9.948264282 & -0.461784341 & 2.66 \\
\hline $\mathrm{H}$ & 7.443219616 & 0.963683756 & 2.515948010 \\
\hline \multicolumn{4}{|c|}{$\mathrm{HCysteO}_{2} \mathrm{H}^{+}$} \\
\hline $\mathrm{S}$ & 7.203339500 & 0.034695914 & 3.246494769 \\
\hline $\mathrm{C}$ & 7.469803486 & -1.501773642 & 2.275852839 \\
\hline $\mathrm{C}$ & 8.745582633 & -2.205757974 & 2.701281831 \\
\hline $\mathrm{N}$ & 9.926118582 & -1.348684872 & 2.39 \\
\hline $\mathrm{H}$ & 7.489659898 & -1.185571205 & 1.21 \\
\hline $\mathrm{H}$ & 6.593644940 & -2.150556945 & 2.422461403 \\
\hline $\mathrm{H}$ & 8.842257083 & -3.173570257 & 2.188331696 \\
\hline $\mathrm{H}$ & 8.761441430 & -2.400387326 & 3.784430568 \\
\hline $\mathrm{H}$ & 10.092458854 & -1.255325406 & 1.385192035 \\
\hline $\mathrm{H}$ & 10.789097449 & -1.692685853 & 2.826623981 \\
\hline $\mathrm{H}$ & 9.653479793 & -0.377559435 & 2.803187570 \\
\hline $\mathrm{O}$ & 8.611295967 & 0.530731897 & 3.459952038 \\
\hline $\mathrm{O}$ & 6.543114484 & 0.802248296 & 1.960507221 \\
\hline $\mathrm{H}$ & 6.024080965 & 1.564061880 & 2.270726948 \\
\hline \multicolumn{4}{|c|}{$\mathrm{HCysteO}_{2} \mathrm{H}^{+} \mathrm{SMD}$} \\
\hline $\mathrm{S}$ & 7.224611671 & 0.061933301 & 3.226077970 \\
\hline $\mathrm{C}$ & 7.520155347 & -1.420767183 & 2.222316202 \\
\hline $\mathrm{C}$ & 8.734651782 & -2.171559905 & 2.724810448 \\
\hline $\mathrm{N}$ & 9.988619454 & -1.438409804 & 2.404111528 \\
\hline $\mathrm{H}$ & 7.631779116 & -1.070775711 & 1.182396101 \\
\hline $\mathrm{H}$ & 6.616683969 & -2.046140678 & 2.282260120 \\
\hline $\mathrm{H}$ & 8.778326737 & -3.166397351 & 2.26277420 \\
\hline
\end{tabular}




$\begin{array}{rrrr}\mathrm{H} & 8.707074116 & -2.322880562 & 3.813890488 \\ \mathrm{H} & 10.203096198 & -1.432066439 & 1.395803500 \\ \mathrm{H} & 10.796945041 & -1.836267204 & 2.890448497 \\ \mathrm{H} & 9.860823147 & -0.448333786 & 2.712662571 \\ \mathrm{O} & 8.587712569 & 0.696561247 & 3.312778620 \\ \mathrm{O} & 6.358495189 & 0.807541879 & 2.043672308 \\ \mathrm{H} & 5.736400731 & 1.427427266 & 2.464031667\end{array}$

$\mathrm{H}_{2} \mathrm{MPA}$

$\begin{array}{llll}\mathrm{S} & -1.870576290 & 0.593962405 & 0.283160267 \\ \mathrm{C} & -1.601063159 & 1.793773992 & 1.620269832 \\ \mathrm{C} & -1.179263851 & 3.166897005 & 1.133981511 \\ \mathrm{C} & -1.079073837 & 4.166232435 & 2.256133861 \\ \mathrm{O} & -1.373092433 & 3.954433379 & 3.403205515 \\ \mathrm{O} & -0.622675506 & 5.350159504 & 1.828186846 \\ \mathrm{H} & -0.881384776 & 1.396429996 & 2.350842574 \\ \mathrm{H} & -2.563584200 & 1.860755840 & 2.148946492 \\ \mathrm{H} & -0.206789139 & 3.135091073 & 0.615363387 \\ \mathrm{H} & -1.892126990 & 3.558715430 & 0.389421216 \\ \mathrm{H} & -0.599473251 & 5.935110218 & 2.601304091 \\ \mathrm{H} & -0.585847341 & 0.527470158 & -0.130104598\end{array}$

\begin{tabular}{lrrr}
\multicolumn{4}{c}{$\mathrm{H}_{2}$ MPA SMD } \\
S & -1.825169303 & 0.579134782 & 0.281130411 \\
$\mathrm{C}$ & -1.626723180 & 1.791430867 & 1.621861536 \\
$\mathrm{C}$ & -1.205261255 & 3.164275436 & 1.136366161 \\
$\mathrm{C}$ & -1.068726124 & 4.154948644 & 2.257576998 \\
$\mathrm{O}$ & -1.325594610 & 3.932434237 & 3.418502340 \\
$\mathrm{O}$ & -0.626008138 & 5.341165616 & 1.824467445 \\
$\mathrm{H}$ & -0.918966114 & 1.406082019 & 2.370072740 \\
$\mathrm{H}$ & -2.610231121 & 1.841017964 & 2.112861395 \\
$\mathrm{H}$ & -0.238635893 & 3.124494761 & 0.606550706 \\
$\mathrm{H}$ & -1.926181513 & 3.573690725 & 0.409369530 \\
$\mathrm{H}$ & -0.551915894 & 5.959080886 & 2.574342032 \\
$\mathrm{H}$ & -0.531537634 & 0.571275513 & -0.112390291 \\
& & & \\
& & $\mathrm{H}_{2} \mathrm{SPA}$ & \\
$\mathrm{S}$ & -1.635842616 & 0.774921092 & 0.197838696 \\
$\mathrm{C}$ & -1.480131240 & 1.880660296 & 1.629660278 \\
$\mathrm{C}$ & -1.072681719 & 3.261973981 & 1.171756650 \\
$\mathrm{C}$ & -1.129348514 & 4.265231936 & 2.292853540 \\
$\mathrm{O}$ & -1.556678399 & 4.051962481 & 3.396058326 \\
$\mathrm{O}$ & -0.645811003 & 5.452771331 & 1.911864157 \\
$\mathrm{H}$ & -0.765580473 & 1.423707645 & 2.329920729 \\
$\mathrm{H}$ & -2.474936520 & 1.885880323 & 2.099213736 \\
$\mathrm{H}$ & -0.059088718 & 3.285055466 & 0.738658987 \\
$\mathrm{H}$ & -1.751334762 & 3.603929405 & 0.371315776 \\
$\mathrm{H}$ & -0.730084240 & 6.046657057 & 2.673790235 \\
$\mathrm{O}$ & -2.441295533 & -0.358881963 & 1.132402534 \\
$\mathrm{H}$ & -2.474122171 & -1.175974937 & 0.614977032 \\
$\mathrm{O}$ & -2.582544283 & 1.451963813 & -0.719634605
\end{tabular}




\begin{tabular}{lrrr} 
& \multicolumn{3}{c}{$\mathrm{H}_{2} \mathrm{SPA}$ SMD } \\
$\mathrm{S}$ & -1.597392481 & 0.753865696 & 0.231104374 \\
$\mathrm{C}$ & -1.527467591 & 1.884496401 & 1.645438475 \\
$\mathrm{C}$ & -1.105325885 & 3.261476014 & 1.184516224 \\
$\mathrm{C}$ & -1.081595219 & 4.254024548 & 2.311562321 \\
$\mathrm{O}$ & -1.326041198 & 4.001662081 & 3.467795109 \\
$\mathrm{O}$ & -0.749067361 & 5.473448789 & 1.878452086 \\
$\mathrm{H}$ & -0.832238562 & 1.446121762 & 2.377435290 \\
$\mathrm{H}$ & -2.542363258 & 1.883733071 & 2.071079997 \\
$\mathrm{H}$ & -0.102598705 & 3.260254921 & 0.723465071 \\
$\mathrm{H}$ & -1.792679738 & 3.640021757 & 0.411249466 \\
$\mathrm{H}$ & -0.729733894 & 6.100501510 & 2.624224436 \\
$\mathrm{O}$ & -2.518970526 & -0.348121218 & 1.069356582 \\
$\mathrm{H}$ & -2.438187277 & -1.207033655 & 0.619589160 \\
$\mathrm{O}$ & -2.455818505 & 1.445406266 & -0.774592510
\end{tabular}

$\begin{array}{lr}\mathrm{S} & 0.018360909 \\ \mathrm{O} & 1.627853728 \\ \mathrm{O} & -0.505260900 \\ \mathrm{~N} & 1.633284491 \\ \mathrm{C} & 0.586600690 \\ \mathrm{C} & 0.425538077 \\ \mathrm{C} & -0.873887456 \\ \mathrm{C} & -0.914128774 \\ \mathrm{H} & 0.505950076 \\ \mathrm{H} & -1.099176779 \\ \mathrm{H} & -1.668066493 \\ \mathrm{H} & -1.958387067 \\ \mathrm{H} & -0.475832007 \\ \mathrm{H} & 1.558734239 \\ \mathrm{H} & 1.734298981 \\ \mathrm{H} & 2.455144348 \\ \mathrm{H} & -0.353819689 \\ \mathrm{H} & -0.612081791\end{array}$

$\mathrm{H}_{3} \mathrm{HCys}^{+} \mathrm{SMD}$

$\mathrm{S}$

$\mathrm{O}$

$\mathrm{O}$

$\mathrm{N}$

C

C

C

C

$\mathrm{H}$

$\mathrm{H}$

$\mathrm{H}$

$\mathrm{H}$

$\mathrm{H}$

$\mathrm{H}$

$\mathrm{H}$
$\mathrm{H}_{3} \mathrm{HCys}^{+}$

$\begin{array}{ll}0.318348269 & -0.030748546\end{array}$

$\begin{array}{ll}5.465368401 & -0.406426132\end{array}$

$5.421376747 \quad-1.120954653$

$3.006976726 \quad 0.453963068$

$\begin{array}{ll}4.899714988 & -0.611396345\end{array}$

$3.414169180 \quad-0.316554742$

$3.012973900 \quad 0.370783380$

$\begin{array}{ll}1.576683030 & 0.906155636\end{array}$

$\begin{array}{ll}2.877764025 & -1.277428978\end{array}$

$3.706926588 \quad 1.198684069$

$3.172238120 \quad-0.373771160$

$\begin{array}{ll}1.252902217 & 1.020716120\end{array}$

$\begin{array}{ll}1.513544760 & 1.915677357\end{array}$

$\begin{array}{ll}3.247373199 & 1.449809779\end{array}$

$\begin{array}{ll}1.975693433 & 0.354150364\end{array}$

$\begin{array}{ll}3.517956841 & 0.091585318\end{array}$

$6.360156268 \quad-1.329145954$

$\begin{array}{ll}0.454411487 & -1.219766549\end{array}$

\begin{tabular}{lrrr} 
& \multicolumn{3}{c}{$\mathrm{H}_{3} \mathrm{HCys}^{+} \mathrm{SMD}$} \\
$\mathrm{S}$ & -0.153173526 & 0.310958373 & -0.224398340 \\
$\mathrm{O}$ & 1.427852375 & 5.602279898 & -0.162576456 \\
$\mathrm{O}$ & -0.464694446 & 5.274252040 & -1.335059539 \\
$\mathrm{~N}$ & 1.678236315 & 3.043504403 & 0.509712303 \\
$\mathrm{C}$ & 0.543077085 & 4.891186695 & -0.569992283 \\
$\mathrm{C}$ & 0.470486309 & 3.410074199 & -0.269248668 \\
$\mathrm{C}$ & -0.832435845 & 2.999403660 & 0.406911658 \\
$\mathrm{C}$ & -0.899549013 & 1.550791280 & 0.885835656 \\
$\mathrm{H}$ & 0.565230872 & 2.875304067 & -1.229184176 \\
$\mathrm{H}$ & -1.049582402 & 3.660123834 & 1.262765594 \\
$\mathrm{H}$ & -1.620612090 & 3.186590674 & -0.337454309 \\
$\mathrm{H}$ & -1.948232405 & 1.287453221 & 1.086585403 \\
$\mathrm{H}$ & -0.366413686 & 1.415987109 & 1.840640558 \\
$\mathrm{H}$ & 1.724255620 & 3.452862812 & 1.447679626 \\
$\mathrm{H}$ & 1.715319229 & 2.019597220 & 0.611396690
\end{tabular}




$\begin{array}{rrrr}\mathrm{H} & 2.544697280 & 3.328483513 & 0.032414306 \\ \mathrm{H} & -0.417829974 & 6.228200501 & -1.537857070 \\ \mathrm{H} & -0.831507111 & 0.657524680 & -1.342838921 \\ & & & \\ \mathrm{~S} & -0.528954680 & 0.570360500 & -0.138474071 \\ \mathrm{O} & 1.108241465 & 3.978081202 & -1.510714989 \\ \mathrm{O} & -0.989410393 & 4.653083607 & -1.072979158 \\ \mathrm{~N} & 1.550664169 & 3.032768868 & 0.864596546 \\ \mathrm{C} & 0.170585835 & 4.108908916 & -0.772218863 \\ \mathrm{C} & 0.259907641 & 3.742797146 & 0.711535551 \\ \mathrm{C} & -0.961295151 & 3.014628989 & 1.299809517 \\ \mathrm{C} & -0.997115674 & 1.485561302 & 1.354471680 \\ \mathrm{H} & 0.338985237 & 4.713981450 & 1.228414729 \\ \mathrm{H} & -1.108160252 & 3.348290393 & 2.338887012 \\ \mathrm{H} & -1.832790675 & 3.380264289 & 0.740146158 \\ \mathrm{H} & -2.015102409 & 1.157859219 & 1.617030302 \\ \mathrm{H} & -0.320156822 & 1.061324566 & 2.114016307 \\ \mathrm{H} & 1.873781305 & 2.975129058 & 1.833084444 \\ \mathrm{H} & 1.435940888 & 2.029050865 & 0.417026824 \\ \mathrm{H} & 2.259621035 & 3.505802131 & 0.286452976 \\ \mathrm{H} & -0.980642616 & 4.925297760 & -2.005951000 \\ \mathrm{O} & -1.294646688 & 1.623095724 & -1.152259174 \\ \mathrm{O} & 0.957609411 & 0.806571013 & -0.236186168 \\ \mathrm{H} & -1.391537514 & 1.203484768 & -2.023494779\end{array}$

$\mathrm{H}_{3} \mathrm{HCSA}^{+} \mathrm{SMD}$

$\begin{array}{lrrr}\mathrm{S} & -0.518288088 & 0.610600075 & -0.166720779 \\ \mathrm{O} & 1.086063098 & 3.806624620 & -1.518500179 \\ \mathrm{O} & -0.959704363 & 4.633428054 & -1.090456361 \\ \mathrm{~N} & 1.547136318 & 3.098545648 & 0.975919280 \\ \mathrm{C} & 0.170642312 & 4.031213043 & -0.766561940 \\ \mathrm{C} & 0.241302252 & 3.753524966 & 0.729309931 \\ \mathrm{C} & -0.957912470 & 3.010193505 & 1.327967110 \\ \mathrm{C} & -0.952922039 & 1.481395611 & 1.357613539 \\ \mathrm{H} & 0.285002388 & 4.758043865 & 1.178878899 \\ \mathrm{H} & -1.083550109 & 3.322864793 & 2.376164663 \\ \mathrm{H} & -1.848281264 & 3.366165198 & 0.793666928 \\ \mathrm{H} & -1.951733679 & 1.117703860 & 1.648501943 \\ \mathrm{H} & -0.241899809 & 1.062886252 & 2.088999610 \\ \mathrm{H} & 1.776352540 & 3.007864714 & 1.973512984 \\ \mathrm{H} & 1.523242736 & 2.144747656 & 0.498830522 \\ \mathrm{H} & 2.298763577 & 3.631969261 & 0.522646448 \\ \mathrm{H} & -0.986631493 & 4.840135267 & -2.043258612 \\ \mathrm{O} & -1.349328035 & 1.650424146 & -1.137731569 \\ \mathrm{O} & 0.958685874 & 0.874908404 & -0.336330628 \\ \mathrm{H} & -1.415040278 & 1.272216633 & -2.034929348 \\ & & & \\ & & \mathrm{H}_{2} \mathrm{O} & \\ \mathrm{O} & 3.449620998 & 0.290576634 & -0.955227067 \\ \mathrm{H} & 3.449620998 & 1.049913633 & -0.359184067 \\ \mathrm{H} & 3.449620998 & -0.468760365 & -0.359184067\end{array}$




\begin{tabular}{|c|c|c|c|}
\hline \multicolumn{4}{|c|}{$\mathrm{H}_{2} \mathrm{O}$} \\
\hline $\mathrm{O}$ & 3.449621000 & 0.290576634 & -0.958219821 \\
\hline $\mathrm{H}$ & 3.449621000 & 1.047183422 & -0.357687690 \\
\hline $\mathrm{H}$ & 3.449621000 & -0.466030154 & -0.357687690 \\
\hline \multicolumn{4}{|c|}{$\left[\mathrm{Pd}_{2} \mathrm{Cl}_{6}\left(\mathrm{~S}, \mathrm{~S}^{\prime}-\mathrm{H}_{4} \mathrm{CysS}\right)\right]^{0} \cdot 12 \mathrm{H}_{2} \mathrm{O}$} \\
\hline $\mathrm{C}$ & 3.488868788 & -0.162087093 & -1.595816485 \\
\hline $\mathrm{C}$ & -4.149672173 & 1.449379864 & -1.956535087 \\
\hline $\mathrm{C}$ & 2.649691938 & -1.324699272 & -2.119576308 \\
\hline $\mathrm{C}$ & -2.814199436 & 2.156628957 & -2.237639899 \\
\hline $\mathrm{C}$ & 1.297465143 & -0.966636971 & -2.684587763 \\
\hline $\mathrm{C}$ & -1.971389533 & 1.430971675 & -3.269374954 \\
\hline $\mathrm{H}$ & 1.662209581 & -2.475758817 & -0.672763943 \\
\hline $\mathrm{H}$ & -2.097825759 & 1.512420480 & -0.401280631 \\
\hline $\mathrm{H}$ & 2.403904914 & -3.332558088 & -1.836437836 \\
\hline $\mathrm{H}$ & -1.151037107 & 2.751294825 & -1.055832775 \\
\hline $\mathrm{H}$ & 3.350413228 & -2.590947186 & -0.533410688 \\
\hline $\mathrm{H}$ & -2.713233769 & 3.050277536 & -0.323508626 \\
\hline $\mathrm{H}$ & 3.199267990 & -1.666866450 & -3.016619768 \\
\hline $\mathrm{H}$ & -3.055067452 & 3.145275658 & -2.665658375 \\
\hline $\mathrm{H}$ & 1.407813962 & -0.367497920 & -3.598440916 \\
\hline $\mathrm{H}$ & -2.546276297 & 1.305718017 & -4.211010603 \\
\hline $\mathrm{H}$ & 0.779500711 & -1.899452980 & -2.947722324 \\
\hline $\mathrm{H}$ & -1.028275970 & 1.936558538 & -3.516513770 \\
\hline $\mathrm{H}$ & 3.608427192 & 1.739568045 & -1.557236116 \\
\hline $\mathrm{H}$ & -5.378940292 & 0.134692062 & -2.650538915 \\
\hline $\mathrm{N}$ & 2.548266794 & -2.484270567 & -1.217363996 \\
\hline $\mathrm{N}$ & -2.136046662 & 2.385809649 & -0.950526902 \\
\hline $\mathrm{O}$ & 4.519591760 & -0.321296633 & -0.971163521 \\
\hline $\mathrm{O}$ & -4.693915392 & 1.550457451 & -0.870999992 \\
\hline $\mathrm{O}$ & 3.038991773 & 0.993731313 & -1.997231323 \\
\hline $\mathrm{O}$ & -4.581836228 & 0.772067445 & -2.964286096 \\
\hline S & 0.160085268 & 0.035021551 & -1.654911402 \\
\hline S & -1.625355715 & -0.283475496 & -2.777891218 \\
\hline $\mathrm{O}$ & 4.471771281 & -2.482423458 & 0.694903685 \\
\hline $\mathrm{O}$ & -3.707230565 & 3.731231850 & 0.694160121 \\
\hline $\mathrm{H}$ & 3.949792888 & -2.142631238 & 1.434963024 \\
\hline $\mathrm{H}$ & -3.343204888 & 3.215153072 & 1.446149485 \\
\hline $\mathrm{H}$ & 4.802930531 & -1.672171045 & 0.264733633 \\
\hline $\mathrm{H}$ & -4.450480354 & 3.180372003 & 0.398542079 \\
\hline $\mathrm{O}$ & 4.343472640 & 2.652399328 & -0.640816637 \\
\hline $\mathrm{O}$ & -6.199692907 & -0.836168149 & -2.072092320 \\
\hline $\mathrm{H}$ & -5.533547735 & -1.574442662 & -1.966928453 \\
\hline $\mathrm{H}$ & 3.682311360 & 2.877862975 & 0.049232962 \\
\hline $\mathrm{H}$ & 4.933127154 & 2.002640112 & -0.233096781 \\
\hline $\mathrm{H}$ & -6.334284362 & -0.493495550 & -1.178533304 \\
\hline $\mathrm{Pd}$ & -0.201332539 & -0.448084929 & 0.617656385 \\
\hline $\mathrm{Cl}$ & -0.502070376 & -0.535499003 & 2.889985293 \\
\hline $\mathrm{Cl}$ & 2.079739174 & -0.179312540 & 1.008948105 \\
\hline $\mathrm{Cl}$ & -2.484060312 & -0.815048188 & 0.338940875 \\
\hline $\mathrm{O}$ & 0.006145124 & -3.298447722 & -0.518256361 \\
\hline
\end{tabular}




\begin{tabular}{|c|c|c|c|}
\hline $\mathrm{O}$ & 2.141410865 & 2.863597620 & 0.974087112 \\
\hline $\mathrm{H}$ & -0.584350147 & -3.341519222 & -1.293966652 \\
\hline $\mathrm{H}$ & 2.053772333 & 1.895696049 & 1.140502555 \\
\hline $\mathrm{H}$ & -0.586423485 & -3.179149189 & 0.234199718 \\
\hline $\mathrm{H}$ & 2.014750829 & 3.288346588 & 1.831073266 \\
\hline $\mathrm{O}$ & 1.812955294 & -4.227658231 & -3.120713041 \\
\hline $\mathrm{O}$ & 0.471056979 & 3.209989401 & -1.208081689 \\
\hline $\mathrm{H}$ & 0.855870789 & -4.374233303 & -3.168469687 \\
\hline $\mathrm{H}$ & 0.998990177 & 3.233308121 & -0.383122304 \\
\hline $\mathrm{H}$ & 2.041637440 & -3.782862059 & -3.969353458 \\
\hline $\mathrm{H}$ & 0.943830570 & 2.565692518 & -1.753191614 \\
\hline $\mathrm{O}$ & -2.498395241 & 1.776861252 & 2.038837019 \\
\hline $\mathrm{H}$ & -2.914054450 & 0.953749412 & 1.732328880 \\
\hline $\mathrm{H}$ & -1.806196473 & 1.432148302 & 2.626206341 \\
\hline $\mathrm{Pd}$ & -0.990618234 & -1.536859776 & -4.623003839 \\
\hline $\mathrm{Cl}$ & -1.580584058 & -3.436843727 & -3.339324132 \\
\hline $\mathrm{Cl}$ & -0.057681348 & 0.301720400 & -5.686432030 \\
\hline $\mathrm{Cl}$ & -0.440117352 & -2.862109425 & -6.415163304 \\
\hline $\mathrm{O}$ & -4.221351587 & -2.517692657 & -1.752531076 \\
\hline $\mathrm{H}$ & -3.679352765 & -2.931924093 & -2.443694627 \\
\hline $\mathrm{H}$ & -3.567102530 & -2.100094031 & -1.168975882 \\
\hline $\mathrm{O}$ & -3.148794110 & 0.761139959 & -6.018169295 \\
\hline $\mathrm{H}$ & -2.244122365 & 0.675420198 & -6.360571542 \\
\hline $\mathrm{H}$ & -3.505515057 & -0.131785183 & -6.081940923 \\
\hline $\mathrm{O}$ & 2.406421192 & -2.582260892 & -5.165726134 \\
\hline $\mathrm{H}$ & 3.076190555 & -2.762615147 & -5.834799142 \\
\hline $\mathrm{H}$ & 1.565335424 & -2.539052081 & -5.673828666 \\
\hline \multicolumn{4}{|c|}{$\left[\mathrm{Pd}_{2} \mathrm{Cl}_{6}\left(\mathrm{~S}, \mathrm{~S}^{\prime}-\mathrm{H}_{4} \mathrm{CysS}\right)\right]^{0 \cdot 12 \mathrm{H}_{2} \mathrm{O} \text { SMD }}$} \\
\hline $\mathrm{C}$ & 3.114553931 & -0.523302542 & -0.917838809 \\
\hline $\mathrm{C}$ & -3.721007845 & 1.224287854 & -1.264374866 \\
\hline $\mathrm{C}$ & 2.484335783 & -1.669901297 & -1.705814714 \\
\hline $\mathrm{C}$ & -2.728881801 & 2.085900414 & -2.040153387 \\
\hline $\mathrm{C}$ & 1.248635987 & -1.265950838 & -2.504984145 \\
\hline $\mathrm{C}$ & -2.151749115 & 1.283128332 & -3.204503709 \\
\hline $\mathrm{H}$ & 1.571117763 & -3.501432665 & -1.338120579 \\
\hline $\mathrm{H}$ & -1.372201262 & 1.851828724 & -0.483796643 \\
\hline $\mathrm{H}$ & 3.112177706 & -3.368114981 & -0.689151000 \\
\hline $\mathrm{H}$ & -0.831442498 & 2.951423125 & -1.671018131 \\
\hline $\mathrm{H}$ & 1.886822019 & -2.607390490 & 0.121070853 \\
\hline $\mathrm{H}$ & -2.022914088 & 3.348219535 & -0.473814564 \\
\hline $\mathrm{H}$ & 3.222904975 & -1.975753149 & -2.465689215 \\
\hline $\mathrm{H}$ & -3.258563567 & 2.947328832 & -2.477315958 \\
\hline $\mathrm{H}$ & 1.532002054 & -0.861100845 & -3.483725352 \\
\hline $\mathrm{H}$ & -2.933535554 & 1.088180922 & -3.958995922 \\
\hline $\mathrm{H}$ & 0.619963141 & -2.148039523 & -2.670642157 \\
\hline $\mathrm{H}$ & -1.320657914 & 1.791847959 & -3.712402880 \\
\hline $\mathrm{H}$ & 3.670341665 & 1.369538733 & -1.098471972 \\
\hline $\mathrm{H}$ & -5.309624420 & 0.118008773 & -1.477819716 \\
\hline $\mathrm{N}$ & 2.243078566 & -2.842646851 & -0.845108224 \\
\hline $\mathrm{N}$ & -1.687410419 & 2.583541543 & -1.135506035 \\
\hline $\mathrm{O}$ & 3.471210962 & -0.642324598 & 0.233168378 \\
\hline
\end{tabular}




\begin{tabular}{|c|c|c|c|}
\hline $\mathrm{O}$ & -3.486472835 & 0.866668081 & -0.120549751 \\
\hline $\mathrm{O}$ & 3.213157463 & 0.556140694 & -1.641188467 \\
\hline $\mathrm{O}$ & -4.740228114 & 0.880264348 & -1.975150044 \\
\hline S & 0.207053126 & 0.033325231 & -1.749841358 \\
\hline S & -1.649475301 & -0.405129494 & -2.741180545 \\
\hline $\mathrm{O}$ & 1.873548659 & -2.399598942 & 1.829207776 \\
\hline $\mathrm{O}$ & -2.199855334 & 4.246594539 & 0.886943152 \\
\hline $\mathrm{H}$ & 1.044450221 & -2.123421607 & 2.262712183 \\
\hline $\mathrm{H}$ & -2.310558545 & 3.496647333 & 1.524688252 \\
\hline $\mathrm{H}$ & 2.426710027 & -1.603300297 & 1.817700950 \\
\hline $\mathrm{H}$ & -3.012963297 & 4.764655428 & 0.963559385 \\
\hline $\mathrm{O}$ & 4.195565593 & 2.481683049 & -0.495867288 \\
\hline $\mathrm{O}$ & -5.913197124 & -0.986540806 & -0.910670168 \\
\hline $\mathrm{H}$ & -5.188066506 & -1.671175299 & -1.019129873 \\
\hline $\mathrm{H}$ & 3.453476611 & 3.128090807 & -0.362954071 \\
\hline $\mathrm{H}$ & 4.541335478 & 2.274912025 & 0.385025793 \\
\hline $\mathrm{H}$ & -6.002661604 & -0.856679143 & 0.044326387 \\
\hline $\mathrm{Pd}$ & -0.288301290 & -0.531346879 & 0.412107897 \\
\hline $\mathrm{Cl}$ & -0.878774894 & -1.008295852 & 2.606957342 \\
\hline $\mathrm{Cl}$ & 0.463345320 & 1.572415461 & 1.123598006 \\
\hline $\mathrm{Cl}$ & -0.954226634 & -2.638309429 & -0.264798730 \\
\hline $\mathrm{O}$ & 0.756616913 & -4.439744102 & -2.468560238 \\
\hline $\mathrm{O}$ & 2.002615829 & 3.939565129 & -0.242334863 \\
\hline $\mathrm{H}$ & 1.176436348 & -4.169664845 & -3.323505030 \\
\hline $\mathrm{H}$ & 1.457270869 & 3.325751475 & 0.287500608 \\
\hline $\mathrm{H}$ & -0.186027071 & -4.227970682 & -2.585685559 \\
\hline $\mathrm{H}$ & 1.994341932 & 4.783926767 & 0.234260769 \\
\hline $\mathrm{O}$ & 1.988943642 & -3.351500478 & -4.587276811 \\
\hline $\mathrm{O}$ & 0.420781492 & 3.534733910 & -2.448941615 \\
\hline $\mathrm{H}$ & 2.704779428 & -3.865744050 & -4.986193693 \\
\hline $\mathrm{H}$ & 1.020309069 & 3.862675950 & -1.747934121 \\
\hline $\mathrm{H}$ & 1.331505361 & -3.230208423 & -5.301310879 \\
\hline $\mathrm{H}$ & 0.992221059 & 2.922914223 & -2.977238449 \\
\hline $\mathrm{O}$ & -2.600474702 & 1.978147923 & 2.280354927 \\
\hline $\mathrm{H}$ & -2.999368294 & 1.496384642 & 1.532684045 \\
\hline $\mathrm{H}$ & -1.753820847 & 1.520721234 & 2.407157498 \\
\hline $\mathrm{Pd}$ & -1.067670011 & -1.560712962 & -4.671344604 \\
\hline $\mathrm{Cl}$ & -2.096044524 & -3.429346242 & -3.735120159 \\
\hline $\mathrm{Cl}$ & -0.061815901 & 0.322497883 & -5.612170199 \\
\hline $\mathrm{Cl}$ & -0.465719107 & -2.807382839 & -6.524119864 \\
\hline $\mathrm{O}$ & -3.949220203 & -2.659144600 & -1.232165028 \\
\hline $\mathrm{H}$ & -3.620925783 & -2.846581324 & -2.129519083 \\
\hline $\mathrm{H}$ & -3.126278054 & -2.482504065 & -0.740960537 \\
\hline $\mathrm{O}$ & 2.041821668 & 1.926859042 & -3.859476078 \\
\hline $\mathrm{H}$ & 1.497639504 & 1.366743857 & -4.441064776 \\
\hline $\mathrm{H}$ & 2.509178160 & 1.319611800 & -3.262966855 \\
\hline $\mathrm{O}$ & -3.378610272 & 0.383589278 & -5.914241586 \\
\hline $\mathrm{H}$ & -2.440188934 & 0.456215764 & -6.147770934 \\
\hline $\mathrm{H}$ & -3.651299316 & -0.466369476 & -6.283854092 \\
\hline \multicolumn{4}{|c|}{$\left[\mathrm{PdCl}_{4}\right]^{2-}$} \\
\hline & -1.644781223 & 1.673043290 & 0.075446341 \\
\hline
\end{tabular}


$\begin{array}{llll}\mathrm{Cl} & -1.684948635 & -1.634286640 & -0.074755885\end{array}$

$\begin{array}{llll}\mathrm{Cl} & 1.638077922 & -1.663711955 & 0.074482526\end{array}$

$\mathrm{Cl} \quad 1.642644248 \quad 1.653651696 \quad-0.074825176$

$\begin{array}{llll}\text { Pd } & -0.010803987 & 0.001209447 & -0.00034780\end{array}$

$\left[\mathrm{PdCl}_{4}\right]^{2-} \mathrm{SMD}$

$\begin{array}{rrrr}\mathrm{Cl} & -1.644781223 & 1.673043290 & 0.075446341 \\ \mathrm{Cl} & -1.684948635 & -1.634286640 & -0.074755885 \\ \mathrm{Cl} & 1.638077922 & -1.663711955 & 0.074482526 \\ \mathrm{Cl} & 1.642644248 & 1.653651696 & -0.074825176 \\ \mathrm{Pd} & -0.010803987 & 0.001209447 & -0.000347806\end{array}$

